Article

\title{
New Scientific Contribution on the 2-D Subdomain Technique in Polar Coordinates: Taking into account of Iron Parts
}

\author{
Frédéric Dubas ${ }^{1, *}$ and Kamel Boughrara ${ }^{2}$ \\ 1 Département ENERGIE, FEMTO-ST, CNRS, Univ. Bourgogne Franche-Comté, F90000 Belfort, France \\ 2 Laboratoire de Rcherche en Electrotechnique (LRE-ENP), Algiers, 10 av. Pasteur, El Harrach, BP 182, 16200, \\ Algeria; kamel.boughrara@g.enp.edu.dz \\ * Correspondence: frederic.dubas@univ-fcomte.fr; Tel.: +33-38-457-8203
}

\begin{abstract}
This paper presents a new scientific contribution on the two-dimensional (2-D) subdomain technique in polar coordinates taking into account the finite relative permeability of the ferromagnetic material. The constant relative permeability corresponds to linear part of the nonlinear $B(H)$ curve. As in conventional technique, the method of separation of variables and the Fourier's series are used for the resolution of magnetostatic Maxwell's equations in each region. Although, the general solutions of magnetic field in the subdomains and boundary conditions (BCs) between regions are different in the conventional and proposed method. In this later, the magnetic field solution in each subdomain is a superposition of two magnetic quantities in the two directions (i.e., $r$ - and $\Theta$-axis) and the $\mathrm{BCs}$ between two regions are also in both directions. For example, the scientific contribution has been applied to an air- or iron-cored coil supplied by a constant current. The distribution of local quantities (i.e., the magnetic vector potential and flux density) has been validated by a corresponding 2-D finite-element analysis (FEA). The obtained semi-analytical results are in very good agreement with those of numerical method.
\end{abstract}

Keywords: air- or iron-cored coil; polar coordinates; fourier analysis; two-dimensional; subdomain technique

\section{Introduction}

The full calculation of magnetic field in electrical engineering applications is the first step for their design and optimization, the methods of magnetic field prediction can be classified in various categories [1]:

- Lehmann's graphical [2];

- Numerical (i.e., finite-element, finite-difference, boundary-element, etc.) [3-5];

- Equivalent circuit (i.e., electrical, thermal, magnetic, etc.) [6-8];

- Schwarz-Christoffel mapping (i.e., conformal transformation, complex permeance model, etc.) [9];

- Maxwell-Fourier [10-15].

Currently, the works of design are based on (semi-)analytical models ${ }^{1}$ (i.e., equivalent circuit, conformal transformation and Maxwell-Fourier methods). In comparison with the other methods, 
under certain geometrical and physical assumptions, these models permit to obtain accurate analytical expressions of magnetic field and known as fast for the prediction of local and global electromagnetic performances. At present, Maxwell-Fourier methods are one of the most used semi-analytic approaches with very accurate results (i.e., error less than $5 \%$ ) on the electromagnetic performances calculation. These models are based on the formal resolution of Maxwell's equations in Cartesian, cylindrical or spherical coordinates by using the method of seperation of variables and the Fourier's series. Taking into account of iron parts and/or the effect of local/global saturation is still a scientific challenge in Maxwell-Fourier methods which is rarely explored in the literature [17,18]. Recently, Dubas et al. (2017) [1] realized an overview on the existing (semi-)analytical models in Maxwell-Fourier methods with the effect of local/global saturation, which can thus be classified as follows

- Multi-layers models (i.e., Carter's coefficient [19,20], saturation coefficient [21,22], concept wave impedance [23-26], and convolution theorem [27-30]);

- $\quad$ Eigenvalues model, viz., the method of Truncation Region Eigenfunction Expansions (TREE) [31,32];

- $\quad$ Subdomain technique $[1,33,34]$;

- Hybrid models, viz., the analytical solution combined with numerical methods $[35,36]$ or (non)linear magnetic equivalent circuit [37-39].

The consideration of the effect of local/global saturation is appearing in hybrid models, where the solution is established analytically in concentric regions of very low permeability (e.g., air-gap and magnets) and other methods (e.g., numerical or magnetic equivalent circuit) are sought in regions where the saturation effect cannot be neglected. On other hand, the other models (i.e., multi-layers models, TREE method and sudomain technique) are more focused the global saturation. Some details and (dis)advantages of these techniques can be found in [1]. In most semi-analytical models based on the subdomain technique, the iron parts are considered to be infinitely permeability due the variation of material proprieties in the various directions, so that the saturation effect is neglected [16-18]. The first paper introducing the iron parts in the magnetic field calculation by using subdomain technique is [1], where the authors solve partial differential equations (EDPs) of magnetic potential vector in Cartesian coordinates in which the subdomains connection is performed directly in both directions (i.e., $x$ - and $y$-edges). The 2-D magnetostatic model has been applied to an air- or iron-cored coil supplied by a constant current. In [33], the authors propose a 2-D semi-analytical model in spoke-type magnets synchronous machines based on the subdomain technique in polar coordinates with Taylor polynomial of degree 3 by focusing on the consideration of iron. The iron magnetic permeability is supposed constant corresponding to linear zone of the nonlinear $B(H)$ curve. The subdomains connection is carried out in both directions (i.e., $r$ - and $\Theta$-edges). The general solution of magnetic field is obtained by using the traditional BCs, in addition to new radial BCs (e.g., between the magnets and the rotor teeth, between the teeth and the slots of the stator) which are traduced into a system of linear equations according to Taylor series expansion. In [34], this semi-analytical model has been extended taking into account the initial magnetization curve in each soft-magnetic subdomain by an iterative procedure.

In the literature, to the authors' knowledge, there exists no exact 2-D subdomain technique in polar coordinates taking into account of iron parts with(out) the nonlinear $B(H)$ curve and not using the Taylor series expansion to satisfy the $r$-edges BCs. Thus, the research work in this paper contributes to the continuous improvement of the 2-D subdomain technique. Moreover, it is an extension of [1] in polar coordinates $(r, \Theta)$. Section 2 presents this new scientific contribution. By applying the principle of superposition on the magnetic quantities in order to respect the BCs on the various edges, the general solution of magnetic field is decomposed in Fourier's series into two general solutions in both directions (i.e., $r$ - and $\Theta$-edges). It allows evaluation of the local distribution of flux densities in the iron parts with a global saturation, does not have numerical convergence problems contrary to others models, and would easily introduce the current penetration effect in the conductive materials. The semi-analytical solution is exact as in [1] and does not use the Taylor polynomial to satisfy the $r$-edges BCs contrary to $[33,34]$. For example, it was applied to an air- or iron-cored coil supplied by a constant 


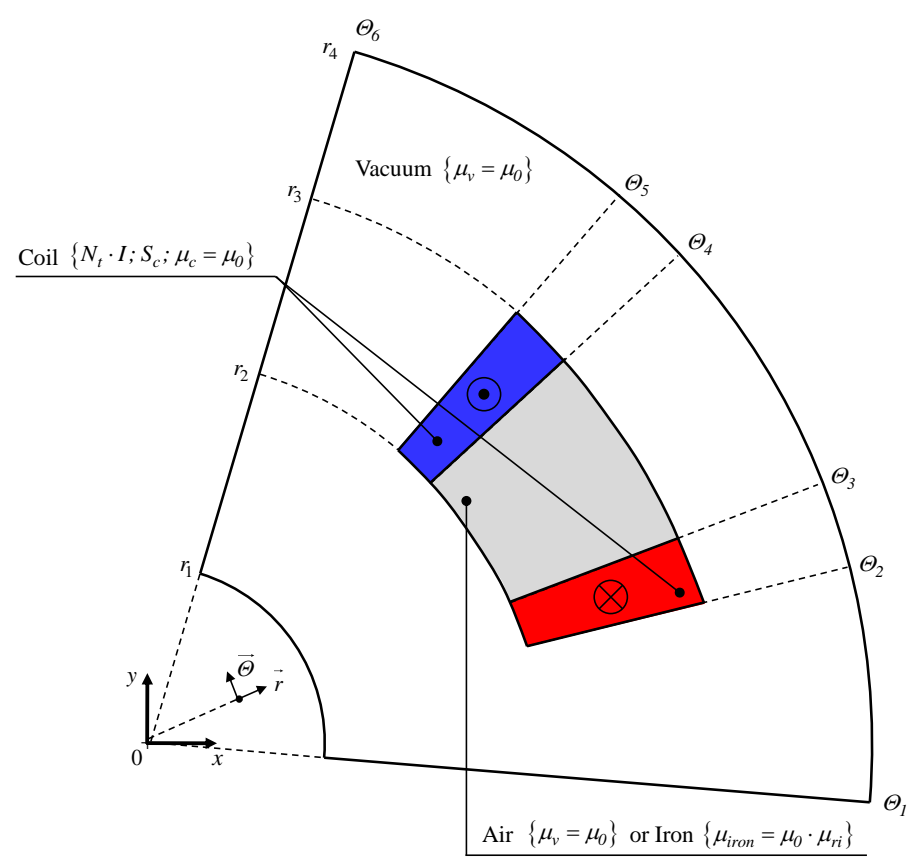

Figure 1. Physical and geometrical parameters (see Table 1) of air- or iron-cored coil where $\otimes$ and $\odot$ are respectively the forward and return conductor.

current. The iron magnetic permeability is constant corresponding to linear zone of the nonlinear $B(H)$ curve $[1,33]$. Nevertheless, as in $[29,30,34]$, the saturation effect could be taken into account by an iterative calculation considering, at each iteration, a constant relative magnetic permeability according to the nonlinear $B(H)$ curve. However, this is beyond the scope of the paper. In Section 3, in order to confirm the effectiveness of the proposed technique, all semi-analytical results are then compared to those found by 2-D FEA [40]. The comparisons are very satisfying in amplitudes and waveforms.

\section{A 2-D Subdomain Technique of Magnetic Field in Polar Coordinates}

\subsection{Model Description and Assumptions}

Figure 1 represents the physical and geometrical parameters of an air- or iron-cored coil with $N_{t}$ turns of copper wire supplied by a constant current $I$. The electromagnetic device is surrounded by an infinite box with null value of magnetic vector potential at it boundaries.

The analytical prediction of magnetic field based on the 2-D subdomain technique is done by solving magnetostatic Maxwell's equations in polar coordinates $(r, \Theta)$ with the following assumptions:

- The magnetic vector potential has only one component along the $z$-axis (i.e., $\mathbf{A}=\left\{0 ; 0 ; A_{z}\right\}$ ) and then the end-effects are not considered;

- All materials are isotropic and the permeabilities are supposed constants in both directions (i.e., $r$ and $\Theta$-axis);

- All electrical conductivities of materials are supposed nulls (i.e., the eddy-currents induced in the copper/iron are neglected).

\subsection{Problem Discretization in Regions}

In Figure 2, we present the studied electromagnetic device which is divided into 7 regions with $\mu=C^{s t}$, viz.,

- Region $1\left\{\forall \Theta \wedge r \in\left[r_{1}, r_{2}\right]\right\}$ with $\mu_{1}=\mu_{v}$;

- Region $2\left\{\forall \Theta \wedge r \in\left[r_{3}, r_{4}\right]\right\}$ with $\mu_{2}=\mu_{v}$; 


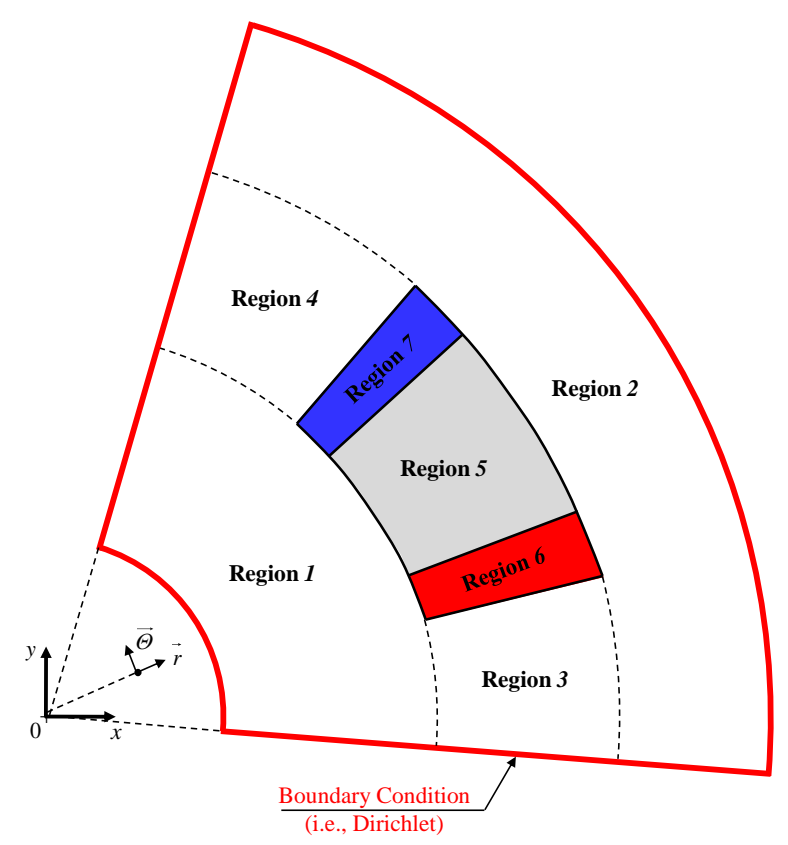

Figure 2. Definition of regions in the air- or iron-cored coil.

- Region $3\left\{\Theta \in\left[\Theta_{1}, \Theta_{2}\right] \wedge r \in\left[r_{2}, r_{3}\right]\right\}$ with $\mu_{3}=\mu_{v}$;

- Region $4\left\{\Theta \in\left[\Theta_{5}, \Theta_{6}\right] \wedge r \in\left[r_{2}, r_{3}\right]\right\}$ with $\mu_{4}=\mu_{v}$;

- Region 5 (i.e., the air or iron in the middle of the coil) $\left\{\Theta \in\left[\Theta_{2}, \Theta_{3}\right] \wedge r \in\left[r_{2}, r_{3}\right]\right\}$ with $\mu_{5}=\mu_{v}$ for the air or $\mu_{5}=\mu_{\text {iron }}$ for the iron;

- Region 6 (i.e., the forward conductor) $\left\{\Theta \in\left[\Theta_{2}, \Theta_{3}\right] \wedge r \in\left[r_{2}, r_{3}\right]\right\}$ with $\mu_{6}=\mu_{c}$;

- Region 7 (i.e., the return conductor) $\left\{\Theta \in\left[\Theta_{4}, \Theta_{5}\right] \wedge r \in\left[r_{2}, r_{3}\right]\right\}$ with $\mu_{7}=\mu_{c}$.

\subsection{Governing EDPs in Polar Coordinates: Laplace's and Poisson's Equations}

According to (A.1) (see Appendix A), the distribution of magnetic vector potential in polar coordinates $(r, \Theta)$ is governed by

$$
\begin{gathered}
\Delta A_{z j}=\frac{\partial^{2} A_{z j}}{\partial r^{2}}+\frac{1}{r} \cdot \frac{\partial A_{z j}}{\partial r}+\frac{1}{r^{2}} \cdot \frac{\partial^{2} A_{z j}}{\partial \Theta^{2}}=0 \quad \text { for } \quad j=\{1, \ldots, 5\} \quad \text { (Laplace's equation), } \\
\Delta A_{z k}=\frac{\partial^{2} A_{z k}}{\partial r^{2}}+\frac{1}{r} \cdot \frac{\partial A_{z k}}{\partial r}+\frac{1}{r^{2}} \cdot \frac{\partial^{2} A_{z k}}{\partial \Theta^{2}}=-\mu_{k} \cdot J_{z k} \quad \text { for } k=\{6,7\} \quad \text { (Poisson's equation), }
\end{gathered}
$$

where $J_{z k}$ is the current density of the coil defined by

$$
J_{z k}=C_{k} \cdot \frac{N_{t} \cdot I}{S_{c}},
$$

in which $S_{c}$ is the conductor surface, and $C_{k}$ (with $C_{6}=1$ and $C_{7}=-1$ ) is the coefficient that represents the current direction in the conductor.

According to Appendix A, the resolution of Laplace's and Poisson's equations by using the method of separation of variables and the Fourier's series permit to obtain two potentials in both directions, viz., $A_{z \bullet}^{\Theta}$ for the $\Theta$-edges (A.2b) and $A_{z \bullet}^{r}$ for the $r$-edges (A.2c). The spatial frequency (or periodicity) of $A_{z \bullet}^{\Theta}$ and $A_{z \bullet}^{r}$ are respectively defined by $\beta_{\bullet_{\bullet}}$ and $\lambda_{\bullet} \bullet$ with $h \bullet$ and $n \bullet$ the spatial harmonic orders. 


\subsection{Definition of $B C s$}

In electromagnetic, the general solutions of various regions depend on the BCs at the interface of two surfaces, which are defined by the continuity of the normal flux density $\mathbf{B}_{\perp}$ and parallel field intensity $\mathbf{H}_{\|}$[1]. On the outer BCs for $\left(\Theta_{1} \wedge \Theta_{6}, \forall r\right)$ and $\left(\forall \Theta, r_{1} \wedge r_{4}\right), A_{z}$ satisfies the Dirichlet BC (see Figure 2), viz., $A_{z}=0$.

Figure 3 represents the respective $\mathrm{BCs}$ at the interface between the various regions in both directions (i.e., $r$ - and $\Theta$-edges).

\subsection{General Solutions of Various Regions}

\subsubsection{Region 1}

The solution of $A_{z 1}, B_{r 1}$ and $B_{\Theta 1}$ are determined by the case-study no 1 (i.e., $A_{z}$ imposed on all edges of a region) in Appendix B. The BCs on the $r$-edges of the region (see Figure $3 a$ ) are met by posing $c_{h}^{\Theta}=0$ in (B.6). Therefore, $A_{z 1}$ satisfying the BCs of Figure 3 a and solution of (1a) is given by

$$
A_{z 1}=-\sum_{h 1=1}^{\infty} d 1_{h 1}^{\Theta} \cdot \frac{r_{2}}{\beta 1_{h 1}} \cdot \frac{E_{\phi}\left(\beta 1_{h 1}, r, r_{1}\right)}{P_{\not}\left(\beta 1_{h 1}, r_{2}, r_{1}\right)} \cdot \sin \left[\beta 1_{h 1} \cdot\left(\Theta-\Theta_{1}\right)\right]
$$

the components of $\mathbf{B}_{\mathbf{1}}=\left\{B_{r 1} ; B_{\Theta 1} ; 0\right\}$ by

$$
\begin{gathered}
B_{r 1}=-\sum_{h 1=1}^{\infty} d 1_{h 1}^{\Theta} \cdot \frac{r_{2}}{r} \cdot \frac{E_{\phi}\left(\beta 1_{h 1}, r, r_{1}\right)}{P_{\phi}\left(\beta 1_{h 1}, r_{2}, r_{1}\right)} \cdot \cos \left[\beta 1_{h 1} \cdot\left(\Theta-\Theta_{1}\right)\right], \\
B_{\Theta 1}=\sum_{h 1=1}^{\infty} d 1_{h 1}^{\Theta} \cdot \frac{r_{2}}{r} \cdot \frac{P_{\phi}\left(\beta 1_{h 1}, r, r_{1}\right)}{P_{\phi}\left(\beta 1_{h 1}, r_{2}, r_{1}\right)} \cdot \sin \left[\beta 1_{h 1} \cdot\left(\Theta-\Theta_{1}\right)\right],
\end{gathered}
$$

where $E_{\phi}(w, x, y)$ and $P_{\phi}(w, x, y)$ are defined in (B.4), $h 1$ the spatial harmonic orders in Region $1, d 1_{h 1}^{\Theta}$ the integration constant, and $\beta 1_{h 1}=h 1 \cdot \pi / \tau_{\Theta 1}$ with $\tau_{\Theta 1}=\Theta_{6}-\Theta_{1}$.

Using a Fourier series expansion of $F_{1}(\Theta)$ (see Figure 3a) over the interval $\Theta=\left[\Theta_{1}, \Theta_{6}\right]=$ $\left[\Theta_{1}, \Theta_{1}+\tau_{\Theta 1}\right]$, the integration constant $d 1_{h 1}^{\Theta}$ is determined in Appendix $C$ with

$$
d 1_{h 1}^{\Theta}=\frac{2}{\tau_{\Theta 1}} \cdot \int_{\Theta_{1}}^{\Theta_{1}+\tau_{\Theta 1}} F_{1}(\Theta) \cdot \sin \left[\beta 1_{h 1} \cdot\left(\Theta-\Theta_{1}\right)\right] \cdot d \Theta .
$$

\subsubsection{Region 2}

The same method than Region 1 is used to define the general solution in Region 2. By posing $d_{h}^{\Theta}=0$ in (B.6) (see Appendix B), $A_{z 2}$ satisfying the BCs of Figure $3 \mathrm{~b}$ and solution of (1a) is given by

$$
A_{z 2}=\sum_{h 2=1}^{\infty} c 2_{h 2}^{\Theta} \cdot \frac{r_{3}}{\beta 2_{h 2}} \cdot \frac{E_{\phi}\left(\beta 2_{h 2}, r_{4}, r\right)}{P_{\phi}\left(\beta 2_{h 2}, r_{4}, r_{3}\right)} \cdot \sin \left[\beta 2_{h 2} \cdot\left(\Theta-\Theta_{1}\right)\right]
$$

the components of $\mathbf{B}_{\mathbf{2}}=\left\{B_{r 2} ; B_{\Theta 2} ; 0\right\}$ by

$$
\begin{aligned}
& B_{r 2}=\sum_{h 2=1}^{\infty} c 2_{h 2}^{\Theta} \cdot \frac{r_{3}}{r} \cdot \frac{E_{\not \phi}\left(\beta 2_{h 2}, r_{4}, r\right)}{P_{\phi}\left(\beta 2_{h 2}, r_{4}, r_{3}\right)} \cdot \cos \left[\beta 2_{h 2} \cdot\left(\Theta-\Theta_{1}\right)\right], \\
& B_{\Theta 2}=\sum_{h 2=1}^{\infty} c 2_{h 2}^{\Theta} \cdot \frac{r_{3}}{r} \cdot \frac{P_{\phi}\left(\beta 2_{h 2}, r_{4}, r\right)}{P_{\phi}\left(\beta 2_{h 2}, r_{4}, r_{3}\right)} \cdot \sin \left[\beta 2_{h 2} \cdot\left(\Theta-\Theta_{1}\right)\right],
\end{aligned}
$$


where $h 2$ is the spatial harmonic orders in Region $2, c 2_{h 2}^{\Theta}$ the integration constant, and $\beta 2_{h 2}=h 2 \cdot \pi / \tau_{\Theta 2}$ with $\tau_{\Theta 2}=\Theta_{6}-\Theta_{1}$.

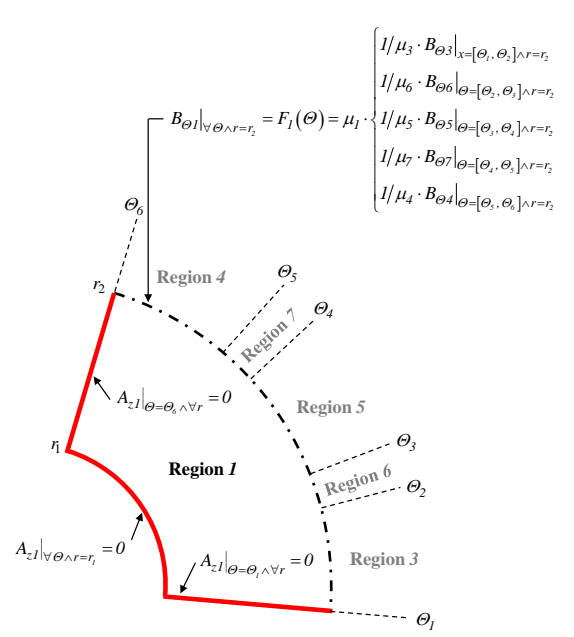

(a)

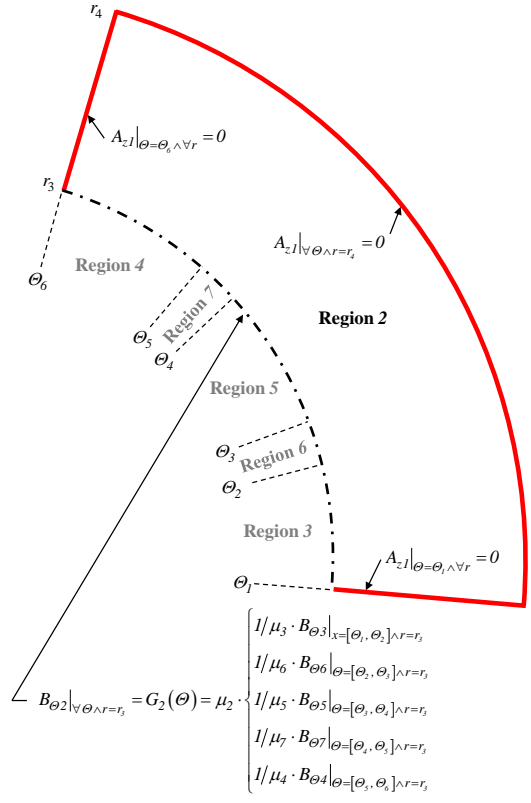

(b)

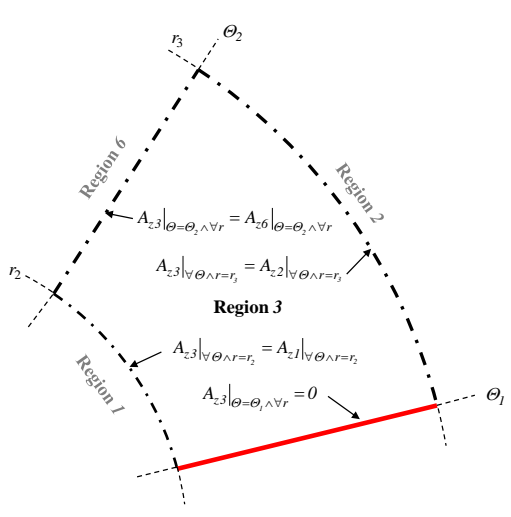

(c)

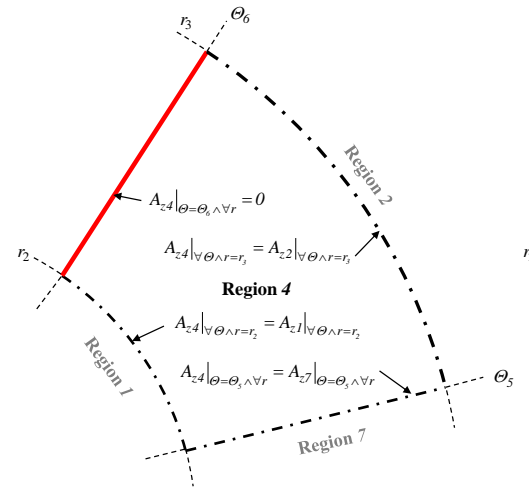

(d)

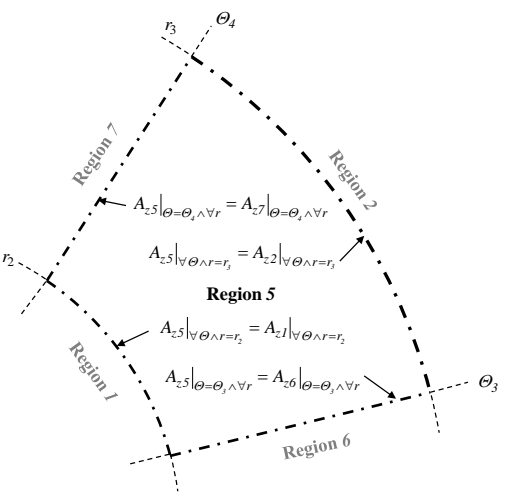

(e)

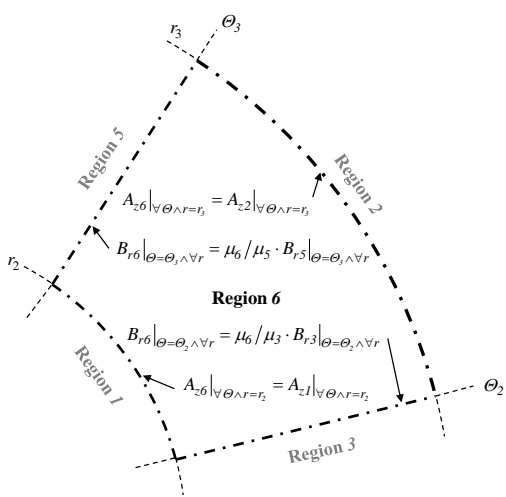

(f)

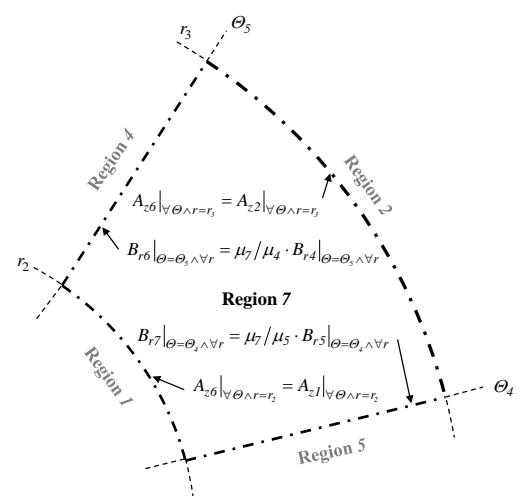

(g)

Figure 3. Boundary conditions (BCs) in both directions (i.e., $r$ - and $\Theta$-edges): (a) Region 1, (b) Region 2 , (c) Region 3, (d) Region 4, (e) Region 5, (f) Region 6, and (g) Region 7. 
Using a Fourier series expansion of $G_{2}(\Theta)$ (see Figure 3b) over the interval $\Theta=\left[\Theta_{1}, \Theta_{6}\right]=$ $\left[\Theta_{1}, \Theta_{1}+\tau_{\Theta 2}\right]$, the integration constant $c 2_{h 2}^{\Theta}$ is determined in Appendix $C$ with

$$
c 2_{h 2}^{\Theta}=\frac{2}{\tau_{\Theta 2}} \cdot \int_{\Theta_{1}}^{\Theta_{1}+\tau_{\Theta 2}} G_{2}(\Theta) \cdot \sin \left[\beta 2_{h 2} \cdot\left(\Theta-\Theta_{1}\right)\right] \cdot d \Theta .
$$

\subsubsection{Region 3}

The solution of $A_{z 3}, B_{r 3}$ and $B_{\Theta 3}$ are determined by the case-study no 1 (i.e., $A_{z}$ imposed on all edges of a region) in Appendix $B$. The $B C$ s on the $\Theta$-edges of the region (see Figure 3c) are met by posing $e_{n}^{r}=0$ in (B.1) - (B.3). Therefore, $A_{z 3}$ satisfying the BCs of Figure $3 \mathrm{c}$ and solution of (1a) is given by

$$
\begin{gathered}
A_{z 3}=A_{z 3}^{\Theta}+A_{z 3}^{r} \\
A_{z 3}^{\Theta}=\sum_{h 3=1}^{\infty}\left[c 3_{h 3}^{\Theta} \cdot r_{2} \cdot \frac{E_{\phi}\left(\beta 3_{h 3}, r_{3}, r\right)}{E_{\phi}\left(\beta 3_{h 3}, r_{3}, r_{2}\right)}+d 3_{h 3}^{\Theta} \cdot r_{3} \cdot \frac{E_{\phi}\left(\beta 3_{h 3}, r, r_{2}\right)}{E_{\phi}\left(\beta 3_{h 3}, r_{3}, r_{2}\right)}\right] \cdot \sin \left[\beta 3_{h 3} \cdot\left(\Theta-\Theta_{1}\right)\right], \\
A_{z 3}^{r}=\sum_{n 3=1}^{\infty} f 3_{n 3}^{r} \cdot r_{2} \cdot \frac{\operatorname{sh}\left[\lambda 3_{n 3} \cdot\left(\Theta-\Theta_{1}\right)\right]}{\operatorname{sh}\left(\lambda 3_{n 3} \cdot \tau_{\Theta 3}\right)} \cdot \sin \left[\lambda 3_{n 3} \cdot \ln \left(\frac{r}{r_{2}}\right)\right],
\end{gathered}
$$

the $r$-component of $\mathbf{B}_{3}$ by

$$
\begin{gathered}
B_{r 3}=B_{r 3}^{\Theta}+B_{r 3}^{r}, \\
B_{r 3}^{\Theta}=\sum_{h 3=1}^{\infty} \beta 3_{h 3} \cdot\left[c 3_{h 3}^{\Theta} \cdot \frac{r_{2}}{r} \cdot \frac{E_{q}\left(\beta 3_{h 3}, r_{3}, r\right)}{E_{\phi}\left(\beta 3_{h 3}, r_{3}, r_{2}\right)}+d 3_{h 3}^{\Theta} \cdot \frac{r_{3}}{r} \cdot \frac{E_{q}\left(\beta 3_{h 3}, r, r_{2}\right)}{E_{\phi}\left(\beta 3_{h 3}, r_{3}, r_{2}\right)}\right] \cdot \cos \left[\beta 3_{h 3} \cdot\left(\Theta-\Theta_{1}\right)\right], \\
B_{r 3}^{r}=\sum_{n 3=1}^{\infty} \lambda 3_{n 3} \cdot f 3_{n 3}^{r} \cdot \frac{r_{2}}{r} \cdot \frac{c h\left[\lambda 3_{n 3} \cdot\left(\Theta-\Theta_{1}\right)\right]}{\operatorname{sh}\left(\lambda 3_{n 3} \cdot \tau_{\Theta 3}\right)} \cdot \sin \left[\lambda 3_{n 3} \cdot \ln \left(\frac{r}{r_{2}}\right)\right],
\end{gathered}
$$

the $\Theta$-component of $\mathbf{B}_{3}$ by

$$
\begin{gathered}
B_{\Theta 3}=B_{\Theta 3}^{\Theta}+B_{\Theta 3}^{r}, \\
B_{\Theta 3}^{\Theta}=\sum_{h 3=1}^{\infty} \beta 3_{h 3} \cdot\left[c 3_{h 3}^{\Theta} \cdot \frac{r_{2}}{r} \cdot \frac{P_{\phi}\left(\beta 3_{h 3}, r_{3}, r\right)}{E_{\phi}\left(\beta 3_{h 3}, r_{3}, r_{2}\right)}-d 3_{h 3}^{\Theta} \cdot \frac{r_{3}}{r} \cdot \frac{P_{\phi}\left(\beta 3_{h 3}, r, r_{2}\right)}{E_{\phi}\left(\beta 3_{h 3}, r_{3}, r_{2}\right)}\right] \cdot \sin \left[\beta 3_{h 3} \cdot\left(\Theta-\Theta_{1}\right)\right], \\
B_{\Theta 3}^{r}=-\sum_{n 3=1}^{\infty} \lambda 3_{n 3} \cdot f 3_{n 3}^{r} \cdot \frac{r_{2}}{r} \cdot \frac{\operatorname{sh}\left[\lambda 3_{n 3} \cdot\left(\Theta-\Theta_{1}\right)\right]}{\operatorname{sh}\left(\lambda 3_{n 3} \cdot \tau_{\Theta 3}\right)} \cdot \cos \left[\lambda 3_{n 3} \cdot \ln \left(\frac{r}{r_{2}}\right)\right],
\end{gathered}
$$

where $h 3$ and $n 3$ are the spatial harmonic orders in Region $3 ; c 3_{h 3}^{\Theta}, d 3_{h 3}^{\Theta}$ and $f 3_{n 3}^{r}$ the integration constants; $\beta 3_{h 3}=h 3 \cdot \pi / \tau_{\Theta 3}$ with $\tau_{\Theta 3}=\Theta_{2}-\Theta_{1}$; and $\lambda 3_{n 3}=n 3 \cdot \pi / \tau_{r 3}$ with $\tau_{r 3}=\ln \left(r_{3} / r_{2}\right)$.

Using Fourier series expansion of $\left.A_{z 1}\right|_{\forall \Theta \wedge r=r_{2}}$ and $\left.A_{z 2}\right|_{\forall \Theta \wedge r=r_{3}}$ (see Figure 3c) over the interval $\Theta=\left[\Theta_{1}, \Theta_{2}\right]=\left[\Theta_{1}, \Theta_{1}+\tau_{\Theta 3}\right]$, the integration constants $c 3_{h 3}^{\Theta}$ and $d 3_{h 3}^{\Theta}$ are determined in Appendix $C$ with

$$
\begin{aligned}
& c 3_{h 3}^{\Theta}=\frac{2}{\tau_{\Theta 3}} \cdot \int_{\Theta_{1}}^{\Theta_{1}+\tau_{\Theta 3}} \frac{\left.A_{z 1}\right|_{r=r_{2}}}{r_{2}} \cdot \sin \left[\beta 3_{h 3} \cdot\left(\Theta-\Theta_{1}\right)\right] \cdot d \Theta, \\
& d 3_{h 3}^{\Theta}=\frac{2}{\tau_{\Theta 3}} \cdot \int_{\Theta_{1}}^{\Theta_{1}+\tau_{\Theta 3}} \frac{\left.A_{z 2}\right|_{r=r_{3}}}{r_{3}} \cdot \sin \left[\beta 3_{h 3} \cdot\left(\Theta-\Theta_{1}\right)\right] \cdot d \Theta .
\end{aligned}
$$

With a weighting function $g(r)=r^{-1}$ and using a Fourier series expansion of $\left.A_{z 6}\right|_{\Theta=\Theta_{2} \wedge \forall r}$ (see Figure 3c) over the interval $r=\left[r_{2}, r_{3}\right]$, the integration constant $f 3_{n 3}^{r}$ is determined in Appendix $C$ with

$$
f 3_{n 3}^{r}=\frac{2}{\tau_{r 3}} \cdot \int_{r_{2}}^{r_{3}} \frac{1}{r} \cdot \frac{\left.A_{z 6}\right|_{\Theta=\Theta_{2}}}{r_{2}} \cdot \sin \left[\lambda 3_{n 3} \cdot \ln \left(\frac{r}{r_{2}}\right)\right] \cdot d r
$$




\subsubsection{Region 4}

The solution in Region 4 is obtained using the same development than Region 3. By posing $f_{n}^{r}=0$ in (B.1) - (B.3) (see Appendix B), $A_{z 4}$ satisfying the BCs of Figure $3 \mathrm{~d}$ and solution of (1a) is given by

$$
\begin{gathered}
A_{z 4}=A_{z 4}^{\Theta}+A_{z 4}^{r} \\
A_{z 4}^{\Theta}=\sum_{h 4=1}^{\infty}\left[c 4_{h 4}^{\Theta} \cdot r_{2} \cdot \frac{E_{\phi}\left(\beta 4_{h 4}, r_{3}, r\right)}{E_{\phi}\left(\beta 4_{h 4}, r_{3}, r_{2}\right)}+d 4_{h 4}^{\Theta} \cdot r_{3} \cdot \frac{E_{\phi}\left(\beta 4_{h 4}, r_{,} r_{2}\right)}{E_{\phi}\left(\beta 4_{h 4}, r_{3}, r_{2}\right)}\right] \cdot \sin \left[\beta 4_{h 4} \cdot\left(\Theta-\Theta_{5}\right)\right], \\
A_{z 4}^{r}=\sum_{n 4=1}^{\infty} e 4_{n 4}^{r} \cdot r_{2} \cdot \frac{\operatorname{sh}\left[\lambda 4_{n 4} \cdot\left(\Theta_{6}-\Theta\right)\right]}{\operatorname{sh}\left(\lambda 4_{n 4} \cdot \tau_{\Theta 4}\right)} \cdot \sin \left[\lambda 4_{n 4} \cdot \ln \left(\frac{r}{r_{2}}\right)\right],
\end{gathered}
$$

the $r$-component of $\mathbf{B}_{4}$ by

$$
\begin{gathered}
B_{r 4}=B_{r 4}^{\Theta}+B_{r 4}^{r}, \\
B_{r 4}^{\Theta}=\sum_{h 4=1}^{\infty} \beta 4_{h 4} \cdot\left[c 4_{h 4}^{\Theta} \cdot \frac{r_{2}}{r} \cdot \frac{E_{q}\left(\beta 4_{h 4}, r_{3}, r\right)}{E_{\phi}\left(\beta 4_{h 4}, r_{3}, r_{2}\right)}+d 4_{h 4}^{\Theta} \cdot \frac{r_{3}}{r} \cdot \frac{E_{q}\left(\beta 4_{h 4}, r, r_{2}\right)}{E_{\phi}\left(\beta 4_{h 4}, r_{3}, r_{2}\right)}\right] \cdot \cos \left[\beta 4_{h 4} \cdot\left(\Theta-\Theta_{5}\right)\right], \\
B_{r 4}^{r}=-\sum_{n 4=1}^{\infty} \lambda 4_{n 4} \cdot e 4_{n 4}^{r} \cdot \frac{r_{2}}{r} \cdot \frac{c h\left[\lambda 4_{n 4} \cdot\left(\Theta_{6}-\Theta\right)\right]}{\operatorname{sh}\left(\lambda 4_{n 4} \cdot \tau_{\Theta 4}\right)} \cdot \sin \left[\lambda 4_{n 4} \cdot \ln \left(\frac{r}{r_{2}}\right)\right],
\end{gathered}
$$

the $\Theta$-component of $\mathbf{B}_{4}$ by

$$
\begin{gathered}
B_{\Theta 4}=B_{\Theta 4}^{\Theta}+B_{\Theta 4}^{r} \\
B_{\Theta 4}^{\Theta}=\sum_{h 4=1}^{\infty} \beta 4_{h 4} \cdot\left[c 4_{h 4}^{\Theta} \cdot \frac{r_{2}}{r} \cdot \frac{P_{\phi}\left(\beta 4_{h 4}, r_{3}, r\right)}{E_{\phi}\left(\beta 4_{h 4}, r_{3}, r_{2}\right)}-d 4_{h 4}^{\Theta} \cdot \frac{r_{3}}{r} \cdot \frac{P_{\phi}\left(\beta 4_{h 4}, r, r_{2}\right)}{E_{\phi}\left(\beta 4_{h 4}, r_{3}, r_{2}\right)}\right] \cdot \sin \left[\beta 4_{h 4} \cdot\left(\Theta-\Theta_{5}\right)\right], \\
B_{\Theta}^{r}=-\sum_{n 4=1}^{\infty} \lambda 4_{n 4} \cdot e 4_{n 4}^{r} \cdot \frac{r_{2}}{r} \cdot \frac{s h\left[\lambda 4_{n 4} \cdot\left(\Theta_{6}-\Theta\right)\right]}{s h\left(\lambda 4_{n 4} \cdot \tau_{\Theta 4}\right)} \cdot \cos \left[\lambda 4_{n 4} \cdot \ln \left(\frac{r}{r_{2}}\right)\right],
\end{gathered}
$$

where $h 4$ and $n 4$ are the spatial harmonic orders in Region $4 ; c 4_{h 4}^{\Theta}, d 4_{h 4}^{\Theta}$ and $e 4_{n 4}^{r}$ the integration constants; $\beta 4_{h 4}=h 4 \cdot \pi / \tau_{\Theta 4}$ with $\tau_{\Theta 4}=\Theta_{6}-\Theta_{5} ;$ and $\lambda 4_{n 4}=n 4 \cdot \pi / \tau_{r 4}$ with $\tau_{r 4}=\ln \left(r_{3} / r_{2}\right)$.

Using Fourier series expansion of $\left.A_{z 1}\right|_{\forall \Theta \wedge r=r_{2}}$ and $\left.A_{z 2}\right|_{\forall \Theta \wedge r=r_{3}}$ (see Figure 3d) over the interval $\Theta=\left[\Theta_{5}, \Theta_{6}\right]=\left[\Theta_{5}, \Theta_{5}+\tau_{\Theta 4}\right]$, the integration constants $c 4_{h 4}^{\Theta}$ and $d 4_{h 4}^{\Theta}$ are determined in Appendix $C$ with

$$
\begin{aligned}
& c 4_{h 4}^{\Theta}=\frac{2}{\tau_{\Theta 4}} \cdot \int_{\Theta_{5}}^{\Theta_{5}+\tau_{\Theta 4}} \frac{\left.A_{z 1}\right|_{r=r_{2}}}{r_{2}} \cdot \sin \left[\beta 4_{h 4} \cdot\left(\Theta-\Theta_{5}\right)\right] \cdot d \Theta, \\
& d 4_{h 4}^{\Theta}=\frac{2}{\tau_{\Theta 4}} \cdot \int_{\Theta_{5}}^{\Theta_{5}+\tau_{\Theta 4}} \frac{\left.A_{z 2}\right|_{r=r_{3}}}{r_{3}} \cdot \sin \left[\beta 4_{h 4} \cdot\left(\Theta-\Theta_{5}\right)\right] \cdot d \Theta .
\end{aligned}
$$

With a weighting function $g(r)=r^{-1}$ and using a Fourier series expansion of $\left.A_{z 7}\right|_{\Theta=\Theta_{5} \wedge \forall r}$ (see Figure 3d) over the interval $r=\left[r_{2}, r_{3}\right]$, the integration constant $e 4_{n 4}^{r}$ is determined in Appendix C with

$$
e 4_{n 4}^{r}=\frac{2}{\tau_{r 4}} \cdot \int_{r_{2}}^{r_{3}} \frac{1}{r} \cdot \frac{\left.A_{z 7}\right|_{\Theta=\Theta_{5}}}{r_{2}} \cdot \sin \left[\lambda 4_{n 4} \cdot \ln \left(\frac{r}{r_{2}}\right)\right] \cdot d r
$$

\subsubsection{Region 5}

For Region 5, the general solution is given according to the BCs of case-study no 1 (i.e., $A_{z}$ imposed on all edges of a region) in Appendix B. Therefore, $A_{z 5}$ satisfying the BCs of Figure $3 \mathrm{e}$ and solution of (1a) is given by

$$
A_{z 5}=A_{z 5}^{\Theta}+A_{z 5}^{r}
$$




$$
\begin{array}{r}
A_{z 5}^{\Theta}=\sum_{h 5=1}^{\infty}\left[c 5_{h 5}^{\Theta} \cdot r_{2} \cdot \frac{E_{\phi}\left(\beta 5_{h 5}, r_{3}, r\right)}{E_{\phi}\left(\beta 5_{h 5}, r_{3}, r_{2}\right)}+d 5_{h 5}^{\Theta} \cdot r_{3} \cdot \frac{E_{\phi}\left(\beta 5_{h 5}, r, r_{2}\right)}{E_{\phi}\left(\beta 5_{h 5}, r_{3}, r_{2}\right)}\right] \cdot \sin \left[\beta 5_{h 5} \cdot\left(\Theta-\Theta_{3}\right)\right], \\
A_{z 5}^{r}=\sum_{n 5=1}^{\infty}\left\{e 5_{n 5}^{r} \cdot \frac{\operatorname{sh}\left[\lambda 5_{n 5} \cdot\left(\Theta_{4}-\Theta\right)\right]}{\operatorname{sh}\left(\lambda 5_{n 5} \cdot \tau_{\Theta 5}\right)}+f 5_{n 5}^{r} \cdot \frac{\operatorname{sh}\left[\lambda 5_{n 5} \cdot\left(\Theta-\Theta_{3}\right)\right]}{s h\left(\lambda 5_{n 5} \cdot \tau_{\Theta 5}\right)}\right\} \cdot r_{2} \cdot \sin \left[\lambda 5_{n 5} \cdot \ln \left(\frac{r}{r_{2}}\right)\right],
\end{array}
$$

the $r$-component of $\mathbf{B}_{5}$ by

$$
\begin{gathered}
B_{r 5}=B_{r 5}^{\Theta}+B_{\Theta 5}^{r}, \\
B_{r 5}^{\Theta}=\sum_{h 5=1}^{\infty} \beta 5_{h 5} \cdot\left[c 5_{h 5}^{\Theta} \cdot \frac{r_{2}}{r} \cdot \frac{E_{\phi}\left(\beta 5_{h 5}, r_{3}, r\right)}{E_{\phi}\left(\beta 5_{h 5}, r_{3}, r_{2}\right)}+d 5_{h 5}^{\Theta} \cdot \frac{r_{3}}{r} \cdot \frac{E_{\phi}\left(\beta 5_{h 5}, r, r_{2}\right)}{E_{\phi}\left(\beta 5_{h 5}, r_{3}, r_{2}\right)}\right] \cdot \cos \left[\beta 5_{h 5} \cdot\left(\Theta-\Theta_{3}\right)\right], \\
B_{r 5}^{r}=\sum_{n 5=1}^{\infty} \lambda 5_{n 5} \cdot\left\{-e 5_{n 5}^{r} \cdot \frac{\operatorname{ch}\left[\lambda 5_{n 5} \cdot\left(\Theta_{4}-\Theta\right)\right]}{\operatorname{sh}\left(\lambda 5_{n 5} \cdot \tau_{\Theta 5}\right)}+f 5_{n 5}^{r} \cdot \frac{\operatorname{ch}\left[\lambda 5_{n 5} \cdot\left(\Theta-\Theta_{3}\right)\right]}{\operatorname{sh}\left(\lambda 5_{n 5} \cdot \tau_{\Theta 5}\right)}\right\} \cdot \frac{r_{2}}{r} \cdot \sin \left[\lambda 5_{n 5} \cdot \ln \left(\frac{r}{r_{2}}\right)\right],
\end{gathered}
$$

the $\Theta$-component of $\mathbf{B}_{5}$ by

$$
\begin{gathered}
B_{\Theta 5}=B_{\Theta 5}^{\Theta}+B_{\Theta 5}^{r}, \\
B_{\Theta 5}^{\Theta}=\sum_{h 5=1}^{\infty} \beta 5_{h 5} \cdot\left[c 5_{h 5}^{\Theta} \cdot \frac{r_{2}}{r} \cdot \frac{P_{\phi}\left(\beta 5_{h 5}, r_{3}, r\right)}{E_{\phi}\left(\beta 5_{h 5}, r_{3}, r_{2}\right)}-d 5_{h 5}^{\Theta} \cdot \frac{r_{3}}{r} \cdot \frac{P_{\phi}\left(\beta 5_{h 5}, r, r_{2}\right)}{E_{\phi}\left(\beta 5_{h 5}, r_{3}, r_{2}\right)}\right] \cdot \sin \left[\beta 5_{h 5} \cdot\left(\Theta-\Theta_{3}\right)\right], \\
B_{\Theta 5}^{r}=-\sum_{n 5=1}^{\infty} \lambda 5_{n 5} \cdot\left\{e 5_{n 5}^{r} \cdot \frac{\operatorname{sh}\left[\lambda 5_{n 5} \cdot\left(\Theta_{4}-\Theta\right)\right]}{\operatorname{sh}\left(\lambda 5_{n 5} \cdot \tau_{\Theta 5}\right)}+f 5_{n 5}^{r} \cdot \frac{\operatorname{sh}\left[\lambda 5_{n 5} \cdot\left(\Theta-\Theta_{3}\right)\right]}{s h\left(\lambda 5_{n 5} \cdot \tau_{\Theta 5}\right)}\right\} \cdot \frac{r_{2}}{r} \cdot \cos \left[\lambda 5_{n 5} \cdot \ln \left(\frac{r}{r_{2}}\right)\right],
\end{gathered}
$$

where $h 5$ and $n 5$ are the spatial harmonic orders in Region $5 ; c 5_{h 5}^{\Theta}, d 5_{h 5}^{\Theta}, e 5_{n 5}^{r}$ and $f 5_{n 5}^{r}$ the integration constants; $\beta 5_{h 5}=h 5 \cdot \pi / \tau_{\Theta 5}$ with $\tau_{\Theta 5}=\Theta_{4}-\Theta_{3}$; and $\lambda 5_{n 5}=n 5 \cdot \pi / \tau_{r 5}$ with $\tau_{r 5}=\ln \left(r_{3} / r_{2}\right)$.

Using Fourier series expansion of $\left.A_{z 1}\right|_{\forall \Theta \wedge r=r_{2}}$ and $\left.A_{z 2}\right|_{\forall \Theta \wedge r=r_{3}}$ (see Figure 3e) over the interval $\Theta=\left[\Theta_{3}, \Theta_{4}\right]=\left[\Theta_{3}, \Theta_{3}+\tau_{\Theta 5}\right]$, the integration constants $c 5_{h 5}^{\Theta}$ and $d 5_{h 5}^{\Theta}$ are determined in Appendix $C$ with

$$
\begin{aligned}
& c 5_{h 5}^{\Theta}=\frac{2}{\tau_{\Theta 5}} \cdot \int_{\Theta_{3}}^{\Theta_{3}+\tau_{\Theta 5}} \frac{\left.A_{z 1}\right|_{r=r_{2}}}{r_{2}} \cdot \sin \left[\beta 5_{h 5} \cdot\left(\Theta-\Theta_{3}\right)\right] \cdot d \Theta, \\
& d 5_{h 5}^{\Theta}=\frac{2}{\tau_{\Theta 5}} \cdot \int_{\Theta_{3}}^{\Theta_{3}+\tau_{\Theta 5}} \frac{\left.A_{z 2}\right|_{r=r_{3}}}{r_{3}} \cdot \sin \left[\beta 5_{h 5} \cdot\left(\Theta-\Theta_{3}\right)\right] \cdot d \Theta .
\end{aligned}
$$

With a weighting function $g(r)=r^{-1}$ and using a Fourier series expansion of $\left.A_{z 6}\right|_{\Theta=\Theta_{3} \wedge \forall r}$ and $\left.A_{z 7}\right|_{\Theta=\Theta_{4} \wedge \forall r}$ (see Figure 3e) over the interval $r=\left[r_{2}, r_{3}\right]$, the integration constants $e 5_{n 5}^{r}$ and $f 5_{n 5}^{r}$ are determined in Appendix $C$ with

$$
\begin{aligned}
& e 5_{n 5}^{r}=\frac{2}{\tau_{r 5}} \cdot \int_{r_{2}}^{r_{3}} \frac{1}{r} \cdot \frac{\left.A_{z 6}\right|_{\Theta=\Theta_{3}}}{r_{2}} \cdot \sin \left[\lambda 5_{n 5} \cdot \ln \left(\frac{r}{r_{2}}\right)\right] \cdot d r \\
& f 5_{n 5}^{r}=\frac{2}{\tau_{r 5}} \cdot \int_{r_{2}}^{r_{3}} \frac{1}{r} \cdot \frac{\left.A_{z 7}\right|_{\Theta=\Theta_{4}}}{r_{2}} \cdot \sin \left[\lambda 5_{n 5} \cdot \ln \left(\frac{r}{r_{2}}\right)\right] \cdot d r .
\end{aligned}
$$

\subsubsection{Region 6}

For Region 6, the general solution is given according to the BCs of case-study no 2 (i.e., $B_{r}$ and $A_{z}$ are respectively imposed on $r$ - and $\Theta$-edges of a region) in Appendix $B$. Therefore, $A_{z 6}$ satisfying the BCs of Figure $3 \mathrm{f}$ and solution of $(1 \mathrm{~b})$ is given by

$$
A_{z 6}=A_{z 6}^{\Theta}+A_{z 6}^{r}+A_{z P 6}
$$




$$
\begin{gathered}
A_{z 6}^{\Theta}=\mid \begin{array}{l}
c 6_{0}^{\Theta} \cdot r_{2} \cdot \frac{\ln \left(r_{3} / r\right)}{\ln \left(r_{3} / r_{2}\right)}+d 6_{0}^{\Theta} \cdot r_{3} \cdot \frac{\ln \left(r / r_{2}\right)}{\ln \left(r_{3} / r_{2}\right)} \\
\cdots+\sum_{h 6=1}^{\infty}\left[c 6_{h 6}^{\Theta} \cdot r_{2} \cdot \frac{E_{\phi}\left(\beta 6_{h 6}, r_{3}, r\right)}{E_{\phi}\left(\beta 6_{h 6}, r_{3}, r_{2}\right)}+d 6_{h 6}^{\Theta} \cdot r_{3} \cdot \frac{E_{\phi}\left(\beta 6_{h 6}, r, r_{2}\right)}{E_{\phi}\left(\beta 6_{h 6}, r_{3}, r_{2}\right)}\right] \cdot \cos \left[\beta 6_{h 6} \cdot\left(\Theta-\Theta_{2}\right)\right]
\end{array} \\
A_{z 6}^{r}=\sum_{n 6=1}^{\infty}\left\{e 6_{n 6}^{r} \cdot \frac{\operatorname{ch}\left[\lambda 6_{n 6} \cdot\left(\Theta-\Theta_{2}\right)\right]}{\operatorname{sh}\left(\lambda 6_{n 6} \cdot \tau_{\Theta 6}\right)}-f 6_{n 6}^{r} \cdot \frac{\operatorname{ch}\left[\lambda 6_{n 6} \cdot\left(\Theta_{3}-\Theta\right)\right]}{\operatorname{sh}\left(\lambda 6_{n 6} \cdot \tau_{\Theta 6}\right)}\right\} \cdot \frac{r_{2}}{\lambda 6_{n 6}} \cdot \sin \left[\lambda 6_{n 6} \cdot \ln \left(\frac{r}{r_{2}}\right)\right] .
\end{gathered}
$$

Considering (26b) and (26c) as well as the form of the current density distribution, i.e., (2), a particular solution $A_{z P 6}$ can be found. The following particular solution is proposed

$$
A_{z P 6}=-\frac{1}{4} \cdot r^{2} \cdot \mu_{6} \cdot J_{z 6} .
$$

The $r$-component of $\mathbf{B}_{6}$ is defined by

$$
\begin{gathered}
B_{r 6}=B_{r 6}^{\Theta}+B_{r 6}^{r}+B_{r P 6}, \\
B_{r 6}^{\Theta}=-\sum_{h 6=1}^{\infty} \beta 6_{h 6} \cdot\left[c 6_{h 6}^{\Theta} \cdot \frac{r_{2}}{r} \cdot \frac{E_{\phi}\left(\beta 6_{h 6}, r_{3}, r\right)}{E_{\phi}\left(\beta 6_{h 6}, r_{3}, r_{2}\right)}+d 6_{h 6}^{\Theta} \cdot \frac{r_{3}}{r} \cdot \frac{E_{\phi}\left(\beta 6_{h 6}, r, r_{2}\right)}{E_{\phi}\left(\beta 6_{h 6}, r_{3}, r_{2}\right)}\right] \cdot \sin \left[\beta 6_{h 6} \cdot\left(\Theta-\Theta_{2}\right)\right], \\
B_{r 6}^{r}=\sum_{n 6=1}^{\infty}\left\{e 6_{n 6}^{r} \cdot \frac{\operatorname{sh}\left[\lambda 6_{n 6} \cdot\left(\Theta-\Theta_{2}\right)\right]}{s h\left(\lambda 6_{n 6} \cdot \tau_{\Theta 6}\right)}+f 6_{n 6}^{r} \cdot \frac{s h\left[\lambda 6_{n 6} \cdot\left(\Theta_{3}-\Theta\right)\right]}{s h\left(\lambda 6_{n 6} \cdot \tau_{\Theta 6}\right)}\right\} \cdot \frac{r_{2}}{r} \cdot \sin \left[\lambda 6_{n 6} \cdot \ln \left(\frac{r}{r_{2}}\right)\right], \\
B_{r P 6}=\frac{1}{r} \cdot \frac{\partial A_{z P 6}}{\partial \Theta}=0,
\end{gathered}
$$

and the $\Theta$-component of $\mathbf{B}_{6}$ by

$$
B_{\Theta 6}=B_{\Theta 6}^{\Theta}+B_{\Theta 6}^{r}+B_{\Theta P 6}
$$

$$
\begin{gathered}
B_{\Theta 6}^{\Theta}=\mid \begin{array}{c}
c 6_{0}^{\Theta} \cdot \frac{r_{2}}{r} \cdot \frac{1}{\ln \left(r_{3} / r_{2}\right)}-d 6_{0}^{\Theta} \cdot \frac{r_{3}}{r} \cdot \frac{1}{\ln \left(r_{3} / r_{2}\right)} \\
\cdots+\sum_{h 6=1}^{\infty} \beta 6_{h 6} \cdot\left[c 6_{h 6}^{\Theta} \cdot \frac{r_{2}}{r} \cdot \frac{P_{\phi}\left(\beta 6_{h 6}, r_{3}, r\right)}{E_{\phi}\left(\beta 6_{h 6}, r_{3}, r_{2}\right)}-d 6_{h 6}^{\Theta} \cdot \frac{r_{3}}{r} \cdot \frac{P_{q}\left(\beta 6_{h 6}, r, r_{2}\right)}{E_{q}\left(\beta 6_{h 6}, r_{3}, r_{2}\right)}\right] \cdot \cos \left[\beta 6_{h 6} \cdot\left(\Theta-\Theta_{2}\right)\right]
\end{array}, \\
B_{\Theta 6}^{r}=-\sum_{n 6=1}^{\infty}\left\{e 6_{n 6}^{r} \cdot \frac{c h\left[\lambda 6_{n 6} \cdot\left(\Theta-\Theta_{2}\right)\right]}{\operatorname{sh}\left(\lambda 6_{n 6} \cdot \tau_{\Theta 6}\right)}-f 6_{n 6}^{r} \cdot \frac{\operatorname{ch}\left[\lambda 6_{n 6} \cdot\left(\Theta_{3}-\Theta\right)\right]}{\operatorname{sh}\left(\lambda 6_{n 6} \cdot \tau_{\Theta 6}\right)}\right\} \cdot \frac{r_{2}}{r} \cdot \cos \left[\lambda 6_{n 6} \cdot \ln \left(\frac{r}{r_{2}}\right)\right], \\
B_{\Theta P 6}=-\frac{\partial A_{z P 6}}{\partial r}=\frac{1}{2} \cdot r \cdot \mu_{6} \cdot J_{z 6},
\end{gathered}
$$

where $h 6$ and $n 6$ are the spatial harmonic orders in Region $6 ; c 6_{0}^{\Theta}, d 6_{0}^{\Theta}, c 6_{h 6^{\prime}}^{\Theta} d 6_{h 6^{\prime}}^{\Theta} e 6_{n 6}^{r}$ and $f 6_{n 6}^{r}$ the integration constants; $\beta 6_{h 6}=h 6 \cdot \pi / \tau_{\Theta 6}$ with $\tau_{\Theta 6}=\Theta_{3}-\Theta_{2}$; and $\lambda 6_{n 6}=n 6 \cdot \pi / \tau_{r 6}$ with $\tau_{r 6}=\ln \left(r_{3} / r_{2}\right)$.

Using Fourier series expansion of $\left.A_{z 1}\right|_{\forall \Theta \wedge r=r_{2}}$ and $\left.A_{z 2}\right|_{\forall \Theta \wedge r=r_{3}}$ (see Figure 3f) over the interval $\Theta=\left[\Theta_{2}, \Theta_{3}\right]=\left[\Theta_{2}, \Theta_{2}+\tau_{\Theta 6}\right]$, the integration constants $c 6_{0}^{\Theta} \& c 6_{h 6}^{\Theta}$ and $d 6_{0}^{\Theta} \& d 6_{h 6}^{\Theta}$ are determined in Appendix $\mathrm{C}$ with

$$
\begin{gathered}
c 6_{0}^{\Theta}=\frac{1}{\tau_{\Theta 6}} \cdot \int_{\Theta_{2}}^{\Theta_{2}+\tau_{\Theta 6}} \frac{1}{r_{2}} \cdot\left[\left.A_{z 1}\right|_{r=r_{2}}-\left.A_{z P 6}\right|_{r=r_{2}}\right] \cdot d \Theta, \\
c 6_{h 6}^{x}=\frac{2}{\tau_{\Theta 6}} \cdot \int_{\Theta_{2}}^{\Theta_{2}+\tau_{\Theta 6}} \frac{1}{r_{2}} \cdot\left[\left.A_{z 1}\right|_{r=r_{2}}-\left.A_{z P 6}\right|_{r=r_{2}}\right] \cdot \cos \left[\beta 6_{h 6} \cdot\left(\Theta-\Theta_{2}\right)\right] \cdot d \Theta, \\
d 6_{0}^{\Theta}=\frac{1}{\tau_{\Theta 6}} \cdot \int_{\Theta_{2}}^{\Theta_{2}+\tau_{\Theta 6}} \frac{1}{r_{3}} \cdot\left[\left.A_{z 2}\right|_{r=r_{3}}-\left.A_{z P 6}\right|_{r=r_{3}}\right] \cdot d \Theta,
\end{gathered}
$$




$$
d 6_{h 6}^{x}=\frac{2}{\tau_{\Theta 6}} \cdot \int_{\Theta_{2}}^{\Theta_{2}+\tau_{\Theta 6}} \frac{1}{r_{3}} \cdot\left[\left.A_{z 2}\right|_{r=r_{3}}-\left.A_{z P 6}\right|_{r=r_{3}}\right] \cdot \cos \left[\beta 6_{h 6} \cdot\left(\Theta-\Theta_{2}\right)\right] \cdot d \Theta
$$

Using a Fourier series expansion of $\mu_{6} /\left.\mu_{5} \cdot B_{r 5}\right|_{\Theta=\Theta_{3} \wedge \forall r}$ and $\mu_{6} /\left.\mu_{3} \cdot B_{r 3}\right|_{\Theta=\Theta_{2} \wedge \forall r}$ (see Figure 3f) over the interval $r=\left[r_{2}, r_{3}\right]$, the integration constants $e 6_{n 6}^{r}$ and $f 6_{n 6}^{r}$ are determined in Appendix $C$ with

$$
\begin{aligned}
& e 6_{n 6}^{r}=\frac{2}{\tau_{r 6}} \cdot \int_{r_{2}}^{r_{3}} \frac{1}{r_{2}} \cdot\left[\left.\frac{\mu_{6}}{\mu_{5}} \cdot B_{r 5}\right|_{\Theta=\Theta_{3}}-\left.B_{r P 6}\right|_{\Theta=\Theta_{3}}\right] \cdot \sin \left[\lambda 6_{n 6} \cdot \ln \left(\frac{r}{r_{2}}\right)\right] \cdot d r, \\
& f 6_{n 6}^{r}=\frac{2}{\tau_{r 6}} \cdot \int_{r_{2}}^{r_{3}} \frac{1}{r_{2}} \cdot\left[\left.\frac{\mu_{6}}{\mu_{3}} \cdot B_{r 3}\right|_{\Theta=\Theta_{2}}-\left.B_{r P 6}\right|_{\Theta=\Theta_{2}}\right] \cdot \sin \left[\lambda 6_{n 6} \cdot \ln \left(\frac{r}{r_{2}}\right)\right] \cdot d r .
\end{aligned}
$$

\subsubsection{Region 7}

The solution in Region 7 is using the same development than Region 6. Thus, $A_{z 7}$ satisfying the BCs of Figure $3 g$ and solution of (2) is defined by

$$
\begin{gathered}
A_{z 7}=A_{z 7}^{\Theta}+A_{z 7}^{r}+A_{z P 7}, \\
A_{z 7}^{\Theta}=\mid \begin{array}{c}
c 7_{0}^{\Theta} \cdot r_{2} \cdot \frac{\ln \left(r_{3} / r\right)}{\ln \left(r_{3} / r_{2}\right)}+d 7_{0}^{\Theta} \cdot r_{3} \cdot \frac{\ln \left(r / r_{2}\right)}{\ln \left(r_{3} / r_{2}\right)} \\
\cdots+\sum_{h 7=1}^{\infty}\left[c 7_{h 7}^{\Theta} \cdot r_{2} \cdot \frac{E_{\phi}\left(\beta 7_{h 7}, r_{3}, r\right)}{E_{\phi}\left(\beta 7_{h 7}, r_{3}, r_{2}\right)}+d 7_{h 7}^{\Theta} \cdot r_{3} \cdot \frac{E_{\phi}\left(\beta 7_{h 7}, r, r_{2}\right)}{E_{\phi}\left(\beta 7_{h 7}, r_{3}, r_{2}\right)}\right] \cdot \cos \left[\beta 7_{h 7} \cdot\left(\Theta-\Theta_{4}\right)\right]
\end{array} \\
A_{z 7}^{r}=\sum_{n 7=1}^{\infty}\left\{e 7_{n 7}^{r} \cdot \frac{c h\left[\lambda 7_{n 7} \cdot\left(\Theta-\Theta_{4}\right)\right]}{s h\left(\lambda 7_{n 7} \cdot \tau_{\Theta 7}\right)}-f 7_{n 7}^{r} \cdot \frac{\operatorname{ch}\left[\lambda 7_{n 7} \cdot\left(\Theta_{5}-\Theta\right)\right]}{s h\left(\lambda 7_{n 7} \cdot \tau_{\Theta 7}\right)}\right\} \cdot \frac{r_{2}}{\lambda 7_{n 7}} \cdot \sin \left[\lambda 7_{n 7} \cdot \ln \left(\frac{r}{r_{2}}\right)\right], \\
A_{z P 7}=-\frac{1}{4} \cdot r^{2} \cdot \mu_{7} \cdot J_{z 7} .
\end{gathered}
$$

The $r$-component of $\mathbf{B}_{7}$ is defined by

$$
\begin{gathered}
B_{r 7}=B_{r 7}^{\Theta}+B_{r 7}^{r}+B_{r P 7}, \\
B_{r 7}^{\Theta}=-\sum_{h 7=1}^{\infty} \beta 7_{h 7} \cdot\left[c 7_{h 7}^{\Theta} \cdot \frac{r_{2}}{r} \cdot \frac{E_{\phi}\left(\beta 7_{h 7,}, r_{3}, r\right)}{E_{\phi}\left(\beta 7_{h 7}, r_{3}, r_{2}\right)}+d 7_{h 7}^{\Theta} \cdot \frac{r_{3}}{r} \cdot \frac{E_{\phi}\left(\beta 7_{h 7}, r, r_{2}\right)}{E_{\phi}\left(\beta 7_{h 7}, r_{3}, r_{2}\right)}\right] \cdot \sin \left[\beta 7_{h 7} \cdot\left(\Theta-\Theta_{4}\right)\right], \\
B_{r 7}^{r}=\sum_{n 7=1}^{\infty}\left\{e 7_{n 7}^{r} \cdot \frac{s h\left[\lambda 7_{n 7} \cdot\left(\Theta-\Theta_{4}\right)\right]}{s h\left(\lambda 7_{n 7} \cdot \tau_{\Theta 7}\right)}+f 7_{n 7}^{r} \cdot \frac{s h\left[\lambda 7_{n 7} \cdot\left(\Theta_{5}-\Theta\right)\right]}{s h\left(\lambda 7_{n 7} \cdot \tau_{\Theta 7}\right)}\right\} \cdot \frac{r_{2}}{r} \cdot \sin \left[\lambda 7_{n 7} \cdot \ln \left(\frac{r}{r_{2}}\right)\right], \\
B_{r P 7}=\frac{1}{r} \cdot \frac{\partial A_{z P 7}}{\partial \Theta}=0,
\end{gathered}
$$

and the $\Theta$-component of $\mathbf{B}_{7}$ by

$$
\begin{gathered}
B_{\Theta 7}=B_{\Theta 7}^{\Theta}+B_{\Theta 7}^{r}+B_{\Theta P 7}, \\
B_{\Theta 7}^{\Theta}=\mid \begin{array}{c}
c 7_{0}^{\Theta} \cdot \frac{r_{2}}{r} \cdot \frac{1}{\ln \left(r_{3} / r_{2}\right)}-d 7_{0}^{\Theta} \cdot \frac{r_{3}}{r} \cdot \frac{1}{\ln \left(r_{3} / r_{2}\right)} \\
\cdots+\sum_{h 7=1}^{\infty} \beta 7_{h 7} \cdot\left[c 7_{h 7}^{\Theta} \cdot \frac{r_{2}}{r} \cdot \frac{P_{\phi}\left(\beta 7_{h 7}, r_{3}, r\right)}{E_{\phi}\left(\beta 7_{h 7}, r_{3}, r_{2}\right)}-d 7_{h 7}^{\Theta} \cdot \frac{r_{3}}{r} \cdot \frac{P_{\phi}\left(\beta 7_{h 7}, r, r_{2}\right)}{E_{\phi}\left(\beta 7_{h 7}, r_{3}, r_{2}\right)}\right] \cdot \cos \left[\beta 7_{h 7} \cdot\left(\Theta-\Theta_{4}\right)\right]
\end{array}, \\
B_{\Theta 7}^{r}=-\sum_{n 7=1}^{\infty}\left\{e 7_{n 7}^{r} \cdot \frac{c h\left[\lambda 7_{n 7} \cdot\left(\Theta-\Theta_{4}\right)\right]}{s h\left(\lambda 7_{n 7} \cdot \tau_{\Theta 7}\right)}-f 7_{n 7}^{r} \cdot \frac{c h\left[\lambda 7_{n 7} \cdot\left(\Theta_{5}-\Theta\right)\right]}{s h\left(\lambda 7_{n 7} \cdot \tau_{\Theta 7}\right)}\right\} \cdot \frac{r_{2}}{r} \cdot \cos \left[\lambda 7_{n 7} \cdot \ln \left(\frac{r}{r_{2}}\right)\right], \\
B_{\Theta P 7}=-\frac{\partial A_{z P 7}}{\partial r}=\frac{1}{2} \cdot r \cdot \mu_{7} \cdot J_{z 7},
\end{gathered}
$$

where $h 7$ and $n 7$ are the spatial harmonic orders in Region $7 ; c 7_{0}^{\Theta}, d 7_{0}^{\Theta}, c 7_{h 7^{\Theta}}^{\Theta} d 7_{h 7^{\Theta}}^{\Theta} e 7_{n 7}^{r}$ and $f 7_{n 7}^{r}$ the integration constants; $\beta 7_{h 7}=h 7 \cdot \pi / \tau_{\Theta 7}$ with $\tau_{\Theta 7}=\Theta_{5}-\Theta_{4}$; and $\lambda 7_{n 7}=n 7 \cdot \pi / \tau_{r 7}$ with $\tau_{r 7}=\ln \left(r_{3} / r_{2}\right)$. 
Using Fourier series expansion of $\left.A_{z 1}\right|_{\forall \Theta \wedge r=r_{2}}$ and $\left.A_{z 2}\right|_{\forall \Theta \wedge r=r_{3}}$ (see Figure $3 g$ ) over the interval $\Theta=\left[\Theta_{4}, \Theta_{5}\right]=\left[\Theta_{4}, \Theta_{4}+\tau_{\Theta 7}\right]$, the integration constants $c 7_{0}^{\Theta} \& c 7_{h 7}^{\Theta}$ and $d 7_{0}^{\Theta} \& d 7_{h 7}^{\Theta}$ are determined in Appendix $\mathrm{C}$ with

$$
\begin{gathered}
c 7_{0}^{\Theta}=\frac{1}{\tau_{\Theta 7}} \cdot \int_{\Theta_{4}}^{\Theta_{4}+\tau_{\Theta 7}} \frac{1}{r_{2}} \cdot\left[\left.A_{z 1}\right|_{r=r_{2}}-\left.A_{z P 7}\right|_{r=r_{2}}\right] \cdot d \Theta, \\
c 7_{h 7}^{x}=\frac{2}{\tau_{\Theta 7}} \cdot \int_{\Theta_{4}}^{\Theta_{4}+\tau_{\Theta 7}} \frac{1}{r_{2}} \cdot\left[\left.A_{z 1}\right|_{r=r_{2}}-\left.A_{z P 7}\right|_{r=r_{2}}\right] \cdot \cos \left[\beta 7_{h 7} \cdot\left(\Theta-\Theta_{4}\right)\right] \cdot d \Theta, \\
d 7_{0}^{\Theta}=\frac{1}{\tau_{\Theta 7}} \cdot \int_{\Theta_{4}}^{\Theta_{4}+\tau_{\Theta 7}} \frac{1}{r_{3}} \cdot\left[\left.A_{z 2}\right|_{r=r_{3}}-\left.A_{z P 7}\right|_{r=r_{3}}\right] \cdot d \Theta, \\
d 7_{h 7}^{x}=\frac{2}{\tau_{\Theta 7}} \cdot \int_{\Theta_{4}}^{\Theta_{4}+\tau_{\Theta 7}} \frac{1}{r_{3}} \cdot\left[\left.A_{z 2}\right|_{r=r_{3}}-\left.A_{z P 7}\right|_{r=r_{3}}\right] \cdot \cos \left[\beta 7_{h 7} \cdot\left(\Theta-\Theta_{4}\right)\right] \cdot d \Theta .
\end{gathered}
$$

Using a Fourier series expansion of $\mu_{7} /\left.\mu_{4} \cdot B_{r 4}\right|_{\Theta=\Theta_{5} \wedge \forall r}$ and $\mu_{7} /\left.\mu_{5} \cdot B_{r 5}\right|_{\Theta=\Theta_{4} \wedge \forall r}$ (see Figure 3g) over the interval $r=\left[r_{2}, r_{3}\right]$, the integration constants $e 7_{n 7}^{r}$ and $f 7_{n 7}^{r}$ are determined in Appendix $C$ with

$$
\begin{aligned}
& e 7_{n 7}^{r}=\frac{2}{\tau_{r 7}} \cdot \int_{r_{2}}^{r_{3}} \frac{1}{r_{2}} \cdot\left[\left.\frac{\mu_{7}}{\mu_{4}} \cdot B_{r 4}\right|_{\Theta=\Theta_{5}}-\left.B_{r P 7}\right|_{\Theta=\Theta_{5}}\right] \cdot \sin \left[\lambda 7_{n 7} \cdot \ln \left(\frac{r}{r_{2}}\right)\right] \cdot d r, \\
& f 7_{n 7}^{r}=\frac{2}{\tau_{r 7}} \cdot \int_{r_{2}}^{r_{3}} \frac{1}{r_{2}} \cdot\left[\left.\frac{\mu_{7}}{\mu_{5}} \cdot B_{r 5}\right|_{\Theta=\Theta_{4}}-\left.B_{r P 7}\right|_{\Theta=\Theta_{4}}\right] \cdot \sin \left[\lambda 7_{n 7} \cdot \ln \left(\frac{r}{r_{2}}\right)\right] \cdot d r .
\end{aligned}
$$

\section{Validation of the Semi-Analytic Method with FEA}

\subsection{Introduction}

The objective of this section is to validate the proposed 2-D subdomain method in polar coordinates $(r, \Theta)$ on the magnetic field distribution in relation to the numerical method. The physical and geometrical parameters of studied electromagnetic device are given in Table 1.

For this validation, the air- or iron-cored coil has been modeled using Cedrat's Flux2D (Version 10.2.1., Altair Engineering, Meylan Cedex, France) software package (i.e., an advanced finite-element method based numeric field analysis program) [40]. The finite-element model is done with the same assumptions as in the semi-analytical model (see $\$ 2.1$. Model Description and Assumptions). The linear system (i.e., Cramer's system), given in Appendix C, has been implemented in Matlab® (R2015a, Mathworks, Natick, MA, USA) by using the sparse matrix/vectors. A discussion on the numerical problems (viz., harmonics and ill-conditioned systems) of such semi-analytical models has been clarified in [1]. The Maxwell-Fourier methods exhibit a similar problem to the numerical methods due to the periodicity of Fourier series, and consequently to the finite number of harmonics. Hence, $A_{z}$ and $\mathbf{B}=\left\{B_{r} ; B_{\Theta} ; 0\right\}$ in the various regions (see $\S 2.5$. General Solutions of Various Regions) have been computed with a finite number of spatial harmonics terms $H 1_{\max }-H 7_{\max }$ (for the $\Theta$-edges) and $N 3_{\max }-N 7_{\max }$ (for the $r$-edges). As indicated in [41,42], these spatial harmonics terms, given in Table 1, have been imposed according to an optinal ratio, i.e., for $H 1_{\text {max }}$ given,

$$
H \bullet_{\max }=H 1_{\max } \cdot \frac{\tau_{\Theta \bullet}}{\tau_{\Theta 1}} \text { and } N \bullet_{\max }=H \bullet_{\text {max }} \cdot \frac{\tau_{\Theta \bullet}}{\tau_{r \bullet}} .
$$


Table 1. Physical and Geometrical Parameters of the Air- or Iron-Cored Coil.

\begin{tabular}{cc}
\hline Parameters, Symbols [Units] & Values \\
\hline Number of turns of the coil, $N_{t}[-]$ & 60 \\
Supply current, $I[\mathrm{~A}]$ & 20 \\
Conductor Surface, $S_{c}\left[\mathrm{~mm}^{2}\right]$ & 120 \\
Current density of the coil, $J_{z k}\left[\mathrm{~A} / \mathrm{mm}^{2}\right]$ & \pm 10 \\
Effective axial length, $L_{z}[\mathrm{~mm}]$ & 60 \\
Geometrical parameters in the $\Theta$-axis, $\left\{\Theta_{1} ; \Theta_{2} ; \Theta_{3} ; \Theta_{4} ; \Theta_{5} ; \Theta_{6}\right\}[$ deg.] & $\{0 ; 17 ; 21 ; 29 ; 33 ; 50\}$ \\
Geometrical parameters in the $r$-axis, $\left\{r_{1} ; r_{2} ; r_{3} ; r_{4}\right\}[\mathrm{mm}]$ & $\{21 ; 81 ; 100 ; 160\}$ \\
Relative magnetic permeability of the iron, $\mu_{\text {iron }}[-]$ & 1,500 \\
Number of harmonics for Region $1, H 1_{\max }[-]$ & 260 \\
Number of harmonics for Region $2, H 2_{\max }[-]$ & 260 \\
Number of harmonics for Region $3,\left\{H 3_{\max } ; N 3_{\max }\right\}[-]$ & $\{88 ; 124\}$ \\
Number of harmonics for Region $4,\left\{H 4_{\max } ; N 4_{\max }\right\}[-]$ & $\{88 ; 124\}$ \\
Number of harmonics for Region $5,\left\{H 5_{\max } ; N 5_{\max }\right\}[-]$ & $\{42 ; 124\}$ \\
Number of harmonics for Region $6,\left\{H 6_{\max } ; N 6_{\max }\right\}[-]$ & $\{21 ; 124\}$ \\
Number of harmonics for Region $7,\left\{H 7_{\max } ; N 7_{\max }\right\}[-]$ & $\{21 ; 124\}$ \\
\hline
\end{tabular}

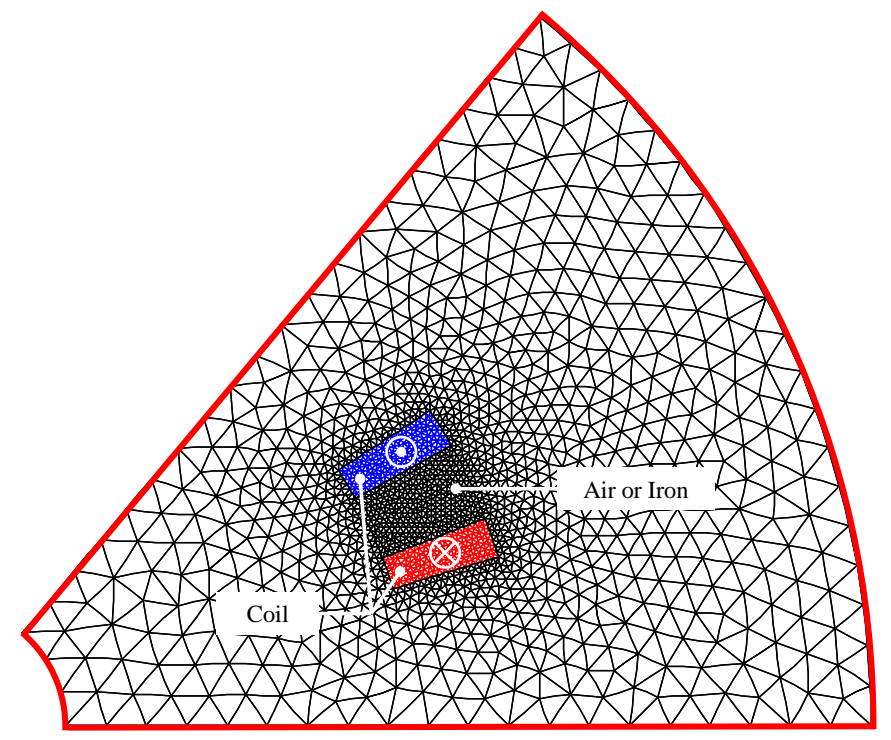

Figure 4. 2-D finite-element analysis (FEA) mesh for the air- or iron-cored coil.

The linear system size depends on the number of: (i) regions; (ii) BCs; and (iii) harmonics of each subdomain. In our study, the linear system (C.3) consists of 2,036 elements which is much smaller than the 2-D FEA mesh having 3,081 surfaces elements of second order (viz., the triangles number of system). For information, the 2-D FEA mesh for an air- or iron-cored coil is illustrated in Figure 4 . The personal computer used for this comparison has the following characteristics: HP Z800 Intel(R) Xeon(R) CPU@2.4 GHz (with 2 processors) RAM 16 Go 64 bits. The computation time of 2-D subdomain model is divided by 2 (viz., $0.5 \mathrm{sec}$ for 2-D subdomain model and $1 \mathrm{sec}$ for the 2-D FEA).

\subsection{Results Discussion}

The validation paths of $A_{z}$ and $\mathbf{B}=\left\{B_{r} ; B_{\Theta} ; 0\right\}$ for the semi-analytic and numeric comparison are given in Figure 5.

The waveforms of global quantities are shown on different paths in Figure 6 for $A_{z}$ and in Figure 7-11 for the components of $\mathbf{B}$. The solid lines represent the global quantities computed by the 2-D FEA and the circles correspond to 2-D subdomain model. Comparing those results with 2-D FEA, 


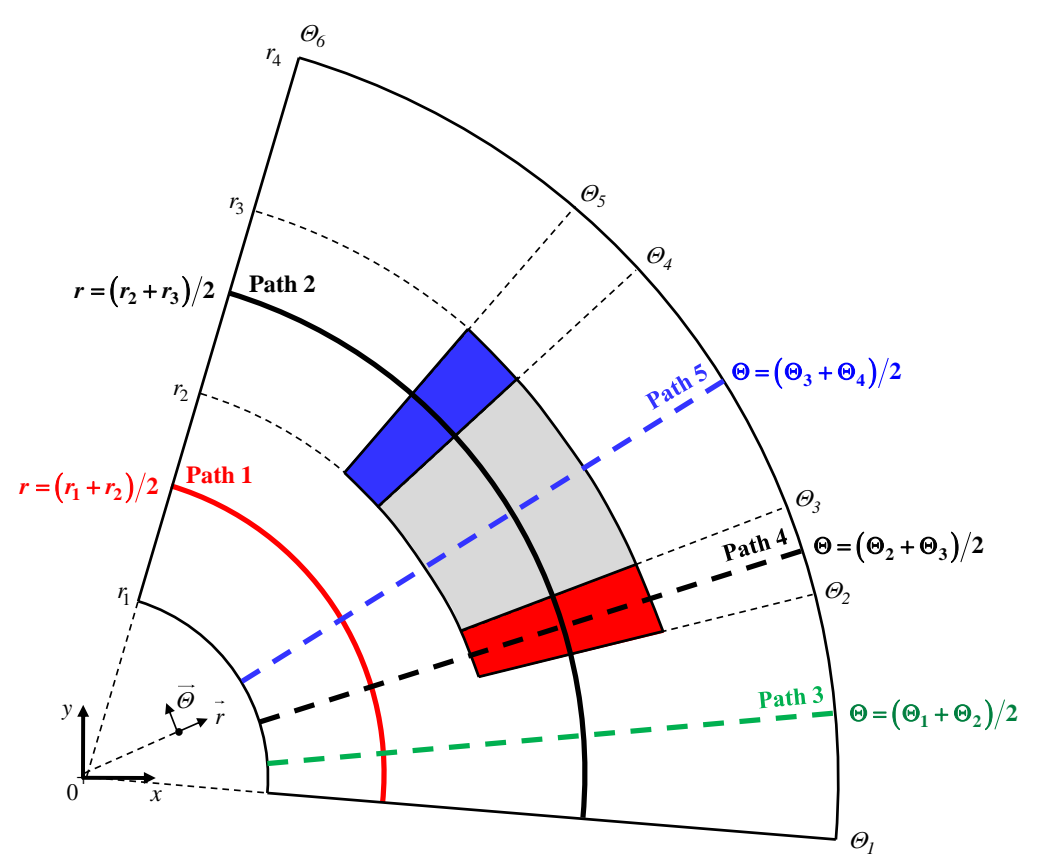

Figure 5. Validation paths for the semi-analytic and numeric comparison.

it can be shown that a very good evaluation is obtained for $A_{z}$ and for the components of $\mathbf{B}$, whatever the paths, for both air- and iron-core. This confirms that the effect of global saturation can be taken into account accurately. It is interesting to note that numerical peaks appear in the FEA results (see Figure 6e, Figure 7, Figure $8 \mathrm{~b}$ and Figure $11 \mathrm{~b}$ ) which are mainly due to the mesh. The relative error is less than $1.5 \%$ for the various global quantities (see Figure $6 a$ and $6 c$ for the maximum error).

\section{Conclusion}

It has been demonstrated that there exists no exact semi-analytical model based on the subdomain technique in polar coordinates taking into account of iron parts with(out) the nonlinear $B(H)$ curve. An improved 2-D subdomain method in polar coordinates $(r, \Theta)$ to study the magnetic field distribution in the iron parts with a finite relative permeability have been presented in this paper. Nevertheless, the research work is an extension of [1] in polar coordinates $(r, \Theta)$.

The proposed new subdomain model is applied to an air- or iron-cored coil supplied by a constant current. The magnetic field solutions in the subdomains and interfaces conditions between regions are carried out in the two directions (i.e., $r$ - and $\Theta$-axis). The iron relative permeability used in this model is constant and corresponds to the linear part of the nonlinear $B(H)$ curve. However, the whole $B(H)$ curve of the magnetic material can be applied with an iterative algorithm as in $[29,30,34]$. The proposed subdomain method in polar coordinates $(r, \Theta)$ takes less computing time than the FEA (approximately 2 fold versus to FEA). It is very suitable for design and optimisation of the electromechanical systems in general and electrical machines in particuler. The semi-analytical results have been validated with FEA and good agreement has been obtained in both amplitudes and waveforms.

The major scientific contributionis the 2-D semi-analytical analysis of the rotating electrical machines (e.g., radial-flux machines, etc.) with(out) magnets supplied by a direct or alternate current (with any waveforms). 


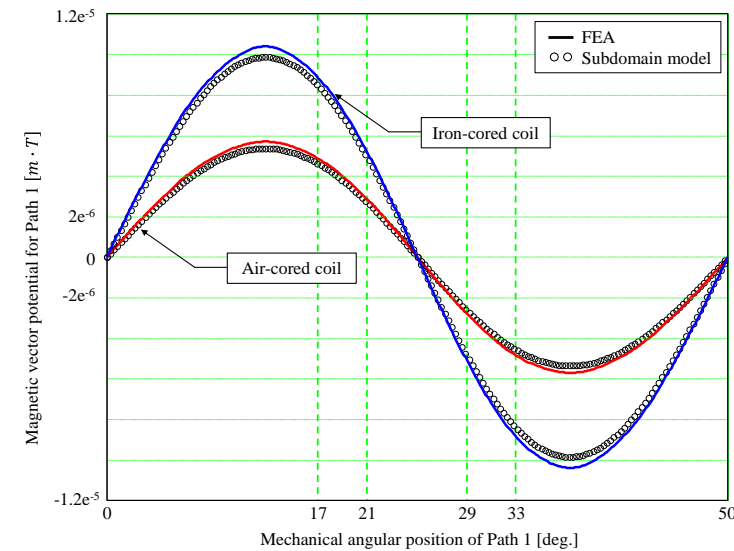

(a)

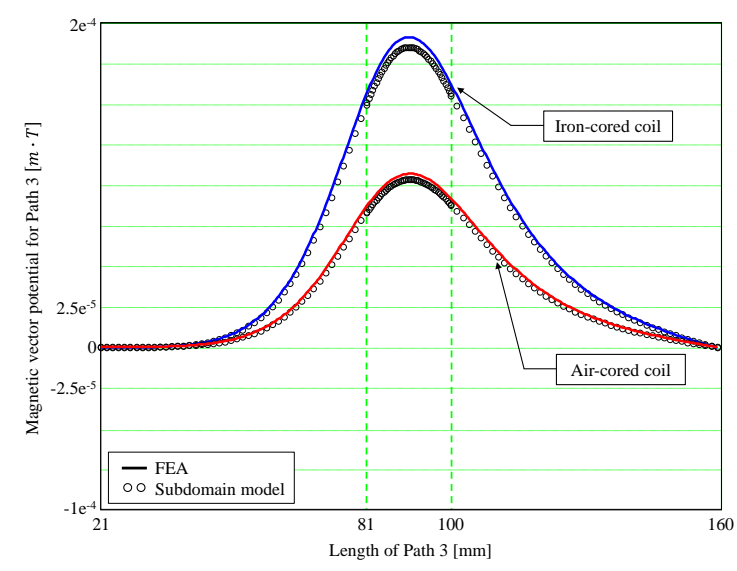

(c)

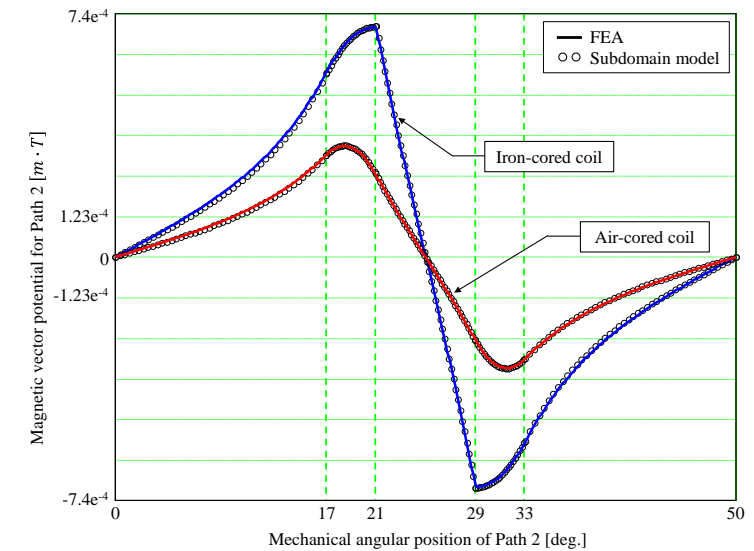

(b)

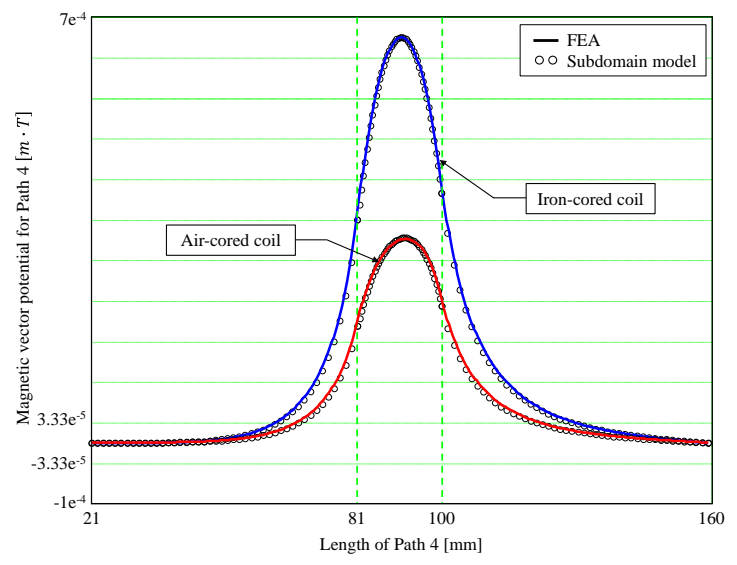

(d)

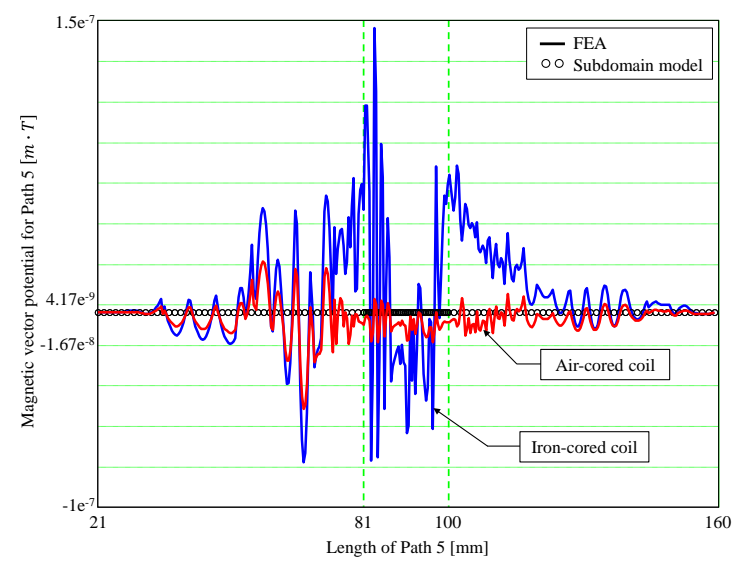

(e)

Figure 6. Waveform of $A_{z}$ for: (a) Path 1, (b) Path 2, (c) Path 3, (d) Path 4, and (e) Path 5. 


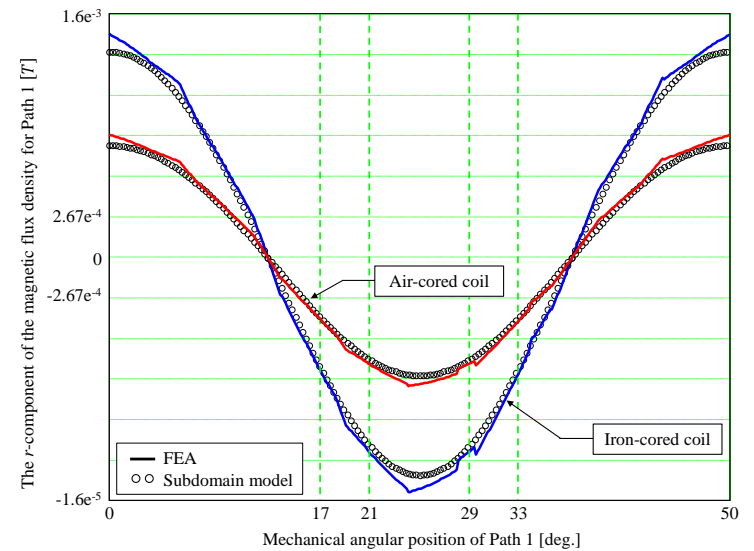

(a)

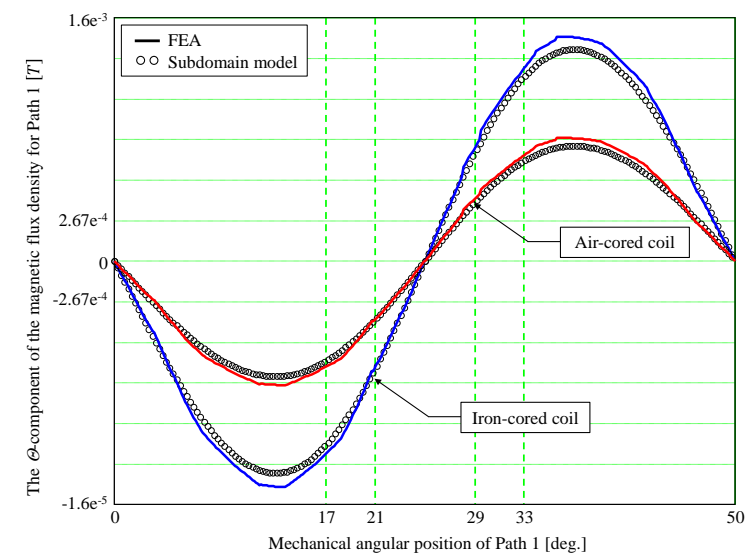

(b)

Figure 7. Waveform of B for Path 1: (a) $r$ - and (b) $\Theta$-component.

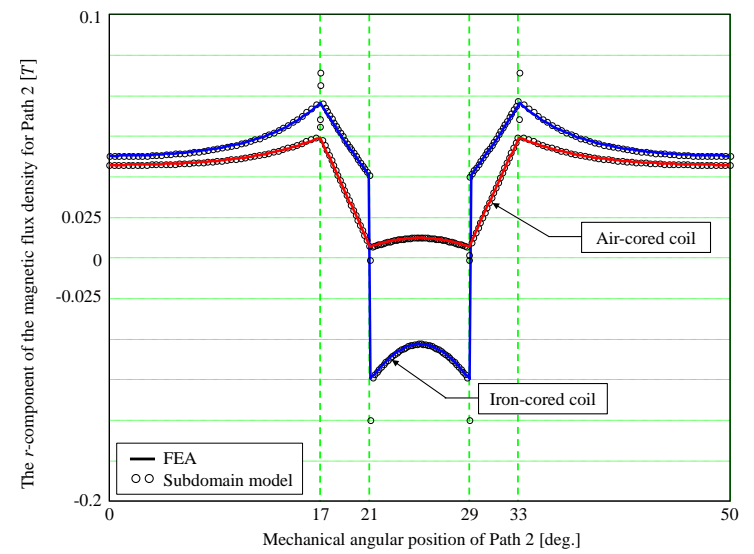

(a)

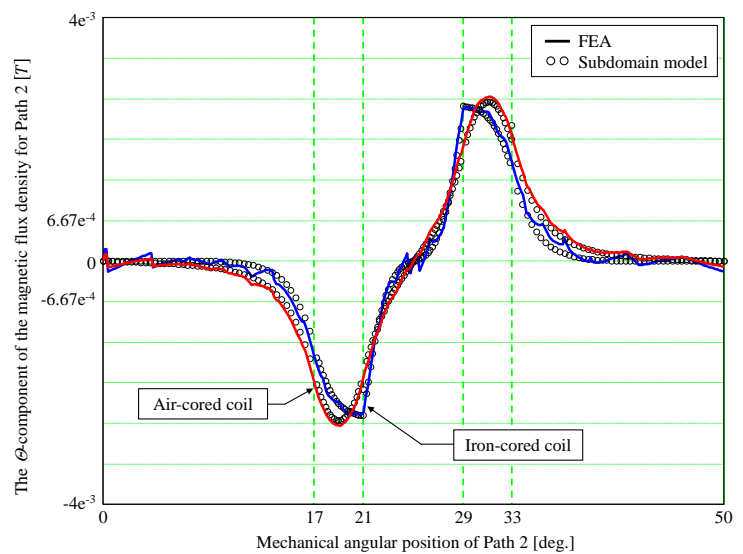

(b)

Figure 8. Waveform of $\mathbf{B}$ for Path 2: (a) $r$ - and (b) $\Theta$-component.

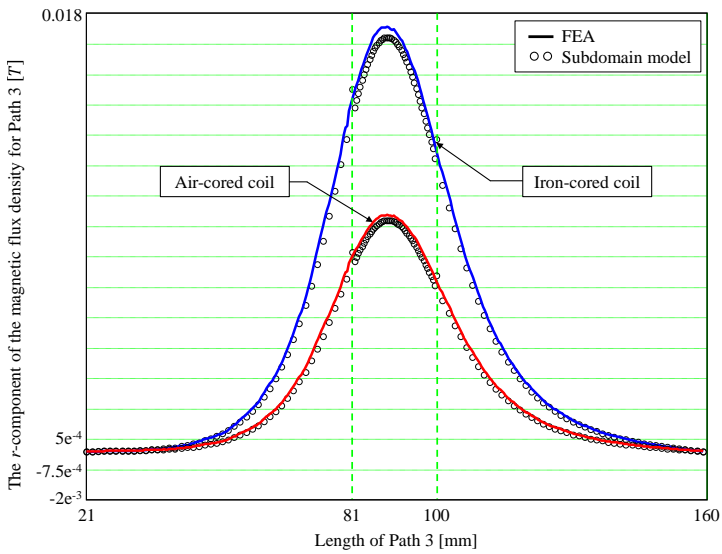

(a)

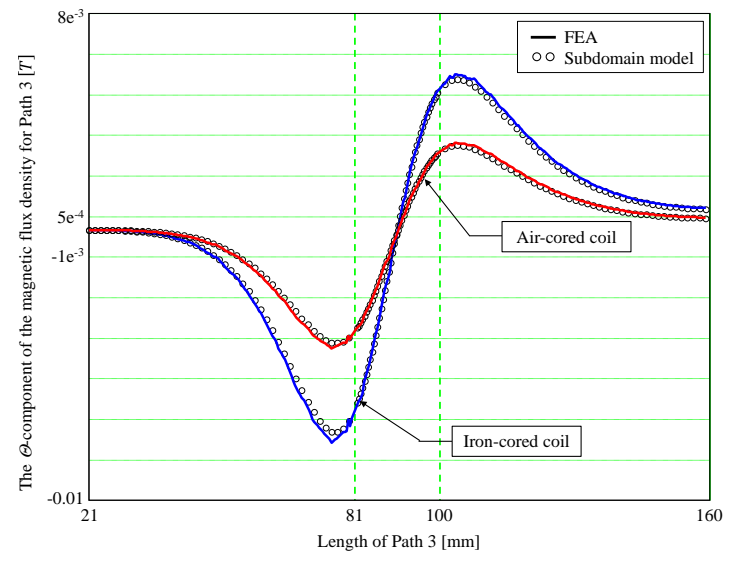

(b)

Figure 9. Waveform of $\mathbf{B}$ for Path 3: (a) $r$ - and (b) $\Theta$-component. 


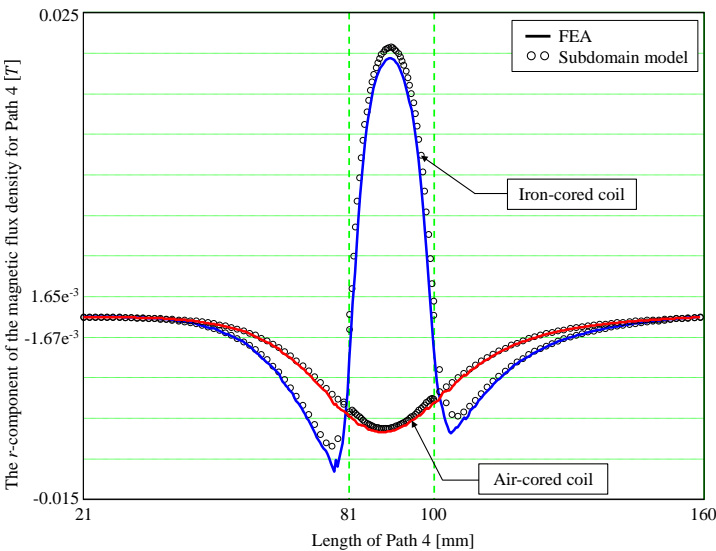

(a)

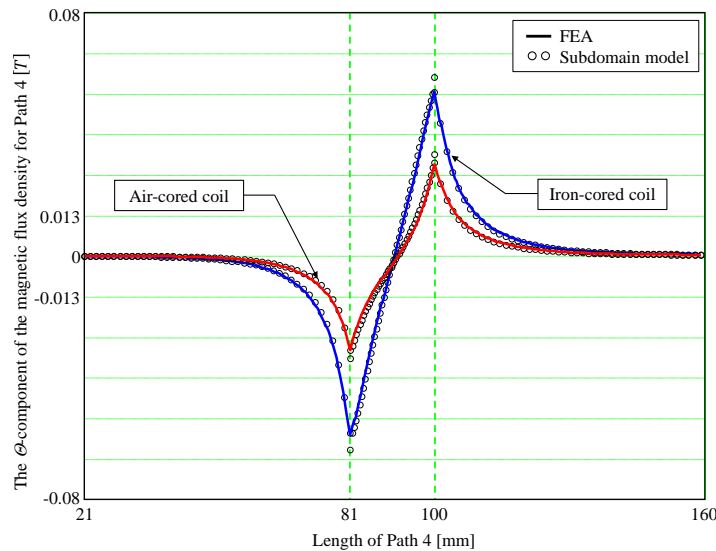

(b)

Figure 10. Waveform of $\mathbf{B}$ for Path 4: (a) $r$ - and (b) $\Theta$-component.

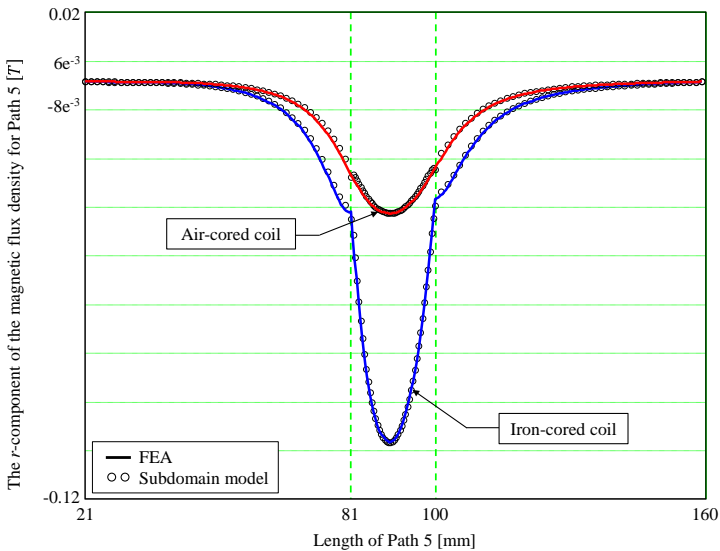

(a)

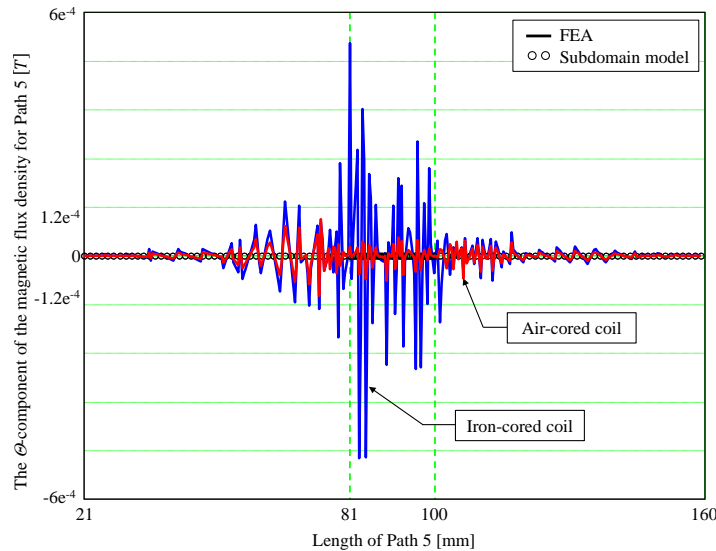

(b)

Figure 11. Waveform of $\mathbf{B}$ for Path 5: (a) $r$ - and (b) $\Theta$-component.

Author Contributions: The work presented here was carried out in cooperation among all authors, which have written the paper and have gave advice for the manuscripts.

Conflicts of Interest: The authors declare no conflict of interest.

\section{Appendix A The 2-D General Solution of EDPs (i.e., Laplace's and Poisson's equations) in Polar Coordinates}

Using the magnetostatic Maxwell's equations (viz., the Maxwell-Ampere law, the Maxwell-Thomson law, and the magnetic material equation) [1], the general EDPs in terms of magnetic vector potential $\mathbf{A}=\left\{0 ; 0 ; A_{z}\right\}$ with $\mu=C^{s t}$ can be expressed in polar coordinates $(r, \Theta)$ by

$$
\begin{gathered}
\Delta A_{z}=\frac{\partial^{2} A_{z}}{\partial r^{2}}+\frac{1}{r} \cdot \frac{\partial A_{z}}{\partial r}+\frac{1}{r^{2}} \cdot \frac{\partial^{2} A_{z}}{\partial \Theta^{2}}=E S, \\
E S=-\left[\mu \cdot J_{z}+\frac{\mu_{0}}{r} \cdot\left(M_{\Theta}+r \cdot \frac{\partial M_{\Theta}}{\partial r}-\frac{\partial M_{r}}{\partial \Theta}\right)\right] .
\end{gathered}
$$

where $\mathbf{J}=\left\{0 ; 0 ; J_{z}\right\}$ is the current density (due to supply currents) vector, $\mathbf{M}=\left\{M_{r} ; M_{\Theta} ; 0\right\}$ is magnetization vector (with $\mathbf{M}=0$ for the vacuum/iron or $\mathbf{M} \neq 0$ for the magnets according to the magnetization direction [43]), and $\mu=\mu_{0} \cdot \mu_{r}$ is the absolute magnetic permeability of the magnetic 
material in which $\mu_{0}$ and $\mu_{r}$ are respectively the vacuum permeability and the relative permeability of the magnetic material (with $\mu_{r}=1$ for the vacuum or $\mu_{r} \neq 1$ for the magnets/iron).

The magnetic vector potential $A_{z}$ is governed by Poisson's equation (i.e., $E S \neq 0$ ) or Laplace's equation (i.e., $E S=0$ ). Using the method of separation of variables, the 2-D general solution of $A_{z}$ in both directions (i.e., $r$ - and $\Theta$-edges) can be written as Fourier's series

$$
\begin{aligned}
& A_{z}=A_{z}^{\Theta}+A_{z}^{r}+A_{z P}, \\
& A_{z}^{\Theta}=\mid \begin{array}{l}
{\left[C_{0}^{\Theta}+D_{0}^{\Theta} \cdot \ln (r)\right] \cdot\left(E_{0}^{\Theta}+F_{0}^{\Theta} \cdot \Theta\right)} \\
\cdots+\sum_{h=1}^{\infty}\left(\begin{array}{l}
C_{h}^{\Theta} \cdot r^{\beta_{h}} \\
\cdots+D_{h}^{\Theta} \cdot r^{-\beta_{h}}
\end{array}\right) \cdot\left[\begin{array}{l}
E_{h}^{\Theta} \cdot \cos \left(\beta_{h} \cdot \Theta\right) \\
\cdots+F_{h}^{\Theta} \cdot \sin \left(\beta_{h} \cdot \Theta\right)
\end{array}\right],
\end{array}, \\
& \left.A_{z}^{r}=\mid \begin{array}{l}
{\left[C_{0}^{r}+D_{0}^{r} \cdot \ln (r)\right] \cdot\left(E_{0}^{r}+F_{0}^{r} \cdot \Theta\right)} \\
\cdots+\sum_{n=1}^{\infty}\left\{\begin{array}{l}
C_{n}^{r} \cdot \cos \left[\lambda_{n} \cdot \ln (r)\right] \\
\cdots+D_{n}^{r} \cdot \sin \left[\lambda_{n} \cdot \ln (r)\right]
\end{array}\right.
\end{array}\right\} \cdot\left[\begin{array}{l}
E_{n}^{r} \cdot \operatorname{ch}\left(\lambda_{n} \cdot \Theta\right) \\
\cdots+F_{n}^{r} \cdot \operatorname{sh}\left(\lambda_{n} \cdot \Theta\right)
\end{array}\right],
\end{aligned}
$$

where $A_{z P}$ is the particular solution of $A_{z}$ respecting the second member $E S$ in (A.1), $C_{0}^{\Theta}-F_{h}^{\Theta} \& C_{0}^{r}-F_{h}^{r}$ the integration constants, $\beta_{h} \& \lambda_{n}$ the spatial frequency (or periodicity) of $A_{z}^{\Theta} \& A_{z}^{r}$, and $h \& n$ the spatial harmonic orders.

Using $\mathbf{B}=\nabla \times \mathbf{A}$, the components of magnetic flux density $\mathbf{B}=\left\{B_{r} ; B_{\Theta} ; 0\right\}$ can be deduced by

$$
B_{r}=\frac{1}{r} \cdot \frac{\partial A_{z}}{\partial \Theta} \quad \text { and } \quad B_{\Theta}=-\frac{\partial A_{z}}{\partial r}
$$

which leads to

$$
\begin{aligned}
& B_{r}=B_{r}^{\Theta}+B_{r}^{r}+\frac{1}{r} \cdot \frac{\partial A_{z P}}{\partial \Theta}, \\
& B_{r}^{\Theta}=\mid \begin{array}{l}
\frac{F_{0}^{\Theta}}{r} \cdot\left[C_{0}^{\Theta}+D_{0}^{\Theta} \cdot \ln (r)\right] \\
\cdots+\sum_{h=1}^{\infty} \frac{\beta_{h}}{r} \cdot\left(\begin{array}{l}
C_{h}^{\Theta} \cdot r^{\beta_{h}} \\
\cdots+D_{h}^{\Theta} \cdot r^{-\beta_{h}}
\end{array}\right) \cdot\left[\begin{array}{l}
-E_{h}^{\Theta} \cdot \sin \left(\beta_{h} \cdot \Theta\right) \\
\cdots+F_{h}^{\Theta} \cdot \cos \left(\beta_{h} \cdot \Theta\right)
\end{array}\right],
\end{array} \\
& B_{r}^{r}=\mid \begin{array}{l}
\frac{F_{0}^{r}}{r} \cdot\left[C_{0}^{r}+D_{0}^{r} \cdot \ln (r)\right] \\
\cdots+\sum_{n=1}^{\infty} \frac{\lambda_{n}}{r} \cdot\left\{\begin{array}{l}
C_{n}^{r} \cdot \cos \left[\lambda_{n} \cdot \ln (r)\right] \\
\cdots+D_{n}^{r} \cdot \sin \left[\lambda_{n} \cdot \ln (r)\right]
\end{array}\right\} \cdot\left[\begin{array}{l}
E_{n}^{r} \cdot \operatorname{sh}\left(\lambda_{n} \cdot \Theta\right) \\
\cdots+F_{n}^{r} \cdot \operatorname{ch}\left(\lambda_{n} \cdot \Theta\right)
\end{array}\right],
\end{array}
\end{aligned}
$$

and

$$
\begin{aligned}
& B_{\Theta}=B_{\Theta}^{\Theta}+B_{\Theta}^{r}-\frac{\partial A_{z P}}{\partial r}, \\
& B_{\Theta}^{\Theta}=-\mid \begin{array}{l}
\frac{D_{0}^{\Theta}}{r} \cdot\left(E_{0}^{\Theta}+F_{0}^{\Theta} \cdot \Theta\right) \\
\cdots+\sum_{h=1}^{\infty} \frac{\beta_{h}}{r} \cdot\left(\begin{array}{l}
C_{h}^{\Theta} \cdot r^{\beta_{h}} \\
\cdots-D_{h}^{\Theta} \cdot r^{-\beta_{h}}
\end{array}\right) \cdot\left[\begin{array}{l}
E_{h}^{\Theta} \cdot \cos \left(\beta_{h} \cdot \Theta\right) \\
\cdots+F_{h}^{\Theta} \cdot \sin \left(\beta_{h} \cdot \Theta\right)
\end{array}\right],
\end{array}, \\
& \left.B_{\Theta}^{r}=-\mid \begin{array}{l}
\frac{D_{0}^{r}}{r} \cdot\left(E_{0}^{r}+F_{0}^{r} \cdot \Theta\right) \\
\cdots+\sum_{n=1}^{\infty} \frac{\lambda_{n}}{r} \cdot\left\{\begin{array}{l}
-C_{n}^{r} \cdot \sin \left[\lambda_{n} \cdot \ln (r)\right] \\
\cdots+D_{n}^{r} \cdot \cos \left[\lambda_{n} \cdot \ln (r)\right]
\end{array}\right.
\end{array}\right\} \cdot\left[\begin{array}{l}
E_{n}^{r} \cdot \operatorname{ch}\left(\lambda_{n} \cdot \Theta\right) \\
\cdots+F_{n}^{r} \cdot \operatorname{sh}\left(\lambda_{n} \cdot \Theta\right)
\end{array}\right] .
\end{aligned}
$$




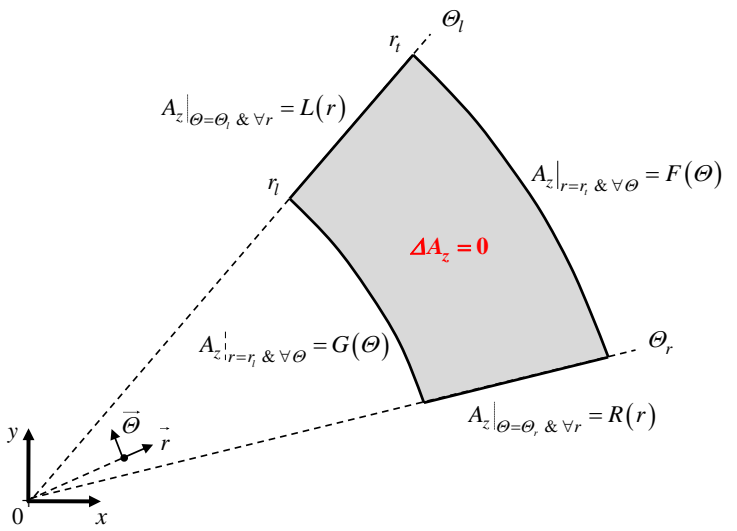

(a)

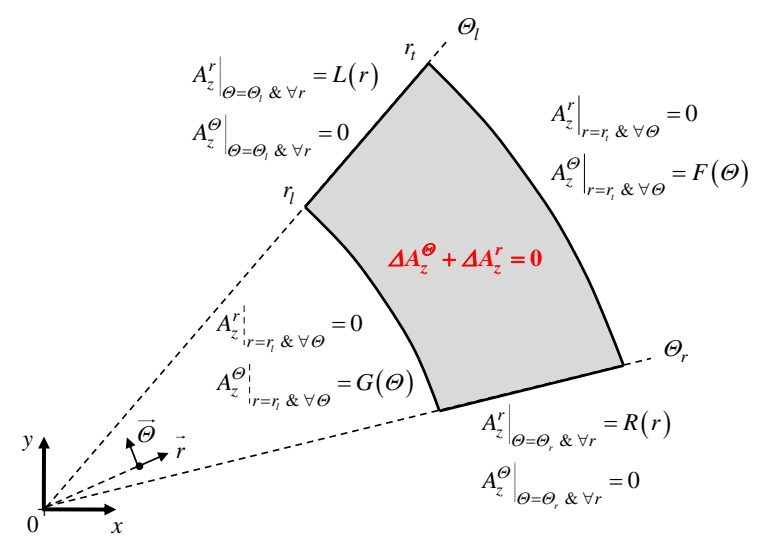

(b)

Figure B.1. $A_{z}$ imposed on all edges of a region: (a) General and (b) Principle of superposition.

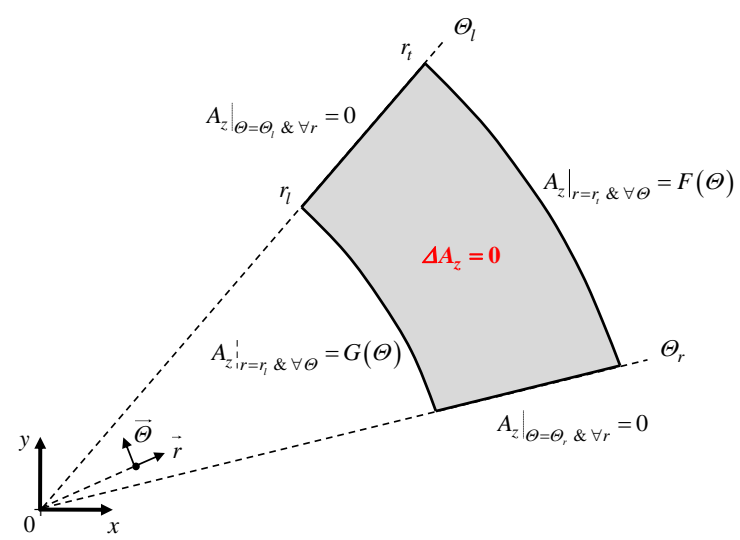

Figure B.2. Particular case: $A_{z}=0$ on $\Theta$-edges and $A_{z}$ imposed on $r$-edges of a region.

\section{Appendix B Simplification of Laplace's Equations according to imposed BCs}

Appendix B.1 Case-Study no 1: "A imposed on all edges of a region"

Figure B.1a shows a region (for $\Theta \in\left[\Theta_{r}, \Theta_{l}\right]$ and $r \in\left[r_{l}, r_{t}\right]$ ) whose $A_{z}$ imposed on all edges. By respecting the $\mathrm{BC}$ and applying the principle of superposition on the magnetic quantities, Figure B.1a is redefined by Figure B.1b.

In the case-study no $1, A_{z}=A_{z}^{\Theta}+A_{z}^{r}$, i.e., (A.2), is redefined by

$$
\begin{array}{r}
A_{z}^{\Theta}=\sum_{h=1}^{\infty}\left[c_{h}^{\Theta} \cdot r_{l} \cdot \frac{E_{\phi}\left(\beta_{h}, r_{t}, r\right)}{E_{\phi}\left(\beta_{h}, r_{t}, r_{l}\right)}+d_{h}^{\Theta} \cdot r_{t} \cdot \frac{E_{\phi}\left(\beta_{h}, r_{,} r_{l}\right)}{E_{\phi}\left(\beta_{h}, r_{t}, r_{l}\right)}\right] \cdot \sin \left[\beta_{h} \cdot\left(\Theta-\Theta_{r}\right)\right], \\
A_{z}^{r}=\sum_{n=1}^{\infty}\left\{e_{n}^{r} \cdot \frac{\operatorname{sh}\left[\lambda_{n} \cdot\left(\Theta_{l}-\Theta\right)\right]}{s h\left(\lambda_{n} \cdot \tau_{\Theta}\right)}+f_{n}^{r} \cdot \frac{s h\left[\lambda_{n} \cdot\left(\Theta-\Theta_{r}\right)\right]}{s h\left(\lambda_{n} \cdot \tau_{\Theta}\right)}\right\} \cdot r_{l} \cdot \sin \left[\lambda_{n} \cdot \ln \left(\frac{r}{r_{l}}\right)\right],
\end{array}
$$

the component $B_{r}=B_{r}^{\Theta}+B_{r}^{r}$ of $\mathbf{B}$, i.e., (A.4), by

$$
B_{r}^{\Theta}=\sum_{h=1}^{\infty} \beta_{h} \cdot\left[c_{h}^{\Theta} \cdot \frac{r_{l}}{r} \cdot \frac{E_{\phi}\left(\beta_{h}, r_{t}, r\right)}{E_{\phi}\left(\beta_{h}, r_{t}, r_{l}\right)}+d_{h}^{\Theta} \cdot \frac{r_{t}}{r} \cdot \frac{E_{\phi}\left(\beta_{h}, r_{,} r_{l}\right)}{E_{\phi}\left(\beta_{h}, r_{t}, r_{l}\right)}\right] \cdot \cos \left[\beta_{h} \cdot\left(\Theta-\Theta_{r}\right)\right],
$$




$$
B_{r}^{r}=\sum_{n=1}^{\infty} \lambda_{n} \cdot\left\{-e_{n}^{r} \cdot \frac{\operatorname{ch}\left[\lambda_{n} \cdot\left(\Theta_{l}-\Theta\right)\right]}{\operatorname{sh}\left(\lambda_{n} \cdot \tau_{\Theta}\right)}+f_{n}^{r} \cdot \frac{\operatorname{ch}\left[\lambda_{n} \cdot\left(\Theta-\Theta_{r}\right)\right]}{\operatorname{sh}\left(\lambda_{n} \cdot \tau_{\Theta}\right)}\right\} \cdot \frac{r_{l}}{r} \cdot \sin \left[\lambda_{n} \cdot \ln \left(\frac{r}{r_{l}}\right)\right],
$$

and the component $B_{\Theta}=B_{\Theta}^{\Theta}+B_{\Theta}^{r}$ of $\mathbf{B}$, i.e., (A.5), by

$$
\begin{gathered}
B_{\Theta}^{\Theta}=-\sum_{h=1}^{\infty} \beta_{h} \cdot\left[-c_{h}^{\Theta} \cdot \frac{r_{l}}{r} \cdot \frac{P_{\phi}\left(\beta_{h}, r_{t}, r\right)}{E_{\phi}\left(\beta_{h}, r_{t}, r_{l}\right)}+d_{h}^{\Theta} \cdot \frac{r_{t}}{r} \cdot \frac{P_{\phi}\left(\beta_{h}, r, r_{l}\right)}{E_{\phi}\left(\beta_{h}, r_{t}, r_{l}\right)}\right] \cdot \sin \left[\beta_{h} \cdot\left(\Theta-\Theta_{r}\right)\right], \\
B_{\Theta}^{r}=-\sum_{n=1}^{\infty} \lambda_{n} \cdot\left\{e_{n}^{r} \cdot \frac{\operatorname{sh}\left[\lambda_{n} \cdot\left(\Theta_{l}-\Theta\right)\right]}{\operatorname{sh}\left(\lambda_{n} \cdot \tau_{\Theta}\right)}+f_{n}^{r} \cdot \frac{\operatorname{sh}\left[\lambda_{n} \cdot\left(\Theta-\Theta_{r}\right)\right]}{s h\left(\lambda_{n} \cdot \tau_{\Theta}\right)}\right\} \cdot \frac{r_{l}}{r} \cdot \cos \left[\lambda_{n} \cdot \ln \left(\frac{r}{r_{l}}\right)\right],
\end{gathered}
$$

where $c_{h}^{\Theta}, d_{h}^{\Theta}, e_{n}^{r}$ and $f_{n}^{r}$ are new integration constants; $\beta_{h}=h \cdot \pi / \tau_{\Theta}$ with $\tau_{\Theta}=\Theta_{l}-\Theta_{r}$; $\lambda_{n}=n \cdot \pi / \tau_{r}$ with $\tau_{r}=\ln \left(r_{t} / r_{l}\right)$; and $E_{\phi}(w, x, y) \& P_{\phi}(w, x, y)$ are [44]

$$
E_{\phi}(w, x, y)=\left(\frac{x}{y}\right)^{w}-\left(\frac{y}{x}\right)^{w} \text { and } P_{\phi}(w, x, y)=\left(\frac{x}{y}\right)^{w}+\left(\frac{y}{x}\right)^{w},
$$

with

$$
\begin{array}{ll}
\frac{\partial E_{\phi}(w, x, y)}{\partial x}=\frac{w}{x} \cdot P_{\phi}(w, x, y) \quad \text { and } \quad & \frac{\partial E_{\phi}(w, x, y)}{\partial y}=-\frac{w}{y} \cdot P_{\phi}(w, x, y), \\
\frac{\partial P_{\phi}(w, x, y)}{\partial x}=\frac{w}{x} \cdot E_{\phi}(w, x, y) & \text { and } \quad \frac{\partial P_{\phi}(w, x, y)}{\partial y}=-\frac{w}{y} \cdot E_{\phi}(w, x, y) .
\end{array}
$$

When $A_{z}=0$ on $\Theta$-edges and $A_{z}$ imposed on $r$-edges (see Figure B.2), $A_{z}$ with $A_{z}^{r}=0$ in (B.1) is expressed by

$$
A_{z}=\sum_{h=1}^{\infty}\left[c_{h}^{\Theta} \cdot r_{l} \cdot \frac{E_{\phi \phi}\left(\beta_{h}, r_{t}, r\right)}{E_{\phi}\left(\beta_{h}, r_{t}, r_{l}\right)}+d_{h}^{\Theta} \cdot r_{t} \cdot \frac{E_{\phi}\left(\beta_{h}, r, r_{l}\right)}{E_{\phi}\left(\beta_{h}, r_{t}, r_{l}\right)}\right] \cdot \sin \left[\beta_{h} \cdot\left(\Theta-\Theta_{r}\right)\right],
$$

the $r$-component of $\mathbf{B}$ with $B_{r}^{r}=0$ in (B.2) by

$$
B_{r}=\sum_{h=1}^{\infty} \beta_{h} \cdot\left[c_{h}^{\Theta} \cdot \frac{r_{l}}{r} \cdot \frac{E_{\phi}\left(\beta_{h}, r_{t}, r\right)}{E_{\phi}\left(\beta_{h}, r_{t}, r_{l}\right)}+d_{h}^{\Theta} \cdot \frac{r_{t}}{r} \cdot \frac{E_{\phi}\left(\beta_{h}, r, r_{l}\right)}{E_{\phi}\left(\beta_{h}, r_{t}, r_{l}\right)}\right] \cdot \cos \left[\beta_{h} \cdot\left(\Theta-\Theta_{r}\right)\right],
$$

the $\Theta$-component of $\mathbf{B}$ with $B_{\Theta}^{r}=0$ in (B.3) by

$$
B_{\Theta}=-\sum_{h=1}^{\infty} \beta_{h} \cdot\left[-c_{h}^{\Theta} \cdot \frac{r_{l}}{r} \cdot \frac{P_{\phi}\left(\beta_{h}, r_{t}, r\right)}{E_{\phi}\left(\beta_{h}, r_{t}, r_{l}\right)}+d_{h}^{\Theta} \cdot \frac{r_{t}}{r} \cdot \frac{P_{\phi}\left(\beta_{h}, r, r_{l}\right)}{E_{\phi}\left(\beta_{h}, r_{t}, r_{l}\right)}\right] \cdot \sin \left[\beta_{h} \cdot\left(\Theta-\Theta_{r}\right)\right] .
$$

Appendix B.2 Case-Study no 2: " $B_{r}$ and $A_{z}$ are respectively imposed on $r$-and $\Theta$-edges of a region"

Figure B.3a shows a region (for $\Theta \in\left[\Theta_{r}, \Theta_{l}\right]$ and $r \in\left[r_{l}, r_{t}\right]$ ) whose $B_{r}$ and $A_{z}$ are respectively imposed on $r$ - and $\Theta$-edges. By respecting the BCs and applying the principle of superposition on the magnetic quantities, Figure B.3a is redefined by Figure B.3b.

In the case-study no $2, A_{z}=A_{z}^{\Theta}+A_{z}^{r}$, i.e., (A.2), is redefined by

$$
\begin{gathered}
A_{z}^{\Theta}=\mid \begin{array}{l}
c_{0}^{\Theta} \cdot r_{l} \cdot \frac{\ln \left(r_{t} / r\right)}{\ln \left(r_{t} / r_{l}\right)}+d_{0}^{\Theta} \cdot r_{t} \cdot \frac{\ln \left(r / r_{l}\right)}{\ln \left(r_{t} / r_{l}\right)} \\
\cdots+\sum_{h=1}^{\infty}\left[c_{h}^{\Theta} \cdot r_{l} \cdot \frac{E_{\phi}\left(\beta_{h}, r_{t}, r\right)}{E_{\phi}\left(\beta_{h}, r_{t}, r_{l}\right)}+d_{h}^{\Theta} \cdot r_{t} \cdot \frac{E_{\phi}\left(\beta_{h}, r, r_{l}\right)}{E_{\phi}\left(\beta_{h}, r_{t}, r_{l}\right)}\right] \cdot \cos \left[\beta_{h} \cdot\left(\Theta-\Theta_{r}\right)\right]
\end{array}, \\
A_{z}^{r}=\sum_{n=1}^{\infty}\left\{e_{n}^{r} \cdot \frac{\operatorname{ch}\left[\lambda_{n} \cdot\left(\Theta-\Theta_{r}\right)\right]}{\operatorname{sh}\left(\lambda_{n} \cdot \tau_{\Theta}\right)}-f_{n}^{r} \cdot \frac{\operatorname{ch}\left[\lambda_{n} \cdot\left(\Theta_{l}-\Theta\right)\right]}{\operatorname{sh}\left(\lambda_{n} \cdot \tau_{\Theta}\right)}\right\} \cdot \frac{r_{l}}{\lambda_{n}} \cdot \sin \left[\lambda_{n} \cdot \ln \left(\frac{r}{r_{l}}\right)\right],
\end{gathered}
$$




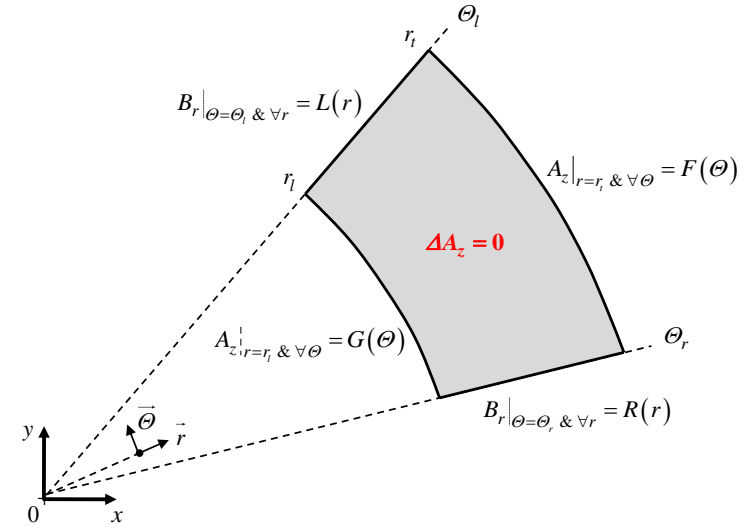

(a)

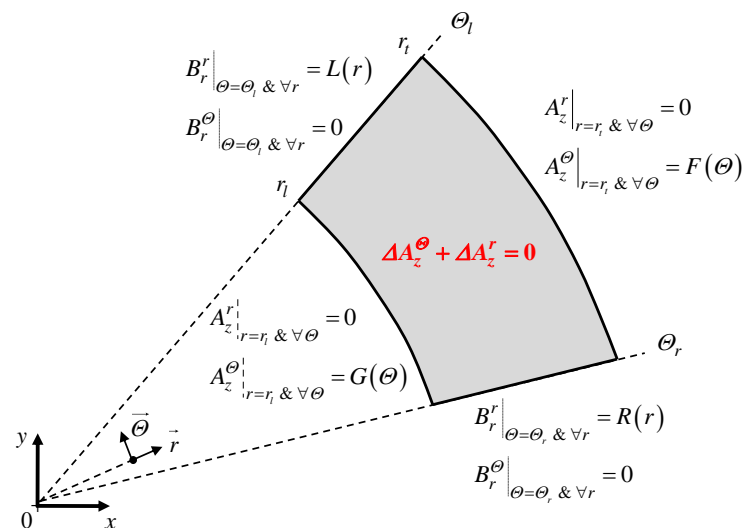

(b)

Figure B.3. $B_{r}$ imposed on $r$-edges and $A_{z}$ imposed on $\Theta$-edges of a region: (a) General and (b) Principle of superposition.

the component $B_{r}=B_{r}^{\Theta}+B_{r}^{r}$ of $\mathbf{B}$, i.e., (A.4), by

$$
\begin{aligned}
& B_{r}^{\Theta}=-\sum_{h=1}^{\infty} \beta_{h} \cdot\left[c_{h}^{\Theta} \cdot \frac{r_{l}}{r} \cdot \frac{E_{\phi}\left(\beta_{h}, r_{t}, r\right)}{E_{\phi}\left(\beta_{h}, r_{t}, r_{l}\right)}+d_{h}^{\Theta} \cdot \frac{r_{t}}{r} \cdot \frac{E_{\phi}\left(\beta_{h}, r, r_{l}\right)}{E_{\phi}\left(\beta_{h}, r_{t}, r_{l}\right)}\right] \cdot \sin \left[\beta_{h} \cdot\left(\Theta-\Theta_{r}\right)\right], \\
& B_{r}^{r}=\sum_{n=1}^{\infty}\left\{e_{n}^{r} \cdot \frac{\operatorname{sh}\left[\lambda_{n} \cdot\left(\Theta-\Theta_{r}\right)\right]}{\operatorname{sh}\left(\lambda_{n} \cdot \tau_{\Theta}\right)}+f_{n}^{r} \cdot \frac{\operatorname{sh}\left[\lambda_{n} \cdot\left(\Theta_{l}-\Theta\right)\right]}{\operatorname{sh}\left(\lambda_{n} \cdot \tau_{\Theta}\right)}\right\} \cdot \frac{r_{l}}{r} \cdot \sin \left[\lambda_{n} \cdot \ln \left(\frac{r}{r_{l}}\right)\right],
\end{aligned}
$$

and the component $B_{\Theta}=B_{\Theta}^{\Theta}+B_{\Theta}^{r}$ of $\mathbf{B}$, i.e., (A.5), by

$$
\begin{aligned}
& B_{\Theta}^{\Theta}=\mid \begin{array}{l}
c_{0}^{\Theta} \cdot \frac{r_{l}}{r} \cdot \frac{1}{\ln \left(r_{t} / r_{l}\right)}-d_{0}^{\Theta} \cdot \frac{r_{t}}{r} \cdot \frac{1}{\ln \left(r_{t} / r_{l}\right)} \\
\cdots+\sum_{h=1}^{\infty} \beta_{h} \cdot\left[c_{h}^{\Theta} \cdot \frac{r_{l}}{r} \cdot \frac{P_{\phi}\left(\beta_{h}, r_{t}, r\right)}{E_{\phi}\left(\beta_{h}, r_{t}, r_{l}\right)}-d_{h}^{\Theta} \cdot \frac{r_{t}}{r} \cdot \frac{P_{\phi}\left(\beta_{h}, r_{r}, r_{l}\right)}{E_{\phi}\left(\beta_{h}, r_{t}, r_{l}\right)}\right] \cdot \cos \left[\beta_{h} \cdot\left(\Theta-\Theta_{r}\right)\right]
\end{array}, \\
& B_{\Theta}^{r}=-\sum_{n=1}^{\infty}\left\{e_{n}^{r} \cdot \frac{\operatorname{ch}\left[\lambda_{n} \cdot\left(\Theta-\Theta_{r}\right)\right]}{\operatorname{sh}\left(\lambda_{n} \cdot \tau_{\Theta}\right)}-f_{n}^{r} \cdot \frac{\operatorname{ch}\left[\lambda_{n} \cdot\left(\Theta_{l}-\Theta\right)\right]}{\operatorname{sh}\left(\lambda_{n} \cdot \tau_{\Theta}\right)}\right\} \cdot \frac{r_{l}}{r} \cdot \cos \left[\lambda_{n} \cdot \ln \left(\frac{r}{r_{l}}\right)\right],
\end{aligned}
$$

where $c_{0}^{\Theta}, d_{0}^{\Theta}, c_{h}^{\Theta}, d_{h}^{\Theta}, e_{n}^{r}$ and $f_{n}^{r}$ are new integration constants.

\section{Appendix C Solving of Linear System}

\section{Appendix C.1 Calculation of General Integrals}

For the determination of the Fourier's series coefficients, it is required to calculate general integrals of the form

$$
\begin{gathered}
F_{1}^{\Theta}=\int_{l_{l}}^{l_{l}+w} \sin \left[\alpha_{s} \cdot\left(l-l_{s}\right)\right] \cdot d l, \\
F_{2}^{\Theta}=\int_{l_{l}}^{l_{l}+w} \cos \left[\alpha_{c} \cdot\left(l-l_{c}\right)\right] \cdot \sin \left[\alpha_{s} \cdot\left(l-l_{s}\right)\right] \cdot d l, \\
F_{3}^{\Theta}=\int_{l_{l}}^{l_{l}+w} \sin \left[\alpha_{s 1} \cdot\left(l-l_{s 1}\right)\right] \cdot \sin \left[\alpha_{s 2} \cdot\left(l-l_{s 2}\right)\right] \cdot d l,
\end{gathered}
$$




$$
\begin{aligned}
& F_{4}^{\Theta}=\int_{l_{l}}^{l_{l}+w} \operatorname{ch}\left[\alpha_{c h} \cdot\left(l-l_{c h}\right)\right] \cdot \sin \left[\alpha_{s} \cdot\left(l-l_{s}\right)\right] \cdot d l \\
& F_{5}^{\Theta}=\int_{l_{l}}^{l_{l}+w} \operatorname{sh}\left[\alpha_{s h} \cdot\left(l-l_{s h}\right)\right] \cdot \sin \left[\alpha_{s} \cdot\left(l-l_{s}\right)\right] \cdot d l \\
& F_{1}^{r}=\int_{r_{l}}^{r_{t}} \frac{1}{r} \cdot \sin \left[\alpha_{s 1} \cdot \ln \left(\frac{r}{r_{l}}\right)\right] \cdot \sin \left[\alpha_{s 2} \cdot \ln \left(\frac{r}{r_{l}}\right)\right] \cdot d r, \\
& F_{2}^{r}=\int_{r_{l}}^{r_{t}} r \cdot \sin \left[\alpha_{s} \cdot \ln \left(\frac{r}{r_{l}}\right)\right] \cdot d r \\
& F_{3}^{r}=\int_{r_{l}}^{r_{t}} \frac{1}{r} \cdot \frac{\ln \left(r_{t} / r\right)}{\ln \left(r_{t} / r_{l}\right)} \cdot \sin \left[\alpha_{s} \cdot \ln \left(\frac{r}{r_{l}}\right)\right] \cdot d r, \\
& F_{4}^{r}=\int_{r_{l}}^{r_{t}} \frac{1}{r} \cdot \frac{\ln \left(r / r_{l}\right)}{\ln \left(r_{t} / r_{l}\right)} \cdot \sin \left[\alpha_{s} \cdot \ln \left(\frac{r}{r_{l}}\right)\right] \cdot d r, \\
& F_{5}^{r}=\int_{r_{l}}^{r_{t}} \frac{1}{r} \cdot \frac{E_{\phi}\left(w, r_{t}, r\right)}{E_{\phi}\left(w, r_{t}, r_{l}\right)} \cdot \sin \left[\alpha_{s} \cdot \ln \left(\frac{r}{r_{l}}\right)\right] \cdot d r, \\
& F_{6}^{r}=\int_{r_{l}}^{r_{t}} \frac{1}{r} \cdot \frac{E_{\phi}\left(w, r, r_{l}\right)}{E_{\phi}\left(w, r_{t}, r_{l}\right)} \cdot \sin \left[\alpha_{s} \cdot \ln \left(\frac{r}{r_{l}}\right)\right] \cdot d r .
\end{aligned}
$$

The functions (C.1) will be used in the expression of the integration constants. The expressions of (C.1a) - (C.1e) have given in [1,44]. The development of (C.1f) - (C.1k) gives

$$
\begin{gathered}
F_{1}^{r}\left(\alpha_{s 1}, \alpha_{s 2}, r_{l}, r_{t}\right)=\frac{\ln \left(r_{t} / r_{l}\right)}{2} \cdot\left\{\operatorname{sinc}\left[\left(\alpha_{s 1}-\alpha_{s 2}\right) \cdot \ln \left(\frac{r_{t}}{r_{l}}\right)\right]-\operatorname{sinc}\left[\left(\alpha_{s 1}+\alpha_{s 2}\right) \cdot \ln \left(\frac{r_{t}}{r_{l}}\right)\right]\right\}, \\
F_{2}^{r}\left(\alpha_{s}, r_{l}, r_{t}\right)=r_{t}^{2} \cdot \frac{\alpha_{s}}{\alpha_{s}^{2}+4} \cdot\left\{2 \cdot \ln \left(\frac{r_{t}}{r_{l}}\right) \cdot \operatorname{sinc}\left[\alpha_{s} \cdot \ln \left(\frac{r_{t}}{r_{l}}\right)\right]+\left(\frac{r_{l}}{r_{t}}\right)^{2}-\cos \left[\alpha_{s} \cdot \ln \left(\frac{r_{t}}{r_{l}}\right)\right]\right\}, \\
F_{3}^{r}\left(\alpha_{s}, r_{l}, r_{t}\right)=\frac{1}{\alpha_{s}} \cdot\left\{1-\operatorname{sinc}\left[\alpha_{s} \cdot \ln \left(\frac{r_{t}}{r_{l}}\right)\right]\right\}, \\
F_{4}^{r}\left(\alpha_{s}, r_{l}, r_{t}\right)=\frac{1}{\alpha_{s}} \cdot\left\{\operatorname{sinc}\left[\alpha_{s} \cdot \ln \left(\frac{r_{t}}{r_{l}}\right)\right]-\cos \left[\alpha_{s} \cdot \ln \left(\frac{r_{t}}{r_{l}}\right)\right]\right\}, \\
F_{5}^{r}\left(\alpha_{s}, r_{l}, r_{t}\right)=-\frac{\alpha_{s}}{w^{2}+\alpha_{s}^{2}} \cdot\left\{w \cdot \frac{2}{E_{\phi}\left(w, r_{t}, r_{l}\right)} \cdot \ln \left(\frac{r_{t}}{r_{l}}\right) \cdot \operatorname{sinc}\left[\alpha_{s} \cdot \ln \left(\frac{r_{t}}{r_{l}}\right)\right]-1\right\}, \\
F_{6}^{r}\left(\alpha_{s}, r_{l}, r_{t}\right)=\frac{\alpha_{s}}{w^{2}+\alpha_{s}^{2}} \cdot\left\{w \cdot \ln \left(\frac{r_{t}}{r_{l}}\right) \cdot \frac{P_{\phi}\left(w, r_{t}, r_{l}\right)}{E_{\phi}\left(w, r_{t}, r_{l}\right)} \cdot \operatorname{sinc}\left[\alpha_{s} \cdot \ln \left(\frac{r_{t}}{r_{l}}\right)\right]-\cos \left[\alpha_{s} \cdot \ln \left(\frac{r_{t}}{r_{l}}\right)\right]\right\} .
\end{gathered}
$$

Appendix C.2 Determination of Integral Constants

The integration constants are determined by solving

$$
[I C]=[B C]^{-1} \cdot[E S] \quad \text { (i.e., Cramer's system) }
$$


which consists of

$$
X_{\max }=\left[\begin{array}{l}
H 1_{\max }+H 2_{\max }+2 \cdot H 3_{\max }+N 3_{\max }+2 \cdot H 4_{\max }+N 4_{\max } \\
\cdots+2 \cdot\left(H 5_{\max }+N 5_{\max }\right)+2 \cdot\left(H 6_{\max }+N 6_{\max }+1\right)+2 \cdot\left(H 7_{\max }+N 7_{\max }+1\right)
\end{array}\right]
$$

equations and unknowns [1], where $H 1_{\max }-H 7_{\max }$ (for the $\Theta$-edges) and $N 3_{\max }-N 7_{\max }$ (for the $r$-edges) are the maximal number of spatial harmonics in the various regions for the computation of $A_{z}$ and $\mathbf{B}=\left\{B_{r} ; B_{\Theta} ; 0\right\}$. To solve (C.3), a numerical matrix inversion is required for the calculation

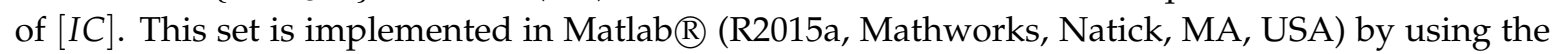
sparse matrix/vectors [1]. Usually, the two reasons for the possibility of including a finite number of harmonics is a limiting computational time and numerical accuracy [45].

The integration constants vector $[I C]$ (of dimension $X_{\max } \times 1$ ) is defined by

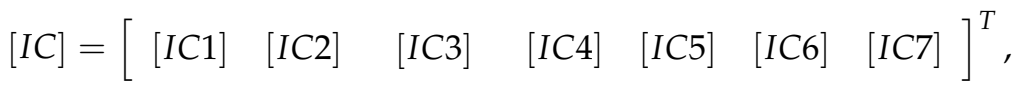

$$
\begin{aligned}
& {[I C 1]=\left[d 1_{h 1}^{\Theta}\right],} \\
& {[I C 2]=\left[c 2_{h 2}^{\Theta}\right] \text {, }} \\
& {[I C 3]=\left[\begin{array}{lll}
c 3_{h 3}^{\Theta} & d 3_{h 3}^{\Theta} & f 3_{n 3}^{r}
\end{array}\right],} \\
& {[I C 4]=\left[\begin{array}{lll}
c 4_{h 4}^{\Theta} & d 4_{h 4}^{\Theta} & e 4_{n 4}^{r}
\end{array}\right],} \\
& {[I C 5]=\left[\begin{array}{llll}
c 5_{h 5}^{\Theta} & d 5_{h 5}^{\Theta} & e 5_{n 5}^{r} & f 5_{n 5}^{r}
\end{array}\right],} \\
& {[I C 6]=\left[\begin{array}{llllll}
c 6_{0}^{\Theta} & c 6_{h 6}^{\Theta} & d 6_{0}^{\Theta} & d 6_{h 6}^{\Theta} & e 6_{n 6}^{r} & f 6_{n 6}^{r}
\end{array}\right],} \\
& {[I C 7]=\left[\begin{array}{llllll}
c 7_{0}^{\Theta} & c 7_{h 7}^{\Theta} & d 7_{0}^{\Theta} & d 7_{h 7}^{\Theta} & e 7_{n 7}^{r} & f 7_{n 7}^{r}
\end{array}\right] .}
\end{aligned}
$$

The structure of the electromagnetic sources vector $[E S]$ (of dimension $X_{\max } \times 1$ ) as well as the BCs matrix $[B C]$ (of dimension $X_{\max } \times X_{\max }$ ) is given in [1] (see $\S 2.6$. Solving of Linear System). The novel corresponding elements in $[E S]$ and $[B C]$ are defined as follows for Region 1

$$
\begin{gathered}
Q 13 c_{h 1, h 3}=-\frac{2 \cdot \beta 3_{h 3}}{\tau_{\Theta 1}} \cdot \frac{\mu_{1}}{\mu_{3}} \cdot \frac{P_{\phi}\left(\beta 3_{h 3}, r_{3}, r_{2}\right)}{E_{\phi}\left(\beta 3_{h 3}, r_{3}, r_{2}\right)} \cdot F_{3}^{\Theta}\left(\beta 3_{h 3}, \beta 1_{h 1}, \Theta_{1}, \Theta_{1}, \Theta_{1}, \tau_{\Theta 3}\right), \\
Q 13 d_{h 1, h 3}=\frac{2 \cdot \beta 3_{h 3}}{\tau_{\Theta 1}} \cdot \frac{\mu_{1}}{\mu_{3}} \cdot \frac{r_{3}}{r_{2}} \cdot \frac{2}{E_{\phi}\left(\beta 3_{h 3}, r_{3}, r_{2}\right)} \cdot F_{3}^{\Theta}\left(\beta 3_{h 3}, \beta 1_{h 1}, \Theta_{1}, \Theta_{1}, \Theta_{1}, \tau_{\Theta 3}\right), \\
Q 13 f_{h 1, n 3}=\frac{2 \cdot \lambda 3_{n 3}}{\tau_{\Theta 1}} \cdot \frac{\mu_{1}}{\mu_{3}} \cdot \operatorname{csch}\left(\lambda 3_{h 3} \cdot \tau_{\Theta 3}\right) \cdot F_{5}^{\Theta}\left(\lambda 3_{n 3}, \beta 1_{h 1}, \Theta_{1}, \Theta_{1}, \Theta_{1}, \tau_{\Theta 3}\right), \\
Q 14 c_{h 1, h 4}=-\frac{2 \cdot \beta 4_{h 4}}{\tau_{\Theta 1}} \cdot \frac{\mu_{1}}{\mu_{4}} \cdot \frac{P_{\phi}\left(\beta 4_{h 4}, r_{3}, r_{2}\right)}{E_{\phi}\left(\beta 4_{h 4}, r_{3}, r_{2}\right)} \cdot F_{3}^{\Theta}\left(\beta 4_{h 4}, \beta 1_{h 1}, \Theta_{5}, \Theta_{1}, \Theta_{5}, \tau_{\Theta 4}\right), \\
Q 14 d_{h 1, h 4}=\frac{2 \cdot \beta 4_{h 4}}{\tau_{\Theta 1}} \cdot \frac{\mu_{1}}{\mu_{4}} \cdot \frac{r_{3}}{r_{2}} \cdot \frac{2}{E_{\phi}\left(\beta 4_{h 4}, r_{3}, r_{2}\right)} \cdot F_{3}^{\Theta}\left(\beta 4_{h 4}, \beta 1_{h 1}, \Theta_{5}, \Theta_{1}, \Theta_{5}, \tau_{\Theta 4}\right), \\
Q 14 e_{h 1, n 4}=-\frac{2 \cdot \lambda 4_{n 4}}{\tau_{\Theta 1}} \cdot \frac{\mu_{1}}{\mu_{4}} \cdot c s c h\left(\lambda 4_{n 4} \cdot \tau_{\Theta 4}\right) \cdot F_{5}^{\Theta}\left(\lambda 4_{n 4}, \beta 1_{h 1}, \Theta_{6}, \Theta_{1}, \Theta_{5}, \tau_{\Theta 4}\right), \\
Q 15 c_{h 1, h 5}=-\frac{2 \cdot \beta 5_{h 5}}{\tau_{\Theta 1}} \cdot \frac{\mu_{1}}{\mu_{5}} \cdot \frac{P_{\phi}\left(\beta 5_{h 5}, r_{3}, r_{2}\right)}{E_{\phi}\left(\beta 5_{h 5}, r_{3}, r_{2}\right)} \cdot F_{3}^{\Theta}\left(\beta 5_{h 5}, \beta 1_{h 1}, \Theta_{3}, \Theta_{1}, \Theta_{3}, \tau_{\Theta 5}\right), \\
Q 15 d_{h 1, h 5}=\frac{2 \cdot \beta 5_{h 5}}{\tau_{\Theta 1}} \cdot \frac{\mu_{1}}{\mu_{5}} \cdot \frac{r_{3}}{r_{2}} \cdot \frac{2}{E_{\phi}\left(\beta 5_{h 5}, r_{3}, r_{2}\right)} \cdot F_{3}^{\Theta}\left(\beta 5_{h 5}, \beta 1_{h 1}, \Theta_{3}, \Theta_{1}, \Theta_{3}, \tau_{\Theta 5}\right),
\end{gathered}
$$




$$
\begin{aligned}
& Q 15 e_{h 1, n 5}=-\frac{2 \cdot \lambda 5_{n 5}}{\tau_{\Theta 1}} \cdot \frac{\mu_{1}}{\mu_{5}} \cdot \operatorname{csch}\left(\lambda 5_{n 5} \cdot \tau_{\Theta 5}\right) \cdot F_{5} \Theta\left(\lambda 5_{n 5}, \beta 1_{h 1}, \Theta_{4}, \Theta_{1}, \Theta_{3}, \tau_{\Theta 5}\right), \\
& Q 15 f_{h 1, n 5}=\frac{2 \cdot \lambda 5_{n 5}}{\tau_{\Theta 1}} \cdot \frac{\mu_{1}}{\mu_{5}} \cdot \operatorname{csch}\left(\lambda 5_{n 5} \cdot \tau_{\Theta 5}\right) \cdot F_{5}^{\Theta}\left(\lambda 5_{n 5}, \beta 1_{h 1}, \Theta_{3}, \Theta_{1}, \Theta_{3}, \tau_{\Theta 5}\right) \text {, } \\
& Q 16 c_{h 1, h 6}=-\frac{2}{\tau_{\Theta 1}} \cdot \frac{\mu_{1}}{\mu_{6}} \cdot \begin{cases}\frac{1}{\ln \left(r_{3} / r_{2}\right)} \cdot F_{1}^{\Theta}\left(\beta 1_{h 1}, \Theta_{1}, \Theta_{2}, \tau_{\Theta 6}\right) & \text { for } h 6=0 \\
\beta 6_{h 6} \cdot \frac{P_{\phi}\left(\beta 6_{h 6}, r_{3}, r_{2}\right)}{E_{\phi}\left(\beta 6_{h 6}, r_{3}, r_{2}\right)} \cdot F_{2}^{\Theta}\left(\beta 6_{h 6}, \beta 1_{h 1}, \Theta_{2}, \Theta_{1}, \Theta_{2}, \tau_{\Theta 6}\right) & \text { for } h 6 \neq 0\end{cases} \\
& Q 16 d_{h 1, h 6}=\frac{2}{\tau_{\Theta 1}} \cdot \frac{\mu_{1}}{\mu_{6}} \cdot \frac{r_{3}}{r_{2}} \cdot \begin{cases}\frac{1}{\ln \left(r_{3} / r_{2}\right)} \cdot F_{1}^{\Theta}\left(\beta 1_{h 1}, \Theta_{1}, \Theta_{2}, \tau_{\Theta 6}\right) & \text { for } h 6=0 \\
\frac{2 \cdot \beta 6_{h 6}}{E_{\phi}\left(\beta 6_{h 6}, r_{3}, r_{2}\right)} \cdot F_{2}^{\Theta}\left(\beta 6_{h 6}, \beta 1_{h 1}, \Theta_{2}, \Theta_{1}, \Theta_{2}, \tau_{\Theta 6}\right) & \text { for } h 6 \neq 0\end{cases} \\
& Q 16 e_{h 1, n 6}=\frac{2}{\tau_{\Theta 1}} \cdot \frac{\mu_{1}}{\mu_{6}} \cdot \operatorname{csch}\left(\lambda 6_{n 6} \cdot \tau_{\Theta 6}\right) \cdot F_{4}^{\Theta}\left(\lambda 6_{n 6}, \beta 1_{h 1}, \Theta_{2}, \Theta_{1}, \Theta_{2}, \tau_{\Theta 6}\right), \\
& Q 16 f_{h 1, n 6}=-\frac{2}{\tau_{\Theta 1}} \cdot \frac{\mu_{1}}{\mu_{6}} \cdot \operatorname{csch}\left(\lambda 6_{n 6} \cdot \tau_{\Theta 6}\right) \cdot F_{4}^{\Theta}\left(\lambda 6_{n 6}, \beta 1_{h 1}, \Theta_{3}, \Theta_{1}, \Theta_{2}, \tau_{\Theta 6}\right), \\
& Q 17 c_{h 1, h 7}=-\frac{2}{\tau_{\Theta 1}} \cdot \frac{\mu_{1}}{\mu_{7}} \cdot \begin{cases}\frac{1}{\ln \left(r_{3} / r_{2}\right)} \cdot F_{1}^{\Theta}\left(\beta 1_{h 1}, \Theta_{1}, \Theta_{4}, \tau_{\Theta 7}\right) & \text { for } h 7=0 \\
\beta 7_{h 7} \cdot \frac{P_{\phi}\left(\beta 7_{h 7}, r_{3}, r_{2}\right)}{E_{\phi}\left(\beta 7_{h 7}, r_{3}, r_{2}\right)} \cdot F_{2}^{\Theta}\left(\beta 7_{h 7}, \beta 1_{h 1}, \Theta_{4}, \Theta_{1}, \Theta_{4}, \tau_{\Theta 7}\right) & \text { for } h 7 \neq 0\end{cases} \\
& Q 17 d_{h 1, h 7}=\frac{2}{\tau_{\Theta 1}} \cdot \frac{\mu_{1}}{\mu_{7}} \cdot \frac{r_{3}}{r_{2}} \cdot \begin{cases}\frac{1}{\ln \left(r_{3} / r_{2}\right)} \cdot F_{1}^{\Theta}\left(\beta 1_{h 1}, \Theta_{1}, \Theta_{4}, \tau_{\Theta 7}\right) & \text { for } h 7=0 \\
\frac{2 \cdot \beta 7_{h 7}}{E_{\phi}\left(\beta 7_{h 7}, r_{3}, r_{2}\right)} \cdot F_{2}^{\Theta}\left(\beta 7_{h 7}, \beta 1_{h 1}, \Theta_{4}, \Theta_{1}, \Theta_{4}, \tau_{\Theta 7}\right) & \text { for } h 7 \neq 0\end{cases} \\
& Q 17 e_{h 1, n 7}=\frac{2}{\tau_{\Theta 1}} \cdot \frac{\mu_{1}}{\mu_{7}} \cdot \operatorname{csch}\left(\lambda 7_{n 7} \cdot \tau_{\Theta 7}\right) \cdot F_{4}^{\Theta}\left(\lambda 7_{n 7}, \beta 1_{h 1}, \Theta_{4}, \Theta_{1}, \Theta_{4}, \tau_{\Theta 7}\right) \text {, } \\
& Q 17 f_{h 1, n 7}=-\frac{2}{\tau_{\Theta 1}} \cdot \frac{\mu_{1}}{\mu_{7}} \cdot \operatorname{csch}\left(\lambda 7_{n 7} \cdot \tau_{\Theta 7}\right) \cdot F_{4}^{\Theta}\left(\lambda 7_{n 7}, \beta 1_{h 1}, \Theta_{5}, \Theta_{1}, \Theta_{4}, \tau_{\Theta 7}\right), \\
& E S 16_{h 1}+E S 17_{h 1}=\mu_{1} \cdot \frac{r_{2}}{\tau_{\Theta 1}} \cdot\left[J_{z 6} \cdot F_{1}^{\Theta}\left(\beta 1_{h 1}, \Theta_{1}, \Theta_{2}, \tau_{\Theta 6}\right)+J_{z 7} \cdot r_{2} \cdot F_{1}^{\Theta}\left(\beta 1_{h 1}, \Theta_{1}, \Theta_{4}, \tau_{\Theta 7}\right)\right],
\end{aligned}
$$
for Region 2

$$
\begin{gathered}
Q 23 c_{h 2, h 3}=-\frac{2 \cdot \beta 3_{h 3}}{\tau_{\Theta 2}} \cdot \frac{\mu_{2}}{\mu_{3}} \cdot \frac{r_{2}}{r_{3}} \cdot \frac{2}{E_{\phi}\left(\beta 3_{h 3}, r_{3}, r_{2}\right)} \cdot F_{3}^{\Theta}\left(\beta 3_{h 3}, \beta 2_{h 2}, \Theta_{1}, \Theta_{1}, \Theta_{1}, \tau_{\Theta 3}\right), \\
Q 23 d_{h 2, h 3}=\frac{2 \cdot \beta 3_{h 3}}{\tau_{x 2}} \cdot \frac{\mu_{2}}{\mu_{3}} \cdot \frac{P_{\phi}\left(\beta 3_{h 3}, r_{3}, r_{2}\right)}{E_{\phi}\left(\beta 3_{h 3}, r_{3}, r_{2}\right)} \cdot F_{3}^{\Theta}\left(\beta 3_{h 3}, \beta 2_{h 2}, \Theta_{1}, \Theta_{1}, \Theta_{1}, \tau_{\Theta 3}\right), \\
Q 23 f_{h 2, n 3}=\frac{2 \cdot \lambda 3_{n 3}}{\tau_{\Theta 2}} \cdot \frac{\mu_{2}}{\mu_{3}} \cdot \frac{r_{2}}{r_{3}} \cdot(-1)^{n 3} \cdot \operatorname{csch}\left(\lambda 3_{h 3} \cdot \tau_{\Theta 3}\right) \cdot F_{5}^{\Theta}\left(\lambda 3_{n 3}, \beta 2_{h 2}, \Theta_{1}, \Theta_{1}, \Theta_{1}, \tau_{\Theta 3}\right), \\
Q 24 c_{h 2, h 4}=-\frac{2 \cdot \beta 4_{h 4}}{\tau_{\Theta 2}} \cdot \frac{\mu_{2}}{\mu_{4}} \cdot \frac{r_{2}}{r_{3}} \cdot \frac{2}{E_{\phi}\left(\beta 4_{h 4}, r_{3}, r_{2}\right)} \cdot F_{3}^{\Theta}\left(\beta 4_{h 4}, \beta 2_{h 2}, \Theta_{5}, \Theta_{1}, \Theta_{5}, \tau_{\Theta 4}\right), \\
Q 24 d_{h 2, h 4}=\frac{2 \cdot \beta 4_{h 4}}{\tau_{x 2}} \cdot \frac{\mu_{2}}{\mu_{4}} \cdot \frac{P_{\phi}\left(\beta 4_{h 4}, r_{3}, r_{2}\right)}{E_{\phi}\left(\beta 4_{h 4}, r_{3}, r_{2}\right)} \cdot F_{3}^{\Theta}\left(\beta 4_{h 4}, \beta 2_{h 2}, \Theta_{5}, \Theta_{1}, \Theta_{5}, \tau_{\Theta 4}\right), \\
Q 2 e_{h 2, n 4}=-\frac{2 \cdot \lambda 4_{n 4}}{\tau_{\Theta 2}} \cdot \frac{\mu_{2}}{\mu_{4}} \cdot \frac{r_{2}}{r_{3}} \cdot(-1)^{n 4} \cdot c s c h\left(\lambda 4_{h 4} \cdot \tau_{\Theta 4}\right) \cdot F_{5}^{\Theta}\left(\lambda 4_{n 4}, \beta 2_{h 2}, \Theta_{6}, \Theta_{1}, \Theta_{5}, \tau_{\Theta 4}\right), \\
Q 25 c_{h 2, h 5}=-\frac{2 \cdot \beta 5_{h 5}}{\tau_{\Theta 2}} \cdot \frac{\mu_{2}}{\mu_{5}} \cdot \frac{r_{2}}{r_{3}} \cdot \frac{2}{E_{\phi}\left(\beta 5_{h 5}, r_{3}, r_{2}\right)} \cdot F_{3}^{\Theta}\left(\beta 5_{h 5}, \beta 2_{h 2}, \Theta_{3}, \Theta_{1}, \Theta_{3}, \tau_{\Theta 5}\right), \\
Q 25 d_{h 2, h 5}=\frac{2 \cdot \beta 5_{h 5}}{\tau_{\Theta 2}} \cdot \frac{\mu_{2}}{\mu_{5}} \cdot \frac{P_{\phi}\left(\beta 5_{h 5}, r_{3}, r_{2}\right)}{E_{\phi}\left(\beta 5_{h 5}, r_{3}, r_{2}\right)} \cdot F_{3}^{\Theta}\left(\beta 5_{h 5}, \beta 2_{h 2}, \Theta_{3}, \Theta_{1}, \Theta_{3}, \tau_{\Theta 5}\right),
\end{gathered}
$$




$$
\begin{aligned}
& Q 25 e_{h 2, n 5}=-\frac{2 \cdot \lambda 5_{n 5}}{\tau_{\Theta 2}} \cdot \frac{\mu_{2}}{\mu_{5}} \cdot \frac{r_{2}}{r_{3}} \cdot(-1)^{n 5} \cdot \operatorname{csch}\left(\lambda 5_{h 5} \cdot \tau_{\Theta 5}\right) \cdot F_{5}^{\Theta}\left(\lambda 5_{n 5}, \beta 2_{h 2}, \Theta_{4}, \Theta_{1}, \Theta_{3}, \tau_{\Theta 5}\right), \\
& Q 25 f_{h 2, n 5}=\frac{2 \cdot \lambda 5_{n 5}}{\tau_{\Theta 2}} \cdot \frac{\mu_{2}}{\mu_{5}} \cdot \frac{r_{2}}{r_{3}} \cdot(-1)^{n 5} \cdot \operatorname{csch}\left(\lambda 5_{h 5} \cdot \tau_{\Theta 5}\right) \cdot F_{5}^{\Theta}\left(\lambda 5_{n 5}, \beta 2_{h 2}, \Theta_{3}, \Theta_{1}, \Theta_{3}, \tau_{\Theta 5}\right) \text {, } \\
& Q 26 c_{h 2, h 6}=-\frac{2}{\tau_{\Theta 2}} \cdot \frac{\mu_{2}}{\mu_{6}} \cdot \frac{r_{2}}{r_{3}} \cdot \begin{cases}\frac{1}{\ln \left(r_{3} / r_{2}\right)} \cdot F_{1}^{\Theta}\left(\beta 2_{h 2}, \Theta_{1}, \Theta_{2}, \tau_{\Theta 6}\right) & \text { for } h 6=0 \\
\frac{2 \cdot \beta 6_{h 6}}{E_{\phi}\left(\beta 6_{h 6}, r_{3}, r_{2}\right)} \cdot F_{2}^{\Theta}\left(\beta 6_{h 6}, \beta 2 h 2, \Theta_{2}, \Theta_{1}, \Theta_{2}, \tau_{\Theta 6}\right) & \text { for } h 6 \neq 0\end{cases} \\
& Q 26 d_{h 2, h 6}=\frac{2}{\tau_{\Theta 2}} \cdot \frac{\mu_{2}}{\mu_{6}} \cdot \begin{cases}\frac{1}{\ln \left(r_{3} / r_{2}\right)} \cdot F_{1}^{\Theta}\left(\beta 2_{h 2}, \Theta_{1}, \Theta_{2}, \tau_{\Theta 6}\right) & \text { for } h 6=0 \\
\beta 6_{h 6} \cdot \frac{P_{\phi}\left(\beta 6_{h 6}, r_{3}, r_{2}\right)}{E_{\phi}\left(\beta 6_{h 6}, r_{3}, r_{2}\right)} \cdot F_{2}^{\Theta}\left(\beta 6_{h 6}, \beta 2_{h 2}, \Theta_{2}, \Theta_{1}, \Theta_{2}, \tau_{\Theta 6}\right) & \text { for } h 6 \neq 0\end{cases} \\
& Q 26 e_{h 2, n 6}=\frac{2}{\tau_{\Theta 2}} \cdot \frac{\mu_{2}}{\mu_{6}} \cdot \frac{r_{2}}{r_{3}} \cdot(-1)^{n 6} \cdot \operatorname{csch}\left(\lambda 6_{n 6} \cdot \tau_{\Theta 6}\right) \cdot F_{4}^{\Theta}\left(\lambda 6_{n 6}, \beta 2_{h 2}, \Theta_{2}, \Theta_{1}, \Theta_{2}, \tau_{\Theta 6}\right), \\
& Q 26 f_{h 2, n 6}=-\frac{2}{\tau_{\Theta 2}} \cdot \frac{\mu_{2}}{\mu_{6}} \cdot \frac{r_{2}}{r_{3}} \cdot(-1)^{n 6} \cdot \operatorname{csch}\left(\lambda 6_{n 6} \cdot \tau_{\Theta 6}\right) \cdot F_{4}^{\Theta}\left(\lambda 6_{n 6}, \beta 2_{h 2}, \Theta_{3}, \Theta_{1}, \Theta_{2}, \tau_{\Theta 6}\right) \text {, } \\
& Q 27 c_{h 2, h 7}=-\frac{2}{\tau_{\Theta 2}} \cdot \frac{\mu_{2}}{\mu_{7}} \cdot \frac{r_{2}}{r_{3}} \cdot \begin{cases}\frac{1}{\ln \left(r_{3} / r_{2}\right)} \cdot F_{1}^{\Theta}\left(\beta 2_{h 2}, \Theta_{1}, \Theta_{4}, \tau_{\Theta 7}\right) & \text { for } h 7=0 \\
\frac{2 \cdot \beta 7_{h 7}}{E_{\phi}\left(\beta 7_{h 7}, r_{3}, r_{2}\right)} \cdot F_{2}^{\Theta}\left(\beta 7_{h 7}, \beta 2_{h 2}, \Theta_{4}, \Theta_{1}, \Theta_{4}, \tau_{\Theta 7}\right) & \text { for } h 7 \neq 0\end{cases} \\
& Q 27 d_{h 2, h 7}=\frac{2}{\tau_{\Theta 2}} \cdot \frac{\mu_{2}}{\mu_{7}} \cdot \begin{cases}\frac{1}{\ln \left(r_{3} / r_{2}\right)} \cdot F_{1}^{\Theta}\left(\beta 2_{h 2}, \Theta_{1}, \Theta_{2}, \tau_{\Theta 7}\right) & \text { for } h 7=0 \\
\beta 7_{h 7} \cdot \frac{P_{\phi}\left(\beta 7_{h 7}, r_{3}, r_{2}\right)}{E_{\phi}\left(\beta 7_{h 7}, r_{3}, r_{2}\right)} \cdot F_{2}^{\Theta}\left(\beta 7_{h 7}, \beta 2_{h 2}, \Theta_{4}, \Theta_{1}, \Theta_{4}, \tau_{\Theta 7}\right) & \text { for } h 7 \neq 0\end{cases} \\
& Q 27 e_{h 2, n 7}=\frac{2}{\tau_{\Theta 2}} \cdot \frac{\mu_{2}}{\mu_{7}} \cdot \frac{r_{2}}{r_{3}} \cdot(-1)^{n 7} \cdot \operatorname{csch}\left(\lambda_{n 7} \cdot \tau_{\Theta 7}\right) \cdot F_{4}^{\Theta}\left(\lambda 7_{n 7}, \beta 2_{h 2}, \Theta_{4}, \Theta_{1}, \Theta_{4}, \tau_{\Theta 7}\right), \\
& Q 27 f_{h 2, n 7}=-\frac{2}{\tau_{\Theta 2}} \cdot \frac{\mu_{2}}{\mu_{7}} \cdot \frac{r_{2}}{r_{3}} \cdot(-1)^{n 7} \cdot \operatorname{csch}\left(\lambda_{n 7} \cdot \tau_{\Theta 7}\right) \cdot F_{4}^{\Theta}\left(\lambda 7_{n 7}, \beta 2_{h 2}, \Theta_{5}, \Theta_{1}, \Theta_{4}, \tau_{\Theta 7}\right) \text {, } \\
& E S 26_{h 2}+E S 27_{h 2}=\mu_{2} \cdot \frac{r_{3}}{\tau_{\Theta 2}} \cdot\left[J_{z 6} \cdot F_{1}^{\Theta}\left(\beta 2_{h 2}, \Theta_{1}, \Theta_{2}, \tau_{\Theta 6}\right)+J_{z 7} \cdot F_{1}^{\Theta}\left(\beta 2_{h 2}, \Theta_{1}, \Theta_{4}, \tau_{\Theta 7}\right)\right]
\end{aligned}
$$

for Region 3

$$
\begin{gathered}
Q 31 d_{h 3, h 1}=\frac{2}{\tau_{\Theta 3}} \cdot \frac{1}{\beta 1_{h 1}} \cdot \frac{E_{\phi}\left(\beta 1_{h 1}, r_{2}, r_{1}\right)}{P_{\phi}\left(\beta 1_{h 1}, r_{2}, r_{1}\right)} \cdot F_{3}^{\Theta}\left(\beta 1_{h 1}, \beta 3_{h 3}, \Theta_{1}, \Theta_{1}, \Theta_{1}, \tau_{\Theta 3}\right), \\
Q 32 c_{h 3, h 2}=-\frac{2}{\tau_{\Theta 3}} \cdot \frac{1}{\beta 2_{h 2}} \cdot \frac{E_{\phi}\left(\beta 2_{h 2}, r_{4}, r_{3}\right)}{P_{\phi}\left(\beta 2_{h 2}, r_{4}, r_{3}\right)} \cdot F_{3}^{\Theta}\left(\beta 2_{h 2}, \beta 3_{h 3}, \Theta_{1}, \Theta_{1}, \Theta_{1}, \tau_{\Theta 3}\right), \\
Q 36 c_{n 3, h 6}=-\frac{2}{\tau_{r 3}} \cdot \begin{cases}F_{3}^{r}\left(\lambda 3_{n 3}, r_{2}, r_{3}\right) & \text { for } h 6=0 \\
F_{5}^{r}\left(\beta 6_{h 6}, \lambda 3_{n 3}, r_{2}, r_{3}\right) & \text { for } h 6 \neq 0\end{cases} \\
Q 36 d_{n 3, h 6}=-\frac{2}{\tau_{r 3}} \cdot \frac{r_{3}}{r_{2}} \cdot \begin{cases}F_{4}^{r}\left(\lambda 3_{n 3}, r_{2}, r_{3}\right) & \text { for } h 6=0 \\
F_{6}^{r}\left(\beta 6_{h 6}, \lambda 3_{n 3}, r_{2}, r_{3}\right) & \text { for } h 6 \neq 0\end{cases} \\
Q 36 e_{n 3, n 6}=-\frac{2}{\tau_{r 3}} \cdot \frac{1}{\lambda 6_{n 6}} \cdot \operatorname{csch}\left(\lambda 6_{n 6} \cdot \tau_{\Theta 6}\right) \cdot F_{1}^{r}\left(\lambda 6_{n 6}, \lambda 3_{n 3}, r_{2}, r_{3}\right), \\
Q 36 f_{n 3, n 6}=\frac{2}{\tau_{r 3}} \cdot \frac{1}{\lambda 6_{n 6}} \cdot \operatorname{coth}\left(\lambda 6_{n 6} \cdot \tau_{\Theta 6}\right) \cdot F_{1}^{r}\left(\lambda 6_{n 6}, \lambda 3_{n 3}, r_{2}, r_{3}\right), \\
E S 36_{n 3}=-\mu_{6} \cdot \frac{1}{2 \cdot \tau_{r 3}} \cdot \frac{1}{r_{2}} \cdot J_{z 6} \cdot F_{2}^{r}\left(\lambda 3_{n 3}, r_{2}, r_{3}\right),
\end{gathered}
$$


for Region 4

$$
\begin{gathered}
Q 41 d_{h 4, h 1}=\frac{2}{\tau_{\Theta 4}} \cdot \frac{1}{\beta 1_{h 1}} \cdot \frac{E_{\phi}\left(\beta 1_{h 1}, r_{2}, r_{1}\right)}{P_{\phi}\left(\beta 1_{h 1}, r_{2}, r_{1}\right)} \cdot F_{3}^{\Theta}\left(\beta 1_{h 1}, \beta 4_{h 4}, \Theta_{1}, \Theta_{5}, \Theta_{5}, \tau_{\Theta 4}\right), \\
Q 42 c_{h 4, h 2}=-\frac{2}{\tau_{\Theta 4}} \cdot \frac{1}{\beta 2_{h 2}} \cdot \frac{E_{\phi}\left(\beta 2_{h 2}, r_{4}, r_{3}\right)}{P_{\phi}\left(\beta 2_{h 2}, r_{4}, r_{3}\right)} \cdot F_{3}^{\Theta}\left(\beta 2_{h 2}, \beta 4_{h 4}, \Theta_{1}, \Theta_{5}, \Theta_{5}, \tau_{\Theta 4}\right), \\
Q 47 c_{n 4, h 7}=-\frac{2}{\tau_{r 4}} \cdot \begin{cases}F_{3}^{r}\left(\lambda 4_{n 4}, r_{2}, r_{3}\right) & \text { for } h 7=0 \\
(-1)^{h 7} \cdot F_{5}^{r}\left(\beta 7_{h 7}, \lambda 4_{n 4}, r_{2}, r_{3}\right) & \text { for } h 7 \neq 0\end{cases} \\
Q 47 d_{n 4, h 7}=-\frac{2}{\tau_{r 4}} \cdot \frac{r_{3}}{r_{2}} \cdot \begin{cases}F_{4}^{r}\left(\lambda 4_{n 4}, r_{2}, r_{3}\right) & \text { for } h 7=0 \\
(-1)^{h 7} \cdot F_{6}^{r}\left(\beta 7_{h 7}, \lambda 4_{n 4}, r_{2}, r_{3}\right) & \text { for } h 7 \neq 0\end{cases} \\
Q 47 e_{n 4, n 7}=-\frac{2}{\tau_{r 4}} \cdot \frac{1}{\lambda 7_{n 7}} \cdot \operatorname{coth}\left(\lambda 7_{n 7} \cdot \tau_{\Theta 7}\right) \cdot F_{1}^{r}\left(\lambda 7_{n 7}, \lambda 4_{n 4}, r_{2}, r_{3}\right), \\
Q 47 f_{n 4, n 7}=\frac{2}{\tau_{r 4}} \cdot \frac{1}{\lambda 7_{n 7}} \cdot \operatorname{csch}\left(\lambda 7_{n 7} \cdot \tau_{\Theta 7}\right) \cdot F_{1}^{r}\left(\lambda 7_{n 7}, \lambda 4_{n 4}, r_{2}, r_{3}\right), \\
E S 47_{n 4}=-\mu_{7} \cdot \frac{1}{2 \cdot \tau_{r 4}} \cdot \frac{1}{r_{2}} \cdot J_{z 7} \cdot F_{2}^{r}\left(\lambda 4_{n 4}, r_{2}, r_{3}\right),
\end{gathered}
$$

for Region 5

$$
\begin{aligned}
& Q 51 d_{h 5, h 1}=\frac{2}{\tau_{\Theta 5}} \cdot \frac{1}{\beta 1_{h 1}} \cdot \frac{E_{\phi \phi}\left(\beta 1_{h 1}, r_{2}, r_{1}\right)}{P_{\phi}\left(\beta 1_{h 1}, r_{2}, r_{1}\right)} \cdot F_{3}^{\Theta}\left(\beta 1_{h 1}, \beta 5_{h 5}, \Theta_{1}, \Theta_{3}, \Theta_{3}, \tau_{\Theta 5}\right) . \\
& Q 52 c_{h 5, h 2}=-\frac{2}{\tau_{\Theta 5}} \cdot \frac{1}{\beta 2_{h 2}} \cdot \frac{E_{\phi}\left(\beta 2_{h 2}, r_{4}, r_{3}\right)}{P_{\phi}\left(\beta 2_{h 2}, r_{4}, r_{3}\right)} \cdot F_{3}^{\Theta}\left(\beta 2_{h 2}, \beta 5_{h 5}, \Theta_{1}, \Theta_{3}, \Theta_{3}, \tau_{\Theta 5}\right) . \\
& Q 56 c_{n 5, h 6}=-\frac{2}{\tau_{r 5}} \cdot \begin{cases}F_{3}^{r}\left(\lambda 5_{n 5}, r_{2}, r_{3}\right) & \text { for } h 6=0 \\
(-1)^{h 6} \cdot F_{5}^{r}\left(\beta 6_{h 6}, \lambda 5_{n 5}, r_{2}, r_{3}\right) & \text { for } h 6 \neq 0\end{cases} \\
& Q 56 d_{n 5, h 6}=-\frac{2}{\tau_{r 5}} \cdot \frac{r_{3}}{r_{2}} \cdot \begin{cases}F_{4}^{r}\left(\lambda 5_{n 5}, r_{2}, r_{3}\right) & \text { for } h 6=0 \\
(-1)^{h 6} \cdot F_{6}^{r}\left(\beta 6_{h 6}, \lambda 5_{n 5}, r_{2}, r_{3}\right) & \text { for } h 6 \neq 0\end{cases} \\
& Q 56 e_{n 5, n 6}=-\frac{2}{\tau_{r 5}} \cdot \frac{1}{\lambda 6_{n 6}} \cdot \operatorname{coth}\left(\lambda 6_{n 6} \cdot \tau_{\Theta 6}\right) \cdot F_{1}^{r}\left(\lambda 6_{n 6}, \lambda 5_{n 5}, r_{2}, r_{3}\right) \text { ， } \\
& Q 56 f_{n 5, n 6}=\frac{2}{\tau_{r} 5} \cdot \frac{1}{\lambda 6_{n 6}} \cdot \operatorname{csch}\left(\lambda 6_{n 6} \cdot \tau_{\Theta 6}\right) \cdot F_{1}^{r}\left(\lambda 6_{n 6}, \lambda 5_{n 5}, r_{2}, r_{3}\right) \\
& Q 57 c_{n 5, h 7}=-\frac{2}{\tau_{r 5}} \cdot \begin{cases}F_{3}^{r}\left(\lambda 5_{n 5}, r_{2}, r_{3}\right) & \text { for } h 7=0 \\
F_{5}^{r}\left(\beta 7_{h 7}, \lambda 5_{n 5}, r_{2}, r_{3}\right) & \text { for } h 7 \neq 0\end{cases} \\
& Q 57 d_{n 5, h 7}=-\frac{2}{\tau_{r 5}} \cdot \frac{r_{3}}{r_{2}} \cdot \begin{cases}F_{4}^{r}\left(\lambda 5_{n 5}, r_{2}, r_{3}\right) & \text { for } h 7=0 \\
F_{6}^{r}\left(\beta 7_{h 7}, \lambda 5_{n 5}, r_{2}, r_{3}\right) & \text { for } h 7 \neq 0\end{cases} \\
& Q 57 e_{n 5, n 7}=-\frac{2}{\tau_{r 5}} \cdot \frac{1}{\lambda 7_{n 7}} \cdot \operatorname{csch}\left(\lambda 7_{n 7} \cdot \tau_{\Theta 7}\right) \cdot F_{1}^{r}\left(\lambda 7_{n 7}, \lambda 5_{n 5}, r_{2}, r_{3}\right) \text {, } \\
& Q 57 f_{n 5, n 7}=\frac{2}{\tau_{r 5}} \cdot \frac{1}{\lambda 7_{n 7}} \cdot \operatorname{coth}\left(\lambda 7_{n 7} \cdot \tau_{\Theta 7}\right) \cdot F_{1}^{r}\left(\lambda 7_{n 7}, \lambda 5_{n 5}, r_{2}, r_{3}\right) \\
& E S 56_{n 5}=-\mu_{6} \cdot \frac{1}{2 \cdot \tau_{r 5}} \cdot \frac{1}{r_{2}} \cdot J_{z 6} \cdot F_{2}^{r}\left(\lambda 5_{n 5}, r_{2}, r_{3}\right) \\
& E S 57_{n 5}=-\mu_{7} \cdot \frac{1}{2 \cdot \tau_{r 5}} \cdot \frac{1}{r_{2}} \cdot J_{z 7} \cdot F_{2}^{r}\left(\lambda 5_{n 5}, r_{2}, r_{3}\right) \text {. }
\end{aligned}
$$


for Region 6

$$
\begin{aligned}
& Q 61 d_{h 6, h 1}=\frac{1}{\tau_{\Theta 6}} \cdot \frac{1}{\beta 1_{h 1}} \cdot \frac{E_{\phi}\left(\beta 1_{h 1}, r_{2}, r_{1}\right)}{P_{\phi}\left(\beta 1_{h 1}, r_{2}, r_{1}\right)} \cdot \begin{cases}F_{1}^{\Theta}\left(\beta 1_{h 1}, \Theta_{1}, \Theta_{2}, \tau_{\Theta 6}\right) & \text { for } h 6=0 \\
2 \cdot F_{2}^{\Theta}\left(\beta 6_{h 6}, \beta 1_{h 1}, \Theta_{2}, \Theta_{1}, \Theta_{2}, \tau_{\Theta 6}\right) & \text { for } h 6 \neq 0\end{cases} \\
& Q 62 c_{h 6, h 1}=-\frac{1}{\tau_{\Theta 6}} \cdot \frac{1}{\beta 2 h 2} \cdot \frac{E_{\phi}\left(\beta 2_{h 2}, r_{4}, r_{3}\right)}{P_{\phi}\left(\beta 2_{h 2}, r_{4}, r_{3}\right)} \cdot \begin{cases}F_{1}^{\Theta}\left(\beta 2_{h 2}, \Theta_{1}, \Theta_{2}, \tau_{\Theta 6}\right) & \text { for } h 6=0 \\
2 \cdot F_{2}^{\Theta}\left(\beta 6_{h 6}, \beta 2_{h 2}, \Theta_{2}, \Theta_{1}, \Theta_{2}, \tau_{\Theta 6}\right) & \text { for } h 6 \neq 0\end{cases} \\
& Q 63 c_{n 6, h 3}=-\frac{2 \cdot \beta 3_{h 3}}{\tau_{r 6}} \cdot \frac{\mu_{6}}{\mu_{3}} \cdot(-1)^{h 3} \cdot F_{5}^{r}\left(\beta 3_{h 3}, \lambda 6_{n 6}, r_{2}, r_{3}\right) \text {, } \\
& Q 63 d_{n 6, h 3}=-\frac{2 \cdot \beta 3_{h 3}}{\tau_{r 6}} \cdot \frac{\mu_{6}}{\mu_{3}} \cdot \frac{r_{3}}{r_{2}} \cdot(-1)^{h 3} \cdot F_{6}^{r}\left(\beta 3_{h 3}, \lambda 6_{n 6}, r_{2}, r_{3}\right) \text {, } \\
& Q 63 f_{n 6, n 3}=-\frac{2 \cdot \lambda 3_{n 3}}{\tau_{r 6}} \cdot \frac{\mu_{6}}{\mu_{3}} \cdot \operatorname{coth}\left(\lambda 3_{n 3} \cdot \tau_{\Theta 3}\right) \cdot F_{1}^{r}\left(\lambda 3_{n 3}, \lambda 6_{n 6}, r_{2}, r_{3}\right) \text {, } \\
& Q 65 c_{n 6, h 5}=-\frac{2 \cdot \beta 5_{h 5}}{\tau_{r 6}} \cdot \frac{\mu_{6}}{\mu_{5}} \cdot F_{5}^{\Theta}\left(\beta 5_{h 5}, \lambda 6_{n 6}, r_{2}, r_{3}\right), \\
& Q 65 d_{n 6, h 5}=-\frac{2 \cdot \beta 5_{h 5}}{\tau_{r 6}} \cdot \frac{\mu_{6}}{\mu_{5}} \cdot \frac{r_{3}}{r_{2}} \cdot F_{6}^{\Theta}\left(\beta 5_{h 5}, \lambda 6_{n 6}, r_{2}, r_{3}\right) \text { ， } \\
& Q 65 e_{n 6, n 5}=\frac{2 \cdot \lambda 5_{n 5}}{\tau_{r 6}} \cdot \frac{\mu_{6}}{\mu_{5}} \cdot \operatorname{coth}\left(\lambda 5_{n 5} \cdot \tau_{\Theta 5}\right) \cdot F_{1}^{r}\left(\lambda 5_{n 5}, \lambda 6_{n 6}, r_{2}, r_{3}\right) \text {, } \\
& Q 65 f_{n 6, n 5}=-\frac{2 \cdot \lambda 5_{n 5}}{\tau_{r 6}} \cdot \frac{\mu_{6}}{\mu_{5}} \cdot \operatorname{csch}\left(\lambda 5_{n 5} \cdot \tau_{\Theta 5}\right) \cdot F_{1}^{r}\left(\lambda 5_{n 5}, \lambda 6_{n 6}, r_{2}, r_{3}\right), \\
& E S 61_{0}=\frac{1}{4} \cdot \mu_{6} \cdot J_{z 6} \cdot r_{2} \\
& E S 62_{0}=\frac{1}{4} \cdot \mu_{6} \cdot J_{z 6} \cdot r_{3}
\end{aligned}
$$

for Region 7

$$
\begin{gathered}
Q 71 d_{h 7, h 1}=\frac{1}{\tau_{\Theta 7}} \cdot \frac{1}{\beta 1_{h 1}} \cdot \frac{E_{\phi}\left(\beta 1_{h 1}, r_{2}, r_{1}\right)}{P_{\phi}\left(\beta 1_{h 1}, r_{2}, r_{1}\right)} \cdot \begin{cases}F_{1}^{\Theta}\left(\beta 1_{h 1}, \Theta_{1}, \Theta_{4}, \tau_{\Theta 7}\right) & \text { for } h 7=0 \\
2 \cdot F_{2}^{\Theta}\left(\beta 7_{h 7}, \beta 1_{h 1}, \Theta_{4}, \Theta_{1}, \Theta_{4}, \tau_{\Theta 7}\right) & \text { for } h 7 \neq 0\end{cases} \\
Q 72 c_{h 7, h 2}=-\frac{1}{\tau_{\Theta 7}} \cdot \frac{1}{\beta 2_{h 2}} \cdot \frac{E_{\phi}\left(\beta 2_{h 2}, r_{4}, r_{3}\right)}{P_{\phi}\left(\beta 2_{h 2}, r_{4}, r_{3}\right)} \cdot \begin{cases}F_{1}^{\Theta}\left(\beta 2_{h 2}, \Theta_{1}, \Theta_{4}, \tau_{\Theta 7}\right) & \text { for } h 7=0 \\
2 \cdot F_{2}^{\Theta}\left(\beta 7_{h 7}, \beta 2_{h 2}, \Theta_{4}, \Theta_{1}, \Theta_{4}, \tau_{\Theta 7}\right) & \text { for } h 7 \neq 0\end{cases} \\
Q 74 c_{n 7, h 4}=-\frac{2 \cdot \beta 4_{h 4}}{\tau_{r 7}} \cdot \frac{\mu_{7}}{\mu_{4}} \cdot F_{5}^{r}\left(\beta 4_{h 4}, \lambda 7_{n 7}, r_{2}, r_{3}\right), \\
Q 74 d_{n 7, h 4}=-\frac{2 \cdot \beta 4_{h 4}}{\tau_{r 7}} \cdot \frac{\mu_{7}}{\mu_{4}} \cdot \frac{r_{3}}{r_{2}} \cdot F_{6}^{r}\left(\beta 4_{h 4}, \lambda 7_{n 7}, r_{2}, r_{3}\right), \\
Q 74 e_{n 7, n 4}=\frac{2 \cdot \lambda 4_{n 4}}{\tau_{r 7}} \cdot \frac{\mu_{7}}{\mu_{4}} \cdot \operatorname{coth}\left(\lambda 4_{n 4} \cdot \tau_{\Theta 4}\right) \cdot F_{1}^{r}\left(\lambda 4_{n 4}, \lambda 7_{n 7,}, r_{2}, r_{3}\right) . \\
Q 75 c_{n 7, h 5}=-\frac{2 \cdot \beta 5_{h 5}}{\tau_{r 7}} \cdot \frac{\mu_{7}}{\mu_{5}} \cdot(-1)^{h 5} \cdot F_{5}^{r}\left(\beta 5_{h 5}, \lambda 7_{n 7}, r_{2}, r_{3}\right), \\
Q 75 d_{n 7, h 5}=-\frac{2 \cdot \beta 5_{h 5}}{\tau_{r 7}} \cdot \frac{\mu_{7}}{\mu_{5}} \cdot \frac{r_{3}}{r_{2}} \cdot(-1)^{h 5} \cdot F_{6}^{r}\left(\beta 5_{h 5}, \lambda 7_{n 7}, r_{2}, r_{3}\right), \\
Q 75 e_{n 7, n 5}=\frac{2 \cdot \lambda 5_{n 5}}{\tau_{r 7}} \cdot \frac{\mu_{7}}{\mu_{5}} \cdot \operatorname{csch}\left(\lambda 5_{n 5} \cdot \tau_{\Theta 5}\right) \cdot F_{1}^{\Theta}\left(\lambda 5_{n 5}, \lambda 7_{\left.n 7, r_{2}, r_{3}\right),}\right.
\end{gathered}
$$




$$
\begin{gathered}
Q 75 f_{n 7, n 5}=-\frac{2 \cdot \lambda 5_{n 5}}{\tau_{r 7}} \cdot \frac{\mu_{7}}{\mu_{5}} \cdot \operatorname{coth}\left(\lambda 5_{n 5} \cdot \tau_{\Theta 5}\right) \cdot F_{1}^{\Theta}\left(\lambda 5_{n 5}, \lambda 7_{\left.n 7, r_{2}, r_{3}\right),}\right. \\
E S 71_{0}=\frac{1}{4} \cdot \mu_{7} \cdot J_{z 7} \cdot r_{2} \\
E S 72_{0}=\frac{1}{4} \cdot \mu_{7} \cdot J_{z 7} \cdot r_{3} .
\end{gathered}
$$

\section{References}

1. Dubas, F.; Boughrara, K. New scientific contribution on the 2-D subdomain technique in Cartesian coordinates: Taking into account of iron parts. Math. Comput. Appl. 2017, 22, doi: 10.3390/mca22010017.

2. Lehmann, T. Méthode graphique pour déterminer le trajet des lignes de force dans l'air. Revue d'Électricité: La Lumière Électrique 1909, 43-45, 103-110; 137-142; 163-168.

3. Jin, J. The Finite Element Method in Electromagnetic, 2nd ed.; John Wiley \& Sons, Inc.: New York, NY, USA, 2002.

4. Smith, G.D. Numerical Solution of Partial Differential Equations: Finite Difference Methods, 3rd ed.; Clarendon Press: Oxford, UK, 1985.

5. Wrobel, L.C.; Aliabadi, M.H. The Boundary Element Method; John Wiley \& Sons, Inc.: New York, NY, USA, 2002.

6. Kron, G. Equivalent circuits of electric machinery; John Wiley \& Sons, Inc.: New York, Chapman \& Hall, LTD., London, 1951.

7. Holman, J.P. Heat Transfer, 6th ed.; McGraw-Hill Book Compagny: New York, NY, USA, 1986.

8. Roters, H.C. Electromagnetic Devices; John Wiley \& Sons, Inc.: New York, NY, USA, 1941.

9. Driscoll, T.A.; Trefethen, L.N. Schwarz-Christoffel Mapping; Cambridge University Press: Cambridge, UK, 2002.

10. Hague, B. Electromagnetic Problems in Electrical Engineering; Oxford University Press: London, UK, 1929.

11. Sylvester, P. Modern Electromagnetic Fields; Prentice-Hall: London, UK, 1968.

12. Stoll, R.L. The Analysis of Eddy Currents; Clarendon Press: Oxford, UK, 1974.

13. Binns, K.J.; Lawrenson, P.J.; Trowbridge, C.W. The Analytical and Numerical Solution of Electric and Magnetic Fields; John Wiley \& Sons, Inc.: New York, NY, USA, 1992.

14. Melcher, J.R. Continuum Electromechanics; MIT Press: Cambridge, MA, USA, 1981.

15. Farlow, S.J. Partial Differential Equations for Scientists and Engineers; Dover, Inc.: New York, NY, USA, 1993.

16. Dubas, F.; Espanet, C. Analytical solution of the magnetic field in permanent-magnet motors taking into account slotting effect: No-load vector potential and flux density calculation. IEEE Trans. Magn. 2009, 45, 2097-2109.

17. Tiegna, H.; Amara, Y.; Barakat, G. Overview of analytical models of permanent magnet electrical machines for analysis and design purposes. Math. Comput. Simul. 2013, 90, 162-177.

18. Devillers, E.; Le Besnerais, J.; Lubin, T; Hecquet, M.; Lecointe, J.P. A review of subdomain modeling techniques in electrical machines: Performances and applications. In Proceedings of the XXII ${ }^{\text {th }}$ International Conference on Electrical Machines (ICEM), Lausanne, Switzerland, 4-7 September 2016.

19. Kumar, P.; Bauer, P. Improved analytical model of a permanent-magnet brushless DC motor. IEEE Trans. Magn. 2008, 44, 2299-2309.

20. Dalal, A.; Kumar, P. Analytical model for permanent magnet motor with slotting effect, armature reaction, and ferromagnetic material property. IEEE Trans. Magn. 2015, 51, doi:10.1109/TMAG.2015.2459036.

21. Boules, N. Two-dimensional field analysis of cylindrical machines with permanent magnet excitation. IEEE Trans. Ind. Appl. 1984, IA-20, 1267-1277.

22. Berkani, M.S.; Sough, M.L.; Giurgea, S.; Dubas, F.; Boualem, B.; Espanet, C. A simple analytical approach to model saturation in surface mounted permanent magnet synchronous motors. In Proceedings of the 2015 IEEE Energy Conversion Congress and Exposition (ECCE), Montreal, QC, Canada, 20-24 September 2015.

23. Mishkin, E. Theory of the squirrel-cage induction machine derived directly from Maxwell's field equations. Q. J. Mech. Appl. Math. 1954, 7, 472-487.

24. Panaitescu, A.; Panaitescu, I. A field model for induction machines. In Proceedings of the International Conference on Electrical Machines (ICEM), Vigo, Spain, 10-12 September 1996.

25. Madescu, G.; Boldea, I.; Miller, T.J.E. An analytical iterative model (AIM) for induction motor design. In Proceedings of the Conference Record of the 1996 IEEE Industry Applications Conference Thirty-First IAS Annual Meeting, San Diego, CA, USA, 6-10 October 1996. 
26. Sprangers, R.L.J.; Motoasca, T.E.; Lomonova, E.A. Extended anisotropic layer theory for electrical machines. IEEE Trans. Magn. 2013, 49, 2217-2220.

27. Sprangers, R.L.J.; Paulides, J.J.H.; Gysen, B.L.J.; Lomonova, E.A. Magnetic saturation in semi-analytical harmonic modeling for electric machine analysis. IEEE Trans. Magn. 2016, 52, doi:10.1109/TMAG.2015.2480708.

28. Sprangers, R.L.J.; Paulides, J.J.H.; Gysen, B.L.J.; Waarma, J.; Lomonova, E.A. Semi-analytical framework for synchronous reluctance motor analysis including finite soft-magnetic material permeability. IEEE Trans. Magn. 2015, 51, doi:10.1109/TMAG.2015.2442419.

29. Djelloul, K.Z.; Boughrara, K.; Ibtiouen, R.; Dubas, F. Nonlinear analytical calculation of magnetic field and torque of switched reluctance machines. In Proceedings of the CISTEM, Marrakech-Benguérir, Maroc, 26-28 October 2016.

30. Djelloul, K.Z.; Boughrara, K.; Dubas, F.; Ibtiouen, R. Nonlinear analytical prediction of magnetic field and electromagnétic performances in switched reluctance machines. IEEE Trans. Magn. 2017, 53, doi:10.1109/TMAG.2017.2679686.

31. Theodoulidis, T.P. Model of ferrite-cored probes for eddy current nondestructive evaluation. J. Appl. Phys. 2003, 93, 3071-3078.

32. Theodoulidis, T.P.; Bowler, J. Eddy-current interaction of a long coil with a slot in a conductive plate. IEEE Trans. Magn. 2005, 41, 1238-1247.

33. Roubache, L.; Boughrara, K.; Dubas, F.; Ibtiouen, R. Semi-Analytical modeling of spoke-type permanent-magnet machines considering the iron core relative permeability: Subdomain technique and Taylor polynomial. Progress In Electromagnetics Research B 2017, Accepted paper.

34. Roubache, L.; Boughrara, K.; Dubas, F.; Ibtiouen, R. Semi-analytical modeling of spoke-type permanent-magnet machines considering nonlinear magnetic saturation: Subdomain Technique and Taylor polynomial. Mathematics and Computers in Simulation 2017, Under review.

35. Abdel-Razek, A.A.; Coulomb, J.L.; Feliachi, M.; Sabonnadière, J.C. The calculation of electromagnetic torque in saturated electric machines within combined numerical and analytical solutions of the field equations. IEEE Trans. Magn. 1981, 17, 3250-3252.

36. Liu, Z.J.; Bi, C.; Tan, H.C.; Low, T.S. A combined numerical and analytical approach for magnetic field analysis of permanent magnet machines. IEEE Trans. Magn. 1995, 31, 1372-1375.

37. Mirzayee, M.; Mehrjerdi, H.; Tsurkerman, I. Analysis of a high-speed solid rotor induction motor using coupled analytical method and reluctance networks. In Proceedings of the 2005 IEEE/ACES International Conference on Wireless Communications and Applied Computational Electromagnetics (ACES), Honolulu, HI, USA, 3-7 April 2005.

38. Hemeida, A.; Sergeant, P. Analytical modeling of surface PMSM using a combined solution of Maxwell's equations and magnetic equivalent circuit. IEEE Trans. Magn. 2014, 50, doi:10.1109/TMAG.2014.2330801.

39. Pluk, K.J.W.; Jansen, J.W.; Lomonova, E.A. 3-D hybrid analytical modeling: 3-D Fourier modeling combined with mesh-based 3-D magnetic equivalent circuits. IEEE Trans. Magn. 2015, 51, doi:10.1109/TMAG.2015.2455951.

40. Flux2D. General Operating Instructions; Version 10.2.1; Cedrat S.A. Electrical Engineering: Grenoble, France, 2008.

41. Lee, S.W.; Jones, W.; Campbell, J. Convergence of numerical solution of iris-type discontinuity problems. IEEE Trans. Microw. Theory Tech. 1971, 19, 528-536.

42. Mittra, R.; Itoh, T.; Li, T-S. Analytical and numerical studies of the relative convergence phenomenon arising in the solution of an integral equation by the moment method. IEEE Trans. Microw. Theory Tech. 1972, 20, 96-104.

43. Rahideh, A.; Korakianitis, T. Analytical calculation of open-circuit magnetic field distribution of slotless brushless PM machines. Electrical Power and Energy Systems 2012, 44, 99-114.

44. Dubas, F.; Rahideh, A. 2-D analytical PM eddy-current loss calculations in slotless PMSM equipped with surface-inset magnets. IEEE Trans. Magn. 2014, 50, doi:10.1109/TMAG.2013.2285525.

45. Gysen, B.L.J.; Meessen, K.J.; Paulides, J.J.H.; Lomonova, E.A. General formulation of the electromagnetic field distribution in machines and devices using Fourier analysis. IEEE Trans. Magn. 2010, 46, 39-52. 